\title{
The Closest Elastic Tensor of Arbitrary Symmetry to an Elasticity Tensor of Lower Symmetry
}

\author{
Maher Moakher • Andrew N. Norris
}

Received: 21 February 2006 / Accepted: 22 August 2006 /

Published online: 5 October 2006

(C) Springer Science + Business Media B.V. 2006

\begin{abstract}
The closest tensors of higher symmetry classes are derived in explicit form for a given elasticity tensor of arbitrary symmetry. The mathematical problem is to minimize the elastic length or distance between the given tensor and the closest elasticity tensor of the specified symmetry. Solutions are presented for three distance functions, with particular attention to the Riemannian and log-Euclidean distances. These yield solutions that are invariant under inversion, i.e., the same whether elastic stiffness or compliance are considered. The Frobenius distance function, which corresponds to common notions of Euclidean length, is not invariant although it is simple to apply using projection operators. A complete description of the Euclidean projection method is presented. The three metrics are considered at a level of detail far greater than heretofore, as we develop the general framework to best fit a given set of moduli onto higher elastic symmetries. The procedures for finding the closest elasticity tensor are illustrated by application to a set of 21 moduli with no underlying symmetry.
\end{abstract}

Key words elastic symmetry • anisotropy $\cdot$ closest moduli $\cdot$ Reimannian metric • log-Euclidean

Mathematics Subject Classifications (2000) 73 C30 • 74B05 15 A $48 \cdot 15$ A69

M. Moakher

Laboratory for Mathematical and Numerical Modeling in Engineering Science, National Engineering School at Tunis, ENIT-LAMSIN, B.P. 37, 1002 Tunis Belvédère, Tunisia e-mail: maher.moakher@enit.rnu.tn

A. N. Norris $(\varangle)$

Department of Mechanical and Aerospace Engineering, Rutgers University, 98 Brett Road, Piscataway, NJ 08854-8058, USA

e-mail: norris@rutgers.edu 


\section{Introduction}

We address the question of finding the elastic moduli with a given material symmetry closest to an arbitrary set of elastic constants. There are several reasons for reducing a set of elastic constants in this way. One might desire to fit a data set to the $a$ priori known symmetry of the material. Alternatively, a seismic simulation might be best understood in terms of a model of the earth as a layered transversely isotropic medium, even though core samples indicate local anisotropy of a lower symmetry. More commonly, one might simply want to reduce the model complexity by decreasing the number of elastic parameters. In each case a distance function measuring the difference between sets of elastic moduli is necessary to define an appropriate closest set. The most natural metric is the Euclidean norm in which the "length" $\|\mathbb{C}\|$ of a set of moduli ${ }^{1} C_{i j k l}$ is $\|\mathbb{C}\|=\left(C_{i j k l} C_{i j k l}\right)^{1 / 2}$. The Euclidean distance function is, however, not invariant under inversion of the elasticity tensor - one obtains a different result using compliance as compared with stiffness. Alternative distance functions have been proposed which do not have this failing. In particular, the Riemannian distance function of Moakher [1] and the log-Euclidean metric of Arsigny et al. [2] are invariant under inversion, and as such are preferred general elastic norms.

The problem of simplifying elastic moduli by increasing the elastic symmetry was apparently first considered by Gazis et al. [3]. They provided a general framework for defining and deriving the Euclidean projection, i.e., the closest elasticity using the Euclidean norm. Several examples were given, including the closest isotropic material, which agrees with the isotropic approximant derived by Fedorov [4]. Fedorov obtained isotropic moduli using a different criterion: the isotropic material that best approximates the elastic wave velocities of the given moduli by minimizing the difference in the orientation averaged acoustical tensors. It may be shown [5] that the generalization of Fedorov's criterion to other symmetries is satisfied by the Euclidean projection of the stiffness tensor onto the elastic symmetry considered. The Euclidean projection is also equivalent to operating on the given elasticity tensor with the elements of the transformation group of the symmetry in question [6]. This approach has been used by François et al. [7] to find the closest moduli of trigonal and other symmetries for a set of ultrasonically measured stiffnesses. The equivalence of the projection and group transformation averaging will be discussed in Section 5. The Euclidean projection approach has received attention in the geophysical community from modelers interested in fitting rock data to particular elastic symmetries [8-11]. The work of Helbig [9] is particularly comprehensive. He considers the problem both in terms of the $6 \times 6$ matrix notation $[12,13]$ and in terms of 21-dimensional vectors representing the elastic moduli. The latter approach has been developed further by Browaeys and Chevrot [14] who describe the projection operators for different elastic symmetries in the 21-dimensional viewpoint. Dellinger [15] presents an algorithm for finding the closest transversely isotropic medium by searching over all orientations of the symmetry axis. Dellinger et al. [16] proposed a distinct approach to the problem of finding closest elasticity, based on the idea of generalized rotation of the 21-dimensional elasticity vector.

1 Lower case Latin suffices take on the values 1,2, and 3, and the summation convention on repeated indices is assumed.

Springer 
Although the Euclidean projection is commonly used in applications, it suffers from the fundamental drawback alluded to earlier, i.e., it is not invariant under inversion of the elasticity tensor. The projection found using the elastic stiffness is different from that obtained using the compliance tensor. This fundamental inconsistency arises from the dual physical properties of the elasticity tensor/matrix and its inverse. The projection of one is clearly not the same as the inverse of the projection of the other, although both projections by definition possess the same elastic symmetry. While there are circumstances in which the Euclidean projection is preferred, e.g., for the generalized Fedorov problem of finding the best acoustical approximant of a given symmetry [5], there is a clear need for a consistent technique to define a "closest" elastic material of a given symmetry. The solution to this quandary is to use an elastic distance function that is invariant under inversion. Several have been proposed of which we focus on two, the Riemannian distance function due to Moakher [1] and the log-Euclidean length of Arsigny et al. [2], described in Section 3. Moakher [1] describes how the Riemannian distance function gives a consistent method for averaging elasticity tensors. The only other application so far of these invariant distance functions to elasticity is by Norris [17] who discusses the closest isotropic elasticity.

The outline of the paper is as follows. The three distance functions are introduced in Section 3 after a brief review of notation in Section 2. Section 4 defines the different types of elastic symmetry using the algebraic tensor decomposition of Walpole [19]. Euclidean projection is presented in Section 5, with results for particular elastic symmetries summarized in Appendix A. Before discussing the closest tensors using the logarithmic norms, methods to evaluate the exponential and other functions of elasticity tensors are first described in Section 6. Section 7 discusses the closest tensors using the logarithmic norms, with particular attention given to isotropy and cubic symmetry as the target symmetries. Numerical examples are given in Section 8, and final conclusions in Section 9.

\section{Notation}

Elasticity tensors relate stress $\mathbf{T}$ and infinitesimal strain $\mathbf{E}$ linearly according to

$$
\mathbf{T}=\mathbb{C} \mathbf{E}, \quad \mathbf{E}=\mathbb{S} \mathbf{T},
$$

where $\mathbb{C}$ and $\mathbb{S}$ denote the fourth-order stiffness and compliance tensors, respectively. They satisfy $\mathbb{C}=\mathbb{S} \mathbb{C}=\mathbb{I}$, the identity. Although we are concerned primarily with fourth-order tensors in three-dimensional space, calculation and presentation are sometimes better performed using second-order tensors in sixdimensions. Accordingly we define $\mathcal{S}(d, r)$ as the space of symmetric tensors of order $r$ in $d$-dimensions. Elasticity tensors, denoted by $\mathbb{E}$ la $\subset \mathcal{S}(3,4)$, are positive definite, i.e., $\mathbb{A} \in \mathbb{E}$ la if $\langle\mathbf{B}, \mathbb{A} \mathbf{B}\rangle>0$ for all nonzero $\mathbf{B} \in \mathcal{S}(3,2)$. Components are defined relative to the basis triad $\left\{\mathbf{e}_{1}, \mathbf{e}_{2}, \mathbf{e}_{3}\right\}$; thus, $\mathbf{a}=a_{j} \mathbf{e}_{j}, \mathbf{A}=A_{i j} \mathbf{e}_{i} \otimes \mathbf{e}_{j}$, and $\mathbb{A}=$ $A_{i j k l} \mathbf{e}_{i} \otimes \mathbf{e}_{j} \otimes \mathbf{e}_{k} \otimes \mathbf{e}_{l}$, where the summation convention is assumed over 1, 2, 3 for lower case subscripts. Symmetry of second-order $(r=2)$ tensors implies $A_{i j}=A_{j i}$, while for $\mathbb{A} \in \mathcal{S}(3,4)$ the elements satisfy

$$
A_{i j k l}=A_{j i k l}=A_{i j l k}, \quad A_{i j k l}=A_{k l i j}
$$


The first pair of identities reflects the symmetry of the stress and the strain, while the last one is a consequence of the assumed existence of a strain energy function, and consequently elasticity tensors have at most 21 independent components.

Throughout the paper lower case Latin, upper case Latin and "ghostscript" indicate, respectively, three-dimensional vectors and tensors of order 2 and 4; e.g., vector $\mathbf{b}, \mathbf{A} \in \mathcal{S}(3,2), \mathbb{A} \in \mathcal{S}(3,4)$. The basis vectors are assumed orthonormal, $\mathbf{e}_{i} \cdot \mathbf{e}_{j}=\delta_{i j}$, so that products of tensors are defined by summation over pairs of indices: $(\mathbf{A B})_{i j}=A_{i k} B_{k j}$ and $(\mathbb{A} \mathbb{B})_{i j k l}=A_{i j p q} B_{p q k l}$. The inner product for tensors is defined as

$$
\langle u, v\rangle=\operatorname{tr}(u v),
$$

where $\operatorname{tr} \mathbf{A}=A_{i i}$, and for elasticity tensors, $\operatorname{tr} \mathbb{A}=A_{i j i j}$. The norm of a tensor is

$$
\|u\| \equiv\langle u, u\rangle^{1 / 2}
$$

We take advantage of the well known isomorphisms between $\mathcal{S}(3,2)$ and $\mathcal{S}(6,1)$, and between $\mathcal{S}(3,4)$ and $\mathcal{S}(6,2)$. Thus, fourth-order elasticity tensors in three dimensions are equivalent to second-order symmetric tensor of six dimensions [20], with properties $\mathbb{B} \mathbf{A} \leftrightarrow \widehat{\mathbf{B}} \hat{\mathbf{a}}, \mathbb{A} \mathbb{B} \leftrightarrow \widehat{\mathbf{A}} \widehat{\mathbf{B}}$, and $\langle\mathbb{A}, \mathbb{B}\rangle=\langle\widehat{\mathbf{A}}, \widehat{\mathbf{B}}\rangle$. Vectors and secondorder tensors in six dimension are distinguished by a hat, e.g., vector $\hat{\mathbf{a}}, \widehat{\mathbf{A}} \in \mathcal{S}(6,2)$. Components are defined relative to the orthonormal sextet $\left\{\hat{\mathbf{e}}_{I}, I=1,2, \ldots, 6\right\}$, $\hat{\mathbf{e}}_{I} \cdot \hat{\mathbf{e}}_{J}=\delta_{I J}$, by $\hat{\mathbf{a}}=a_{I} \hat{\mathbf{e}}_{I}, \widehat{\mathbf{A}}=\widehat{A}_{I J} \hat{\mathbf{e}}_{I} \otimes \hat{\mathbf{e}}_{J}$, with the summation convention over $1,2, \ldots, 6$ for capital subscripts. Also, $\operatorname{tr} \widehat{\mathbf{A}}=\widehat{A}_{I I}$. The connection between $\mathcal{S}(6,1)$ and $\mathcal{S}(3,2)$ is made concrete by relating the basis vectors:

$$
\begin{aligned}
& 6 \text { - vector dyadic } \\
& \begin{array}{lllll}
\hat{\mathbf{e}}_{1} & \hat{\mathbf{e}}_{4} & \leftrightarrow & \mathbf{e}_{1} \otimes \mathbf{e}_{1} & \frac{1}{\sqrt{2}}\left(\mathbf{e}_{2} \otimes \mathbf{e}_{3}+\mathbf{e}_{3} \otimes \mathbf{e}_{2}\right) \\
\hat{\mathbf{e}}_{2} & \hat{\mathbf{e}}_{5} & & \mathbf{e}_{2} \otimes \mathbf{e}_{2} & \frac{1}{\sqrt{2}}\left(\mathbf{e}_{3} \otimes \mathbf{e}_{1}+\mathbf{e}_{1} \otimes \mathbf{e}_{3}\right) \\
\hat{\mathbf{e}}_{3} & \hat{\mathbf{e}}_{6} & & \mathbf{e}_{3} \otimes \mathbf{e}_{3} & \frac{1}{\sqrt{2}}\left(\mathbf{e}_{1} \otimes \mathbf{e}_{2}+\mathbf{e}_{2} \otimes \mathbf{e}_{1}\right)
\end{array}
\end{aligned}
$$

This implies a unique $\widehat{\mathbf{A}} \in \mathcal{S}(6,2)$ for each $\mathbb{A} \in \mathcal{S}(3,4)$, and vice versa. Let $\mathbb{C} \in \mathbb{E}$ la be the tensor of elastic stiffness, usually defined by the Voigt notation: $C_{i j k l} \equiv c_{I J}$, where $I$ or $J=1,2,3,4,5,6$ correspond to $i j$ or $k l=11,22,33,23,13,12$, respectively, and $c_{I J}$ are the elastic moduli in the Voigt notation [21], i.e.,

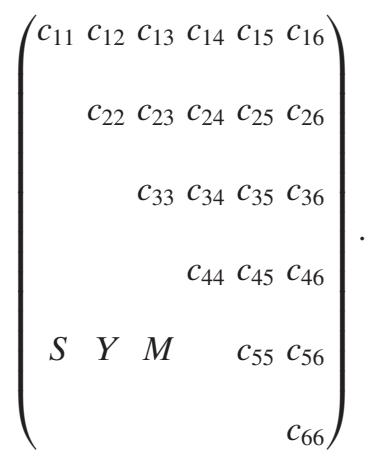


The isomorphism implies that the associated matrix $\widehat{\mathbf{C}} \in \mathcal{S}(6,2)$ is positive definite and has elements

$$
\widehat{\mathbf{C}} \equiv\left(\begin{array}{cccccc}
c_{11} & c_{12} & c_{13} & 2^{\frac{1}{2}} c_{14} & 2^{\frac{1}{2}} c_{15} & 2^{\frac{1}{2}} c_{16} \\
c_{12} & c_{22} & c_{23} & 2^{\frac{1}{2}} c_{24} & 2^{\frac{1}{2}} c_{25} & 2^{\frac{1}{2}} c_{26} \\
c_{13} & c_{23} & c_{33} & 2^{\frac{1}{2}} c_{34} & 2^{\frac{1}{2}} c_{35} & 2^{\frac{1}{2}} c_{36} \\
2^{\frac{1}{2}} c_{14} & 2^{\frac{1}{2}} c_{24} & 2^{\frac{1}{2}} c_{34} & 2 c_{44} & 2 c_{45} & 2 c_{46} \\
2^{\frac{1}{2}} c_{15} & 2^{\frac{1}{2}} c_{25} & 2^{\frac{1}{2}} c_{35} & 2 c_{45} & 2 c_{55} & 2 c_{56} \\
2^{\frac{1}{2}} c_{16} & 2^{\frac{1}{2}} c_{26} & 2^{\frac{1}{2}} c_{36} & 2 c_{46} & 2 c_{56} & 2 c_{66}
\end{array}\right) .
$$

The spectral decomposition of elasticity tensors can be expressed in both the fourth-order and second-order notations $[12,20]$,

$$
\mathbb{C}=\sum_{I=1}^{6} \Lambda_{I} \mathbf{N}_{I} \otimes \mathbf{N}_{I} \quad \leftrightarrow \quad \widehat{\mathbf{C}}=\sum_{I=1}^{6} \Lambda_{I} \hat{\mathbf{n}}_{I} \otimes \hat{\mathbf{n}}_{I},
$$

where $\Lambda_{I}$ and $\hat{\mathbf{n}}_{I}, I=1,2, \ldots, 6$ are the eigenvalues and eigenvectors of the matrix $\widehat{\mathbf{C}}$, $\mathbf{N}_{I}$ are the associated dyadics, with $\left\langle\hat{\mathbf{n}}_{I}, \hat{\mathbf{n}}_{J}\right\rangle=\left\langle\mathbf{N}_{I}, \mathbf{N}_{J}\right\rangle=\delta_{I J}$. Also, $\Lambda_{I}>0$ by virtue of the positive definite nature of the strain energy.

The elastic moduli $\mathbb{C}$ can also be expressed as a vector with 21 elements [14],

$$
\begin{aligned}
X= & \left(x_{1}, x_{2}, x_{3}, x_{4}, x_{5}, x_{6}, x_{7}, x_{8}, x_{9}, x_{10}, x_{11}, x_{12}, x_{13}, x_{14}, x_{15}, x_{16}, x_{17}, x_{18}, x_{19}, x_{20}, x_{21}\right)^{t} \\
= & \left(c_{11}, c_{22}, c_{33}, \sqrt{2} c_{23}, \sqrt{2} c_{13}, \sqrt{2} c_{12}, 2 c_{44}, 2 c_{55}, 2 c_{66}, 2 c_{14}, 2 c_{25}, 2 c_{36},\right. \\
& \left.2 c_{34}, 2 c_{15}, 2 c_{26}, 2 c_{24}, 2 c_{35}, 2 c_{16}, 2 \sqrt{2} c_{56}, 2 \sqrt{2} c_{46}, 2 \sqrt{2} c_{45}\right)^{t} \\
= & \left(\hat{c}_{11}, \hat{c}_{22}, \hat{c}_{33}, \sqrt{2} \hat{c}_{23}, \sqrt{2} \hat{c}_{13}, \sqrt{2} \hat{c}_{12}, \hat{c}_{44}, \hat{c}_{55}, \hat{c}_{66}, \sqrt{2} \hat{c}_{14}, \sqrt{2} \hat{c}_{25}, \sqrt{2} \hat{c}_{36},\right. \\
& \left.\sqrt{2} \hat{c}_{34}, \sqrt{2} \hat{c}_{15}, \sqrt{2} \hat{c}_{26}, \sqrt{2} \hat{c}_{24}, \sqrt{2} \hat{c}_{35}, \sqrt{2} \hat{c}_{16}, \sqrt{2} \hat{c}_{56}, \sqrt{2} \hat{c}_{46}, \sqrt{2} \hat{c}_{45}\right)^{t} .
\end{aligned}
$$

The $\sqrt{2}$ 's ensure that the inner product preserves the norm of the elastic moduli, whether it is a tensor, matrix or vector. Thus, the norm of an elasticity tensor can be expressed in various ways depending on how $\mathbb{C}$ is represented,

$$
\|\mathbb{C}\|^{2} \equiv\langle\mathbb{C}, \mathbb{C}\rangle=C_{i j k l} C_{i j k l}=\widehat{c}_{I J} \widehat{c}_{I J}=\Lambda_{I} \Lambda_{I}=\|X\|^{2}
$$




\section{Elastic Distance Functions}

We consider three metrics for Ela: the Euclidean or Frobenius metric $\mathrm{d}_{F}$, the logEuclidean norm $\mathrm{d}_{L}$ [2] and the Riemannian metric $\mathrm{d}_{R}$ [1]. They are defined for any pair of elasticity tensors as

$$
\begin{aligned}
& \mathrm{d}_{F}\left(\mathbb{C}_{1}, \mathbb{C}_{2}\right)=\left\|\mathbb{C}_{1}-\mathbb{C}_{2}\right\|, \\
& \mathrm{d}_{L}\left(\mathbb{C}_{1}, \mathbb{C}_{2}\right)=\left\|\log \left(\mathbb{C}_{1}\right)-\log \left(\mathbb{C}_{2}\right)\right\|, \\
& \mathrm{d}_{R}\left(\mathbb{C}_{1}, \mathbb{C}_{2}\right)=\left\|\log \left(\mathbb{C}_{1}^{-1 / 2} \mathbb{C}_{2} \mathbb{C}_{1}^{-1 / 2}\right)\right\| .
\end{aligned}
$$

The Riemannian distance $\mathrm{d}_{R}$ is related to the exponential map induced by the scalar product on the tangent space to $\mathbb{E}$ la at $\mathbb{C}[1,22]$. The log-Euclidean metric $\mathrm{d}_{L}$ can be motivated by the following definition of tensor multiplication [2] :

$$
\mathbb{C}_{1} \odot \mathbb{C}_{2} \equiv \exp \left(\log \left(\mathbb{C}_{1}\right)+\log \left(\mathbb{C}_{2}\right)\right) \text {. }
$$

The $\odot$ product preserves symmetry and positive definiteness, unlike normal multiplication. The three metrics have the usual attributes of a distance function $\mathrm{d}$ :

1. non-negative, $\mathrm{d}\left(\mathbb{C}_{1}, \mathbb{C}_{2}\right) \geq 0$ with equality iff $\mathbb{C}_{1}=\mathbb{C}_{2}$,

2. symmetric in the arguments, $\mathrm{d}\left(\mathbb{C}_{1}, \mathbb{C}_{2}\right)=\mathrm{d}\left(\mathbb{C}_{2}, \mathbb{C}_{1}\right)$,

3. invariant under a change of basis, $\mathrm{d}\left(\mathbb{C}_{1}^{\prime}, \mathbb{C}_{2}^{\prime}\right)=\mathrm{d}\left(\mathbb{C}_{1}, \mathbb{C}_{2}\right)$ for all proper orthogonal coordinate transformations $\left\{\mathbf{e}_{1}, \mathbf{e}_{2}, \mathbf{e}_{3}\right\} \rightarrow\left\{\mathbf{e}_{1}^{\prime}, \mathbf{e}_{2}^{\prime}, \mathbf{e}_{3}^{\prime}\right\}$, and

4. it satisfies the triangle inequality, $\mathrm{d}\left(\mathbb{C}_{1}, \mathbb{C}_{3}\right) \leq \mathrm{d}\left(\mathbb{C}_{1}, \mathbb{C}_{2}\right)+\mathrm{d}\left(\mathbb{C}_{2}, \mathbb{C}_{3}\right)$.

The Riemannian and log-Euclidean distances possess the additional properties that they are invariant under inversion and (positive) scalar multiplication

$$
\mathrm{d}_{L, R}\left(\mathbb{C}_{1}^{-1}, \mathbb{C}_{2}^{-1}\right)=\mathrm{d}_{L, R}\left(\mathbb{C}_{1}, \mathbb{C}_{2}\right), \text { and } \mathrm{d}_{L, R}(a \mathbb{C}, a \mathbb{C})=\mathrm{d}_{L, R}\left(\mathbb{C}_{1}, \mathbb{C}_{2}\right), a>0 .
$$

The following inequality between the logarithmic distance functions is a consequence of the metric increasing property of the exponential [23]

$$
\mathrm{d}_{L}\left(\mathbb{C}_{1}, \mathbb{C}_{2}\right) \leq \mathrm{d}_{R}\left(\mathbb{C}_{1}, \mathbb{C}_{2}\right)
$$

We also have (see [23] for the inequality)

$$
\begin{array}{ll}
\mathrm{d}_{L}\left(\mathbb{C}_{1}^{b}, \mathbb{C}_{2}^{b}\right)=|b| \mathrm{d}_{L}\left(\mathbb{C}_{1}, \mathbb{C}_{2}\right), & b \in \mathbb{R} \\
\mathrm{d}_{R}\left(\mathbb{C}_{1}^{b}, \mathbb{C}_{2}^{b}\right) \leq b \mathrm{~d}_{R}\left(\mathbb{C}_{1}, \mathbb{C}_{2}\right), & b \in[0,1],
\end{array}
$$

and the Riemannian distance is invariant under congruent transformations, i.e.,

$$
\mathrm{d}_{R}\left(\mathbb{T} \mathbb{C}_{1} \mathbb{T}^{t}, \mathbb{T} \mathbb{C}_{2} \mathbb{T}^{t}\right)=\mathrm{d}_{R}\left(\mathbb{C}_{1}, \mathbb{C}_{2}\right), \quad \forall \mathbb{T} \text { invertible. }
$$

Distance functions satisfying Eq. (13) are called bi-invariant, a property that makes the Riemannian and log-Euclidean distances consistent metrics for elasticity tensors. The Frobenius norm, not being invariant under inversion, gives different results depending on whether the stiffness or compliance is considered. Other metric functions may be considered. For instance, the Kullback-Leibler metric [1] is invariant under inversion and congruent transformation but does not satisfy the triangle inequality, which we take here as prerequisite for consideration as a distance function. 
Each distance function has a geometrical interpretation. For instance, the midpoint between $\mathbb{C}_{1}$ and $\mathbb{C}_{2}$ is defined as the unique $\mathbb{C}_{3}$ such that $\mathrm{d}\left(\mathbb{C}_{1}, \mathbb{C}_{3}\right)=$ $\mathrm{d}\left(\mathbb{C}_{3}, \mathbb{C}_{2}\right)=\frac{1}{2} \mathrm{~d}\left(\mathbb{C}_{1}, \mathbb{C}_{2}\right)$. Using $\mathrm{d}_{F}$, the midpoint is the vector halfway between the 21-vectors $X_{1}$ and $X_{2}$ as defined by Eq. (9), i.e., $\mathbb{C}_{3}=\frac{1}{2}\left(\mathbb{C}_{1}+\mathbb{C}_{2}\right)$. The midpoint using $\mathrm{d}_{L}$ is $\mathbb{C}_{3}=\left(\mathbb{C}_{1} \odot \mathbb{C}_{2}\right)^{1 / 2}$. The midpoint with $\mathrm{d}_{R}$ is [24] $\mathbb{C}_{3}=\mathbb{C}_{1}\left(\mathbb{C}_{1}^{-1} \mathbb{C}_{2}\right)^{1 / 2}$ which can be expressed in several other ways, see [1]. More generally, the midpoint is the value of the geodesic $\mathbb{C}(t)$ at $t=\frac{1}{2}$, where

$$
\mathbb{C}(t)= \begin{cases}(1-t) \mathbb{C}_{1}+t \mathbb{C}_{2}, & \text { Frobenius, } \\ \exp \left((1-t) \log \left(\mathbb{C}_{1}\right)+t \log \left(\mathbb{C}_{2}\right)\right), & \text { log-Euclidean, } \\ \mathbb{C}_{1} \exp \left(t \log \left(\mathbb{C}_{1}^{-1} \mathbb{C}_{2}\right)\right), & \text { Riemannian, }\end{cases}
$$

\section{Elasticity Tensors of the Different Symmetry Classes}

Elasticity tensors for the different symmetry classes are described in this section. The choice of representation of $\mathbb{E}$ la is important for discussing the closest approximants, particularly for the bi-invariant metrics. It should preferably be independent of the coordinate system, although at the same time, practical application is normally in terms of the Voigt notation, so the format should not deviate too far from this. We begin with a review of alternative representations for elastic moduli.

\subsection{Representation of Elasticity Tensors}

There are many representations of $\mathbb{E}$ la in addition to the standard Voigt notation of Eq. (6). The 21-dimensional vector format [14] is useful for some applications, including the Euclidean projection, as we show later. However, these two representations depend upon the coordinate system. Among the coordinate-free forms of Ela that can be identified in the literature we distinguish (a) spectral decomposition, (b) algebraic decomposition, (c) groups and reflection symmetries, (d) harmonic decomposition, (e) integrity bases.

The first, (a), spectral decomposition dates back to Kelvin [25]. The idea is simple: the elasticity tensor operates on the six-dimensional space of symmetric secondorder tensors, and therefore has a six-dimensional spectral form. The associated eigenvalues are called the Kelvin moduli $[12,13]$. Recent related developments are due to Rychlewski [12] and Mehrabadi and Cowin [13, 20], who independently rediscovered and extended Kelvin's approach, see also [26, 27]. The associated sixdimensional tensor representation will be used here for practical implementation. (b) tensor functions operating on $\mathbb{E}$ la can be greatly simplified using the irreducible tensor algebra proposed by Walpole [19], and independently by Kunin [28]. This procedure provides the most efficient means of representing elastic tensors of a given symmetry class, especially those of high symmetry. We adopt this method to develop most of the results here. (c) the group of rotations associated with elastic symmetry provides an irreducible representation [29]. There are various related ways of considering elasticity tensors in terms of rotational group properties of tensors, e.g., based on Cartan decomposition [30], complex vectors and tensors [31,32], and subgroups of $O(3)[33,34]$. These ideas are closely related to definitions of elastic symmetry in terms of a single symmetry element: reflection about a plane. The necessary algebra 
and the relationship to the more conventional crystallographic symmetry elements are described in detail by Cowin and Mehrabadi [35]. Comparison between the rotation-based approach and that using symmetry planes is provided by Chadwick et al. [18]. (d) Backus [36] proposed a representation of $\mathbb{E}$ la in terms of harmonic tensors. These are based on an isomorphism between the space of homogeneous harmonic polynomials of degree $q$ and the space of totally symmetric tensors of order $q$. There has been considerable interest in Backus's representation [6, 37-39]. For instance, Baerheim and Helbig [40] provide an orthonormal decomposition of Ela in terms of harmonic tensors. (e) elasticity decomposition via integrity bases has been studied considerably [41-45]. An integrity basis is a set of polynomials, each invariant under the group of symmetry transformations, such that any polynomial function invariant under the group is expressible as a polynomial in elements of the integrity basis. For instance, Tu [42] used an integrity basis to construct five hierarchies of orthonormal tensor bases which span the space of elastic constants of all crystal systems. Any elastic tensor of order four possessing certain symmetry may be decomposed into a sum of tensors of increasing symmetry. The resulting decomposition has considerable similarity to the decomposition generated in Section 5 using Walpole's irreducible elements with Euclidean projection. Among other methods for decomposing elasticity tensors, we mention the scheme of Elata and Rubin [46] who use a set of six vectors related to a regular icosahedron to form a basis for $\mathbb{E}$ la. This basis naturally splits a tensor into deviatoric and non-deviatoric parts.

We use Walpole's [19] algebraic representation as it provides a consistent and straightforward means to define projections of fourth-order tensors onto the given elastic symmetry. This representation is better for our purposes than the spectral decomposition, since it is independent of the elastic moduli (i.e., no distributors [13]) and depends only on the crystallographic orientation. It uses the notion of basis tensors, similar to but not the same as for a vector space, which makes it very suitable for Euclidean projection. We work mainly with fourth-order tensors directly, although the associated six-dimensional matrix notation is also provided. The latter is simpler for purposes of computation, e.g., many of the matrix operations are easily implemented using MATLAB. We begin with the highest elastic symmetry.

\subsection{Isotropic System}

A general isotropic fourth-order tensor is given by

$$
\mathbb{A}=a \mathbb{J}+b \mathbb{K},
$$

where $\mathbb{J}$ and $\mathbb{K}$ are the two linearly independent symmetric tensors defined by

$$
\mathbb{J}=\frac{1}{3} \mathbf{I} \otimes \mathbf{I}, \quad \mathbb{K}=\mathbb{I}-\mathbb{J} .
$$

The component forms follow from $I_{i j k l}=\frac{1}{2}\left(\delta_{i k} \delta_{j l}+\delta_{i l} \delta_{j k}\right)$ and $J_{i j k l}=\frac{1}{3} \delta_{i j} \delta_{k l}$. Note that the tensors $\mathbb{J}$ and $\mathbb{K}$ sum to the identity,

$$
\mathbb{I}=\mathbb{J}+\mathbb{K}
$$

and they satisfy the multiplication table

$$
\mathbb{J}^{2}=\mathbb{J}, \quad \mathbb{K}^{2}=\mathbb{K}, \quad \mathbb{J} \mathbb{K}=\mathbb{K} \mathbb{J}=\mathbb{O} .
$$


The Euclidean lengths are $\|\mathbb{J}\|=1,\|\mathbb{K}\|=\sqrt{5}$. Tensors $\mathbb{J}$ and $\mathbb{K}$ form, respectively, one and five-dimensional Kelvin subspaces [13]. An important result (see [3, 4]) is that the closest isotropic elasticity tensor in the Euclidean sense is

$$
\mathbb{C}_{\text {iso }}=3 \kappa \mathbb{J}+2 \mu \mathbb{K},
$$

where

$$
\begin{aligned}
9 \kappa & =3\langle\mathbb{C}, \mathbb{J}\rangle=\hat{c}_{11}+\hat{c}_{22}+\hat{c}_{33}+2 \hat{c}_{12}+2 \hat{c}_{13}+2 \hat{c}_{23}, \\
30 \mu & =3\langle\mathbb{C}, \mathbb{K}\rangle=2\left(\hat{c}_{11}+\hat{c}_{22}+\hat{c}_{33}-\hat{c}_{12}-\hat{c}_{23}-\hat{c}_{31}\right)+3\left(\hat{c}_{44}+\hat{c}_{55}+\hat{c}_{66}\right) .
\end{aligned}
$$

Gazis et al. [3] obtained Eq. (22) using methods similar to those we will generalize in Section 5. Fedorov actually found $\kappa$ and $\mu$ using an apparently different approach the minimization of the mean square difference in the acoustical or Christoffel matrices for the original and isotropic systems. However, it can be shown [5] that the generalization of Fedorov's approach to other symmetries is identical to the Euclidean minimization. The moduli (23) will be derived later in the context of the general theory for Euclidean projection.

\subsection{Cubic System}

Let $\mathbf{a}, \mathbf{b}$ and $\mathbf{c}$ be three mutually orthogonal unit vectors that describe the three crystallographic directions of a cubic medium. We introduce the second-order tensors

$$
\begin{aligned}
& \mathbf{U}=\frac{1}{\sqrt{2}}(\mathbf{a} \otimes \mathbf{c}+\mathbf{c} \otimes \mathbf{a}), \quad \mathbf{V}=\frac{1}{\sqrt{2}}(\mathbf{b} \otimes \mathbf{c}+\mathbf{c} \otimes \mathbf{b}), \quad \mathbf{W}=\frac{1}{\sqrt{2}}(\mathbf{a} \otimes \mathbf{b}+\mathbf{b} \otimes \mathbf{a}), \\
& \mathbf{X}=\frac{1}{\sqrt{2}}(\mathbf{c} \otimes \mathbf{c}-\mathbf{a} \otimes \mathbf{a}), \quad \mathbf{Y}=\frac{1}{\sqrt{2}}(\mathbf{b} \otimes \mathbf{b}-\mathbf{c} \otimes \mathbf{c}), \quad \mathbf{Z}=\frac{1}{\sqrt{2}}(\mathbf{a} \otimes \mathbf{a}-\mathbf{b} \otimes \mathbf{b}),
\end{aligned}
$$

and fourth-order tensors $\mathbb{L}$ and $\mathbb{M}$ defined by them,

$$
\mathbb{L}=\mathbf{U} \otimes \mathbf{U}+\mathbf{V} \otimes \mathbf{V}+\mathbf{W} \otimes \mathbf{W}, \quad \mathbb{M}=\frac{2}{3}(\mathbf{X} \otimes \mathbf{X}+\mathbf{Y} \otimes \mathbf{Y}+\mathbf{Z} \otimes \mathbf{Z}) .
$$

The tensors $\mathbb{J}, \mathbb{L}$ and $\mathbb{M}$ sum up to the identity tensor, and $\mathbb{L}$ and $\mathbb{M}$ partition $\mathbb{K}$, i.e.,

$$
\mathbb{I}=\mathbb{J}+\mathbb{L}+\mathbb{M}, \quad \mathbb{K}=\mathbb{L}+\mathbb{M} .
$$

The multiplication table for cubic tensors is

$$
\mathbb{J}^{2}=\mathbb{J}, \quad \mathbb{L}^{2}=\mathbb{L}, \quad \mathbb{M}^{2}=\mathbb{M}, \quad \mathbb{L} L=\mathbb{L} \mathbb{J}=\mathbb{J} \mathbb{M}=\mathbb{M} \mathbb{I}=\mathbb{L} \mathbb{M}=\mathbb{M L}=\mathbb{O},
$$

and the Euclidean lengths are $\|\mathbb{L}\|=\sqrt{3},\|\mathbb{M}\|=\sqrt{2}$.

A general symmetric fourth-order tensor for a cubic media is given by a linear combination of the three linearly independent tensors $\mathbb{J}, \mathbb{L}$ and $\mathbb{M}$

$$
\mathbb{A}=a \mathbb{J}+b \mathbb{L}+c \mathbb{M} .
$$

The tensors $\mathbb{I}, \mathbb{L}$ and $\mathbb{M}$ form, respectively, one, three and two-dimensional Kelvin subspaces [13]. 
4.4 Transversely Isotropic System

Assume that the unit vector c characterizes the preferred direction of a transversely isotropic medium. Let $\mathbf{P}$ and $\mathbf{Q}$ be the second-order tensors

$$
\mathbf{P}=\mathbf{c} \otimes \mathbf{c}, \quad \mathbf{Q}=\frac{1}{\sqrt{2}}(\mathbf{I}-\mathbf{c} \otimes \mathbf{c}),
$$

and define the six linearly independent fourth-order elementary tensors

$$
\begin{array}{rlrl}
\mathbb{E}_{1} & =\mathbf{P} \otimes \mathbf{P}, \quad \mathbb{E}_{2}=\mathbf{Q} \otimes \mathbf{Q}, & \mathbb{E}_{3}=\mathbf{P} \otimes \mathbf{Q}, \quad \mathbb{E}_{4}=\mathbf{Q} \otimes \mathbf{P}, \\
\mathbb{F} & =\mathbf{W} \otimes \mathbf{W}+\mathbf{Z} \otimes \mathbf{Z}, & \mathbb{G} & =\mathbf{U} \otimes \mathbf{U}+\mathbf{V} \otimes \mathbf{V} .
\end{array}
$$

Both $\mathbb{E}_{1}$ and $\mathbb{E}_{2}$ are symmetric but the tensors $\mathbb{E}_{3}$ and $\mathbb{E}_{4}$ are not, although they are the transpose of one another and so their sum is a symmetric tensor. These asymmetric tensors are introduced because the set $\left\{\mathbb{E}_{1}, \mathbb{E}_{2}, \mathbb{E}_{3}+\mathbb{E}_{4}\right\}$ is not closed under tensor multiplication. The multiplication table for the six elementary tensors is

\begin{tabular}{c|cccccc} 
& $\mathbb{E}_{1}$ & $\mathbb{E}_{2}$ & $\mathbb{E}_{3}$ & $\mathbb{E}_{4}$ & $\mathbb{F}$ & $\mathbb{G}$ \\
\hline $\mathbb{E}_{1}$ & $\mathbb{E}_{1}$ & $\mathbb{O}$ & $\mathbb{E}_{3}$ & $\mathbb{O}$ & $\mathbb{O}$ & $\mathbb{O}$ \\
$\mathbb{E}_{2}$ & $\mathbb{O}$ & $\mathbb{E}_{2}$ & $\mathbb{O}$ & $\mathbb{E}_{4}$ & $\mathbb{O}$ & $\mathbb{O}$ \\
$\mathbb{E}_{3}$ & $\mathbb{O}$ & $\mathbb{E}_{3}$ & $\mathbb{O}$ & $\mathbb{E}_{1}$ & $\mathbb{O}$ & $\mathbb{O}$ \\
$\mathbb{E}_{4}$ & $\mathbb{E}_{4}$ & $\mathbb{O}$ & $\mathbb{E}_{2}$ & $\mathbb{O}$ & $\mathbb{O}$ & $\mathbb{O}$ \\
$\mathbb{F}$ & $\mathbb{O}$ & $\mathbb{O}$ & $\mathbb{O}$ & $\mathbb{O}$ & $\mathbb{F}$ & $\mathbb{O}$ \\
$\mathbb{G}$ & $\mathbb{O}$ & $\mathbb{O}$ & $\mathbb{O}$ & $\mathbb{O}$ & $\mathbb{O}$ & $\mathbb{G}$
\end{tabular}

The tensors $\mathbb{E}_{1}, \mathbb{E}_{2}, \mathbb{F}$ and $\mathbb{G}$ sum up to the identity,

$$
\mathbb{I}=\mathbb{E}_{1}+\mathbb{E}_{2}+\mathbb{F}+\mathbb{G} \text {. }
$$

The Euclidean lengths are $\left\|\mathbb{E}_{1}\right\|=\left\|\mathbb{E}_{2}\right\|=1,\left\|\mathbb{E}_{3}+\mathbb{E}_{4}\right\|=\|\mathbb{F}\|=\|\mathbb{G}\|=\sqrt{2}$.

A general symmetric fourth-order tensor of transversely isotropic symmetry is given by the five-parameter linear combination

$$
\mathbb{A}=a \mathbb{E}_{1}+b \mathbb{E}_{2}+c\left(\mathbb{E}_{3}+\mathbb{E}_{4}\right)+f \mathbb{F}+g \mathbb{G} .
$$

Note that the base tensors do not correspond to the Kelvin modes, although some can be identified as such, e.g., $\mathbb{G}$ is a two-dimensional Kelvin subspace [13]. The three bases tensors $\left\{\mathbb{E}_{1}, \mathbb{E}_{2}, \mathbb{E}_{3}+\mathbb{E}_{4}\right\}$ together define a two-dimensional subspace.

\subsection{Tetragonal System}

Nine base tensors are required: $\left\{\mathbb{E}_{1}, \mathbb{E}_{2}, \mathbb{E}_{3}, \mathbb{E}_{4}, \mathbb{F}_{1}, \mathbb{F}_{2}, \mathbb{F}_{3}, \mathbb{F}_{4}, \mathbb{G}\right\}$, where the new tensors are a symmetric pair $\mathbb{F}_{1}, \mathbb{F}_{2}$, and a pair that are mutual transposes, $\mathbb{F}_{3}, \mathbb{F}_{4}$,

$$
\mathbb{F}_{1}=\mathbf{W} \otimes \mathbf{W}, \quad \mathbb{F}_{2}=\mathbf{Z} \otimes \mathbf{Z}, \quad \mathbb{F}_{3}=\mathbf{W} \otimes \mathbf{Z}, \quad \mathbb{F}_{4}=\mathbf{Z} \otimes \mathbf{W} .
$$

In comparison with the transversely isotropic system, $\mathbb{F}$ is replaced by the symmetric pair of tensors, i.e., $\mathbb{F}=\mathbb{F}_{1}+\mathbb{F}_{2}$, and the decomposition, of the identity is

$$
\mathbb{I}=\mathbb{E}_{1}+\mathbb{E}_{2}+\mathbb{F}_{1}+\mathbb{F}_{2}+\mathbb{G} \text {. }
$$


The multiplication table of the nine elementary tensors is

\begin{tabular}{l|ccccccccc} 
& $\mathbb{E}_{1}$ & $\mathbb{E}_{2}$ & $\mathbb{E}_{3}$ & $\mathbb{E}_{4}$ & $\mathbb{F}_{1}$ & $\mathbb{F}_{2}$ & $\mathbb{F}_{3}$ & $\mathbb{F}_{4}$ & $\mathbb{G}$ \\
\hline $\mathbb{E}_{1}$ & $\mathbb{E}_{1}$ & $\mathbb{O}$ & $\mathbb{E}_{3}$ & $\mathbb{O}$ & $\mathbb{O}$ & $\mathbb{O}$ & $\mathbb{O}$ & $\mathbb{O}$ & $\mathbb{O}$ \\
$\mathbb{E}_{2}$ & $\mathbb{O}$ & $\mathbb{E}_{2}$ & $\mathbb{O}$ & $\mathbb{E}_{4}$ & $\mathbb{O}$ & $\mathbb{O}$ & $\mathbb{O}$ & $\mathbb{O}$ & $\mathbb{O}$ \\
$\mathbb{E}_{3}$ & $\mathbb{O}$ & $\mathbb{E}_{3}$ & $\mathbb{O}$ & $\mathbb{E}_{1}$ & $\mathbb{O}$ & $\mathbb{O}$ & $\mathbb{O}$ & $\mathbb{O}$ & $\mathbb{O}$ \\
$\mathbb{E}_{4}$ & $\mathbb{E}_{4}$ & $\mathbb{O}$ & $\mathbb{E}_{2}$ & $\mathbb{O}$ & $\mathbb{O}$ & $\mathbb{O}$ & $\mathbb{O}$ & $\mathbb{O}$ & $\mathbb{O}$ \\
$\mathbb{F}_{1}$ & $\mathbb{O}$ & $\mathbb{O}$ & $\mathbb{O}$ & $\mathbb{O}$ & $\mathbb{F}_{1}$ & $\mathbb{O}$ & $\mathbb{F}_{3}$ & $\mathbb{O}$ & $\mathbb{O}$ \\
$\mathbb{F}_{2}$ & $\mathbb{O}$ & $\mathbb{O}$ & $\mathbb{O}$ & $\mathbb{O}$ & $\mathbb{O}$ & $\mathbb{F}_{2}$ & $\mathbb{O}$ & $\mathbb{F}_{4}$ & $\mathbb{O}$ \\
$\mathbb{F}_{3}$ & $\mathbb{O}$ & $\mathbb{O}$ & $\mathbb{O}$ & $\mathbb{O}$ & $\mathbb{O}$ & $\mathbb{F}_{3}$ & $\mathbb{O}$ & $\mathbb{F}_{1}$ & $\mathbb{0}$ \\
$\mathbb{F}_{4}$ & $\mathbb{O}$ & $\mathbb{O}$ & $\mathbb{O}$ & $\mathbb{O}$ & $\mathbb{F}_{4}$ & $\mathbb{O}$ & $\mathbb{F}_{2}$ & $\mathbb{O}$ & $\mathbb{O}$ \\
$\mathbb{G}$ & $\mathbb{O}$ & $\mathbb{O}$ & $\mathbb{O}$ & $\mathbb{O}$ & $\mathbb{O}$ & $\mathbb{O}$ & $\mathbb{O}$ & $\mathbb{O}$ & $\mathbb{G}$
\end{tabular}

Under multiplication the $\mathbb{E}_{i}$ 's and $\mathbb{F}_{i}$ 's decouple from one another and from $\mathbb{G}$. Furthermore, the algebra of the $\mathbb{F}_{i}$ 's is similar to that of the $\mathbb{E}_{i}$ 's. The Euclidean lengths of the new tensors are $\left\|\mathbb{F}_{1}\right\|=\left\|\mathbb{F}_{2}\right\|=1,\left\|\mathbb{F}_{3}+\mathbb{F}_{4}\right\|=\sqrt{2}$.

A general symmetric fourth-order tensor of tetragonal symmetry is given by the six-parameter combination

$$
\mathbb{A}=a \mathbb{E}_{1}+b \mathbb{E}_{2}+c\left(\mathbb{E}_{3}+\mathbb{E}_{4}\right)+p \mathbb{F}_{1}+q \mathbb{F}_{2}+r\left(\mathbb{F}_{3}+\mathbb{F}_{4}\right)+g \mathbb{G} .
$$

Tetragonal symmetry is often represented by six rather than seven independent parameters. Fedorov [4] pointed out that transformation by rotation about the $\mathbf{c}$ axis by angle $\theta$ yields zero for the transformed coefficient $r$ if (see Eq. (9.7) in [4])

$$
\tan 4 \theta=r /(q-p) .
$$

The transformation (38) depends on knowledge of the coefficients. However, it is assumed here that the only properties of the tetragonal symmetry available a priori are the orthogonal crystal axes $\mathbf{a}, \mathbf{b}$ and $\mathbf{c}$. The reduction from seven to six parameters can be achieved after the effective tetragonal material is found, but the axes of the final six-parameter material depend upon the initial moduli. Hence, it is important to retain the seven-parameter representation (37).

\subsection{Trigonal System}

Trigonal symmetry is characterized by three planes of reflection symmetry with normals coplanar and at $120^{\circ}$ to one another. This symmetry class is also known as hexagonal [19] but we refer to it as trigonal since hexagonal symmetry is often used synonymously with transverse isotropy. It is advantageous to choose non-orthogonal basis vectors $\mathbf{a}^{\prime}, \mathbf{b}^{\prime}, \mathbf{c}$ with $\mathbf{a}^{\prime}$ and $\mathbf{b}^{\prime}$ normals to two of the planes and $\mathbf{c}$ perpendicular to them (the pair $\mathbf{a}^{\prime}$ and $\mathbf{b}^{\prime}$ may also be represented in terms of the orthonormal vectors via, for instance, $\left.\mathbf{a}^{\prime}=\mathbf{a}, \mathbf{b}^{\prime}=-\frac{1}{2} \mathbf{a}+\frac{\sqrt{3}}{2} \mathbf{b}\right)$. In order to describe symmetric fourth-order tensors of trigonal symmetry, following [19] we introduce the secondorder tensors

$$
\begin{aligned}
\mathbf{S} & =\sqrt{\frac{2}{3}}\left(\mathbf{a}^{\prime} \otimes \mathbf{b}^{\prime}+\mathbf{b}^{\prime} \otimes \mathbf{a}^{\prime}+\mathbf{a}^{\prime} \otimes \mathbf{a}^{\prime}\right), & \mathbf{T} & =\sqrt{\frac{2}{3}}\left(\mathbf{a}^{\prime} \otimes \mathbf{b}^{\prime}+\mathbf{b}^{\prime} \otimes \mathbf{a}^{\prime}+\mathbf{b}^{\prime} \otimes \mathbf{b}^{\prime}\right), \\
\mathbf{U}^{\prime} & =\frac{1}{\sqrt{2}}\left(\mathbf{a}^{\prime} \otimes \mathbf{c}+\mathbf{c} \otimes \mathbf{a}^{\prime}\right), & \mathbf{V}^{\prime} & =-\frac{1}{\sqrt{2}}\left(\mathbf{b}^{\prime} \otimes \mathbf{c}+\mathbf{c} \otimes \mathbf{b}^{\prime}\right),
\end{aligned}
$$


the two symmetric fourth-order tensors

$$
\begin{aligned}
& \mathbb{R}_{1}=\frac{4}{3}\left(\mathbf{S} \otimes \mathbf{S}+\mathbf{T} \otimes \mathbf{T}-\frac{1}{2} \mathbf{S} \otimes \mathbf{T}-\frac{1}{2} \mathbf{T} \otimes \mathbf{S}\right), \\
& \mathbb{R}_{2}=\frac{4}{3}\left(\mathbf{U}^{\prime} \otimes \mathbf{U}^{\prime}+\mathbf{V}^{\prime} \otimes \mathbf{V}^{\prime}-\frac{1}{2} \mathbf{U}^{\prime} \otimes \mathbf{V}^{\prime}-\frac{1}{2} \mathbf{V}^{\prime} \otimes \mathbf{U}^{\prime}\right),
\end{aligned}
$$

and two pairs of mutually transpose tensors:

$$
\begin{aligned}
& \mathbb{R}_{3}=\frac{4}{3}\left(\mathbf{S} \otimes \mathbf{U}^{\prime}+\mathbf{T} \otimes \mathbf{V}^{\prime}-\frac{1}{2} \mathbf{T} \otimes \mathbf{U}^{\prime}-\frac{1}{2} \mathbf{S} \otimes \mathbf{V}^{\prime}\right), \\
& \mathbb{R}_{4}=\frac{4}{3}\left(\mathbf{U}^{\prime} \otimes \mathbf{S}+\mathbf{V}^{\prime} \otimes \mathbf{T}-\frac{1}{2} \mathbf{U}^{\prime} \otimes \mathbf{T}-\frac{1}{2} \mathbf{V}^{\prime} \otimes \mathbf{S}\right),
\end{aligned}
$$

and

$$
\mathbb{R}_{5}=\frac{2}{\sqrt{3}}\left(\mathbf{S} \otimes \mathbf{V}^{\prime}-\mathbf{T} \otimes \mathbf{U}^{\prime}\right), \quad \mathbb{R}_{6}=\frac{2}{\sqrt{3}}\left(\mathbf{V}^{\prime} \otimes \mathbf{S}-\mathbf{U}^{\prime} \otimes \mathbf{T}\right) .
$$

To make the $\mathbb{R}_{i}$ 's closed under multiplication, we need to further introduce two skew-symmetric tensors

$$
\mathbb{R}_{7}=\frac{2}{\sqrt{3}}(\mathbf{T} \otimes \mathbf{S}-\mathbf{S} \otimes \mathbf{T}), \quad \mathbb{R}_{8}=\frac{2}{\sqrt{3}}\left(\mathbf{U}^{\prime} \otimes \mathbf{V}^{\prime}-\mathbf{V}^{\prime} \otimes \mathbf{U}^{\prime}\right) .
$$

Thus, $\mathbb{E}_{i} \mathbb{R}_{j}=\mathbb{R}_{j} \mathbb{E}_{i}=\mathbb{O}$ for $i=1,2, \ldots, 4$ and $j=1,2, \ldots, 8$, and the multiplication table is ${ }^{2}$

\begin{tabular}{l|cccccccc} 
& $\mathbb{R}_{1}$ & $\mathbb{R}_{2}$ & $\mathbb{R}_{3}$ & $\mathbb{R}_{4}$ & $\mathbb{R}_{5}$ & $\mathbb{R}_{6}$ & $\mathbb{R}_{7}$ & $\mathbb{R}_{8}$ \\
\hline $\mathbb{R}_{1}$ & $\mathbb{R}_{1}$ & $\mathbb{O}$ & $\mathbb{R}_{3}$ & $\mathbb{O}$ & $\mathbb{R}_{5}$ & $\mathbb{O}$ & $\mathbb{R}_{7}$ & $\mathbb{O}$ \\
$\mathbb{R}_{2}$ & $\mathbb{O}$ & $\mathbb{R}_{2}$ & $\mathbb{O}$ & $\mathbb{R}_{4}$ & $\mathbb{O}$ & $\mathbb{R}_{6}$ & $\mathbb{O}$ & $\mathbb{R}_{8}$ \\
$\mathbb{R}_{3}$ & $\mathbb{O}$ & $\mathbb{R}_{3}$ & $\mathbb{O}$ & $\mathbb{R}_{1}$ & $\mathbb{O}$ & $\mathbb{R}_{7}$ & $\mathbb{O}$ & $\mathbb{R}_{5}$ \\
$\mathbb{R}_{4}$ & $\mathbb{R}_{4}$ & $\mathbb{O}$ & $\mathbb{R}_{2}$ & $\mathbb{O}$ & $\mathbb{R}_{8}$ & $\mathbb{O}$ & $\mathbb{R}_{6}$ & $\mathbb{O}$ \\
$\mathbb{R}_{5}$ & $\mathbb{O}$ & $\mathbb{R}_{5}$ & $\mathbb{O}$ & $-\mathbb{R}_{7}$ & $\mathbb{O}$ & $\mathbb{R}_{1}$ & $\mathbb{O}$ & $-\mathbb{R}_{3}$ \\
$\mathbb{R}_{6}$ & $\mathbb{R}_{6}$ & $\mathbb{O}$ & $-\mathbb{R}_{8}$ & $\mathbb{O}$ & $\mathbb{R}_{2}$ & $\mathbb{O}$ & $-\mathbb{R}_{4}$ & $\mathbb{O}$ \\
$\mathbb{R}_{7}$ & $\mathbb{R}_{7}$ & $\mathbb{O}$ & $-\mathbb{R}_{5}$ & $\mathbb{O}$ & $\mathbb{R}_{3}$ & $\mathbb{O}$ & $-\mathbb{R}_{1}$ & $\mathbb{O}$ \\
$\mathbb{R}_{8}$ & $\mathbb{O}$ & $\mathbb{R}_{8}$ & $\mathbb{O}$ & $-\mathbb{R}_{6}$ & $\mathbb{O}$ & $\mathbb{R}_{4}$ & $\mathbb{O}$ & $-\mathbb{R}_{2}$
\end{tabular}

The algebra of the $\mathbb{R}_{i}$ 's is equivalent to that of $2 \times 2$ complex matrices [19]. We note that $\mathbb{R}_{1}=\mathbb{F}$ and $\mathbb{R}_{2}=\mathbb{G}$, and hence the decomposition for the identity tensor is

$$
\mathbb{I}=\mathbb{E}_{1}+\mathbb{E}_{2}+\mathbb{R}_{1}+\mathbb{R}_{2}
$$

The Euclidean lengths of the new tensors are $\left\|\mathbb{R}_{1}\right\|=\left\|\mathbb{R}_{2}\right\|=\sqrt{2},\left\|\mathbb{R}_{3}+\mathbb{R}_{4}\right\|=$ $\left\|\mathbb{R}_{5}+\mathbb{R}_{6}\right\|=2$.

A general symmetric fourth-order tensor of trigonal symmetry is given by the linear combination

$$
\mathbb{A}=a \mathbb{E}_{1}+b \mathbb{E}_{2}+c\left(\mathbb{E}_{3}+\mathbb{E}_{4}\right)+p \mathbb{R}_{1}+q \mathbb{R}_{2}+r\left(\mathbb{R}_{3}+\mathbb{R}_{4}\right)+s\left(\mathbb{R}_{5}+\mathbb{R}_{6}\right) .
$$

2 The multiplication table in [19] contains some typographical errors; specifically the elements $(3,8)$, $(4,5),(5,8),(6,3),(8,4)$ and $(8,6)$ in the original need to be multiplied by -1 . 
4.7 Rhombic, Monoclinic and Triclinic Systems

The algebra for orthorhombic (equivalently orthotropic) symmetry is the same as that of $3 \times 3$ real matrices plus three real numbers. For monoclinic symmetry it is the same as the algebra of pairs of $4 \times 4$ and $2 \times 2$ real matrices. The lowest symmetry, triclinic or no symmetry, has algebra the same as that of $6 \times 6$ real matrices. These low symmetries are probably better defined in terms of their group properties, which also serves as efficient means for Euclidean projection, as described in Section 5.

\subsection{Six-dimensional Representation}

An equivalent six-dimensional matrix format is described for the various elementary tensors. For each symmetry class the six-dimensional tensors possess the same algebraic properties as the fourth-order tensors, with the same multiplication tables and Euclidean lengths.

The six-dimensional vectors $\{\hat{\mathbf{a}}, \hat{\mathbf{b}}, \hat{\mathbf{c}}\}$ represent the second-order tensors $\{\mathbf{a} \otimes \mathbf{a}, \mathbf{b} \otimes \mathbf{b}, \mathbf{c} \otimes \mathbf{c}\}$ that correspond to the axes $\{\mathbf{a}, \mathbf{b}, \mathbf{c}\}$, where according to Eq. (5),

$$
\mathbf{a}=a_{1} \mathbf{e}_{1}+a_{2} \mathbf{e}_{2}+a_{3} \mathbf{e}_{3} \Rightarrow \hat{\mathbf{a}}=\left(a_{1}^{2}, a_{2}^{2}, a_{3}^{2}, \sqrt{2} a_{2} a_{3}, \sqrt{2} a_{3} a_{1}, \sqrt{2} a_{1} a_{2}\right)^{t},
$$

The associated $6 \times 6$ base matrices are given next.

\subsubsection{Isotropic System}

The six-dimensional analogs of $\mathbb{I}, \mathbb{J}$ and $\mathbb{K}$ are $\widehat{\mathbf{I}}$, the $6 \times 6$ identity matrix, and $\widehat{\mathbf{J}}, \widehat{\mathbf{K}}$, where

$$
\widehat{\mathbf{J}}=\frac{1}{3}\left(\hat{\mathbf{e}}_{1}+\hat{\mathbf{e}}_{2}+\hat{\mathbf{e}}_{3}\right)\left(\hat{\mathbf{e}}_{1}+\hat{\mathbf{e}}_{2}+\hat{\mathbf{e}}_{3}\right)^{t}, \quad \widehat{\mathbf{K}}=\widehat{\mathbf{I}}-\widehat{\mathbf{J}}
$$

\subsubsection{Cubic System}

The analogs of $\mathbb{L}$ and $\mathbb{M}$ are

$$
\widehat{\mathbf{L}}=\widehat{\mathbf{I}}-\hat{\mathbf{a}} \hat{\mathbf{a}}^{t}-\hat{\mathbf{b}} \hat{\mathbf{b}}^{t}-\hat{\mathbf{c}} \hat{\mathbf{c}}^{t}, \quad \widehat{\mathbf{M}}=\widehat{\mathbf{K}}-\widehat{\mathbf{L}} .
$$

\subsubsection{Transversely Isotropic System}

Define the six-vectors

$$
\begin{aligned}
& \hat{\mathbf{p}}=\hat{\mathbf{c}}, \quad \hat{\mathbf{q}}=\frac{1}{\sqrt{2}}(\hat{\mathbf{a}}+\hat{\mathbf{b}}), \quad \hat{\mathbf{z}}=\frac{1}{\sqrt{2}}(\hat{\mathbf{a}}-\hat{\mathbf{b}}), \\
& \hat{\mathbf{w}}=\left(\sqrt{2} a_{1} b_{1}, \sqrt{2} a_{2} b_{2}, a \sqrt{2}{ }_{3} b_{3},\left(a_{2} b_{3}+a_{3} b_{2}\right),\left(a_{3} b_{1}+a_{1} b_{3}\right),\left(a_{1} b_{2}+a_{2} b_{1}\right)\right)^{t},
\end{aligned}
$$

then the six-dimensional matrices for the tensors $\mathbb{E}_{1}, \mathbb{E}_{2}, \mathbb{E}_{3}, \mathbb{E}_{4}, \mathbb{F}$ and $\mathbb{G}$ are

$$
\begin{aligned}
\widehat{\mathbf{E}}_{1} & =\hat{\mathbf{p}} \hat{\mathbf{p}}^{t}, \quad \widehat{\mathbf{E}}_{2}=\hat{\mathbf{q}} \hat{\mathbf{q}}^{t}, \quad \widehat{\mathbf{E}}_{3}=\hat{\mathbf{p}} \hat{\mathbf{q}}^{t}, \quad \widehat{\mathbf{E}}_{4}=\hat{\mathbf{q}} \hat{\mathbf{p}}^{t}, \\
\widehat{\mathbf{F}} & =\hat{\mathbf{w}} \hat{\mathbf{w}}^{t}+\hat{\mathbf{z}} \hat{\mathbf{z}}^{t}, \quad \widehat{\mathbf{G}}=\widehat{\mathbf{L}}-\hat{\mathbf{w}} \hat{\mathbf{w}}^{t} .
\end{aligned}
$$




\subsubsection{Tetragonal System}

The six-dimensional representation is as for TI, with the addition

$$
\widehat{\mathbf{F}}_{1}=\hat{\mathbf{w}} \hat{\mathbf{w}}^{t}, \quad \widehat{\mathbf{F}}_{2}=\hat{\mathbf{z}} \hat{\mathbf{z}}^{t} \quad \widehat{\mathbf{F}}_{3}=\hat{\mathbf{w}} \hat{\mathbf{z}}^{t}, \quad \widehat{\mathbf{F}}_{4}=\hat{\mathbf{z}} \hat{\mathbf{w}}^{t} .
$$

The trigonal system can be treated in the same manner using equations similar to Eq. (46) for $\hat{\mathbf{a}}$ and Eq. (49) for $\hat{\mathbf{w}}$, but the details are omitted for brevity.

\section{Euclidean Projection}

Euclidean projection is an essential ingredient for determining the closest tensors in both the Euclidean and the log-Euclidean metrics. It is defined in abstract terms in the next subsection, with the remainder of the section devoted to explicit procedures for the projection.

\subsection{Definition of the Projection}

We wish to find the tensor $\mathbb{C}_{\text {sym }}$ of a specific symmetry class which minimizes the Euclidean distance $\left\|\mathbb{C}-\mathbb{C}_{\text {sym }}\right\|$ of an elasticity tensor of arbitrary symmetry, $\mathbb{C}$, from the particular symmetry. The solution is a Euclidean decomposition

$$
\mathbb{C}=\mathbb{C}_{\text {sym }}+\mathbb{C}_{\perp \text { sym }},
$$

where $\mathbb{C}_{\text {sym }}$ possesses the symmetries appropriate to the symmetry class considered. The complement, or residue [3], is orthogonal to $\mathbb{C}_{\text {sym }}$,

$$
\left\langle\mathbb{C}_{\text {sym }}, \mathbb{C}_{\perp \text { sym }}\right\rangle=0,
$$

and hence

$$
\begin{aligned}
\|\mathbb{C}\|^{2} & =\left\|\mathbb{C}_{\text {sym }}\right\|^{2}+\left\|\mathbb{C}_{\perp \text { sym }}\right\|^{2}, \\
\left\|\mathbb{C}-\mathbb{C}_{\text {sym }}\right\|^{2} & =\left\|\mathbb{C}_{\perp \text { sym }}\right\|^{2} .
\end{aligned}
$$

We illustrate the recursive nature of the Euclidean projection for the special case of isotropy. Any elasticity may be partitioned into orthogonal components as

$$
\begin{aligned}
\mathbb{C} & =\mathbb{C}_{\text {iso }}+\mathbb{C}_{\perp \text { iso }} \\
& =\mathbb{C}_{\text {iso }}+\mathbb{C}_{\text {cub } / \text { iso }}+\mathbb{C}_{\text {tet } / \text { cub }}+\mathbb{C}_{\text {ort } / \text { tet }}+\mathbb{C}_{\text {mon/ort }}+\mathbb{C}_{\perp \text { mon }},
\end{aligned}
$$

where

$$
\mathbb{C}_{\text {sym B/A }} \equiv \mathbb{C}_{\text {sym B }}-\mathbb{C}_{\text {sym A }},
$$

with sym A of higher symmetry than sym B. Eq. (55) partitions $\mathbb{C}$ using the path from no symmetry to isotropy via cubic symmetry, see Figure 1 . An alternative decomposition via hexagonal, gives

$$
\mathbb{C}=\mathbb{C}_{\text {iso }}+\mathbb{C}_{\text {hex } / \text { iso }}+\mathbb{C}_{\text {tet } / \text { hex }}+\mathbb{C}_{\text {ort/tet }}+\mathbb{C}_{\text {mon/ort }}+\mathbb{C}_{\perp \text { mon }} .
$$

The different paths in Figure 1 were identified by Gazis et al. [3] and discussed more recently in greater detail by Chadwick et al. [18]. The idea of sequential 
Figure 1 The sequence of increasing elastic symmetries, from triclinic to isotropic. The number of independent elastic constants are listed. The dashed boxes for trigonal 6 and tetragonal 6 indicate that these are obtained from the lower symmetries by rotation, and do not represent new elastic symmetries. The true symmetries are the eight in solid boxes [18].

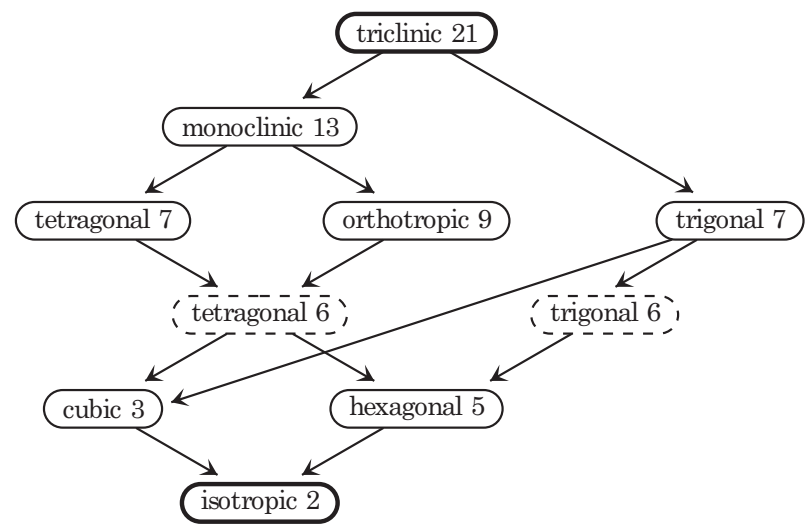

decomposition in the Euclidean norm is not new, e.g., [14, 42], but the explicit projection and complement have not been represented previously.

Details of the recursive Euclidean partition of $\mathbb{C}$ are given in the Appendix. For instance, Eq. (A.30) represents $\|\mathbb{C}\|^{2}$ using three different routes from triclinic to isotropic. In particular, the length of the isotropic projection is given by

$$
\left\|\mathbb{C}_{\text {iso }}\right\|^{2}=9 \kappa^{2}+20 \mu^{2},
$$

where $\kappa$ and $\mu$ are defined in Eq. (23), and the distance of $\mathbb{C}$ from isotropy is given by

$$
\left\|\mathbb{C}_{\perp \text { iso }}\right\|^{2}=\|\mathbb{C}\|^{2}-9 \kappa^{2}-20 \mu^{2} .
$$

It appears that Fedorov [4] was the first to show that the distance from isotropy is minimized if the isotropic tensor is as given in Eq. (22).

The remainder of this section details three alternative projection methods using basis tensors, using symmetry planes or groups, and using 21-dimensional vectors. The projection operators are valid for all symmetries, but the method based on symmetry planes becomes more complicated for the higher symmetries. Conversely, the projection operator using basis tensors becomes simpler at the higher symmetries. The 21-dimensional vector procedure is straightforward but is coordinate dependent.

\subsection{Projection Using a Tensor Basis}

We assume that the set of tensors $\left\{\mathbb{V}_{i} \in \mathbb{E}\right.$ la $\}$ form a linearly independent basis for the symmetry sym in the sense that any elasticity tensor of that symmetry may be expressed uniquely in terms of $N$ linearly independent tensors $\mathbb{V}_{1}, \mathbb{V}_{2}, \ldots \mathbb{V}_{N}$, where $2 \leq N \leq 13$ is the dimension of the space for the material symmetry. We have seen the explicit form of several sets of basis tensors in Section 4, e.g., $N=2$ for isotropic elasticity. $N=13$ corresponds to monoclinic, which is the lowest symmetry apart from triclinic (technically $N=21$ ) which is no symmetry. The $N$ elements of the orthogonal basis are assumed to be independent of the elasticity itself, i.e., they do not require "elasticity distributors", which are necessary for the spectral decomposition of $\mathbb{E}$ la [13]. They depend only on the choice of the planes and/or axes which define the group $G$ of symmetry preserving transformations (see below). The precise form of the basis tensors is irrelevant, all that is required is that they be 
linearly independent in the sense of elements of a vector space, and consequently any tensor with the desired symmetry can be expressed

$$
\mathbb{C}_{\text {sym }}=\sum_{i=1}^{N} a_{i} \mathbb{V}_{i}
$$

Minimizing $\mathrm{d}_{F}^{2}\left(\mathbb{C}, \mathbb{C}_{\text {sym }}\right)$ with respect to the coefficients $a_{i}$ implies

$$
\frac{\partial}{\partial a_{i}}\left\|\mathbb{C}-\mathbb{C}_{\text {sym }}\right\|^{2}=2\left\langle\mathbb{C}, \mathbb{V}_{i}\right\rangle-2\left\langle\mathbb{C}_{\text {sym }}, \mathbb{V}_{i}\right\rangle=0
$$

Hence,

$$
\sum_{j=1}^{N} a_{j}\left\langle\mathbb{V}_{j}, \mathbb{V}_{i}\right\rangle=\left\langle\mathbb{C}, \mathbb{V}_{i}\right\rangle
$$

Let $\mathbf{D}$ be the $N \times N$ symmetric matrix with elements

$$
D_{i j} \equiv\left\langle\mathbb{V}_{i}, \mathbb{V}_{j}\right\rangle
$$

This is invertible by virtue of the linear independence of the basis tensors, and so

$$
a_{i}=\sum_{j=1}^{N} D_{i j}^{-1}\left\langle\mathbb{V}_{j}, \mathbb{C}\right\rangle .
$$

Noting that $\mathbb{C}_{\perp \text { sym }}=\mathbb{C}-\mathbb{C}_{\text {sym }}$, we have

$$
\begin{aligned}
\left\langle\mathbb{C}_{\perp \text { sym }}, \mathbb{C}_{\text {sym }}\right\rangle & =\left\langle\mathbb{C}-\sum_{i=1}^{N} a_{i} \mathbb{V}_{i}, \sum_{j=1}^{N} a_{j} \mathbb{V}_{j}\right\rangle \\
& =\sum_{j=1}^{N} a_{j}\left\langle\mathbb{C}, \mathbb{V}_{j}\right\rangle-\sum_{i=1}^{N} \sum_{j=1}^{N} a_{i} a_{j}\left\langle\mathbb{V}_{i}, \mathbb{V}_{j}\right\rangle=0 .
\end{aligned}
$$

Hence, the partition (60) satisfies the fundamental projection property (52). It is a simple exercise to show that $\mathbb{C}_{\text {sym }}$ of Eq. (60) minimizes the Euclidean distance $\left\|\mathbb{C}-\mathbb{C}_{\text {sym }}\right\|$ iff the coefficients are given by Eq. (64).

The Euclidean projection can also be expressed in terms of tensors of order eight, $P_{\text {sym }}$, such that

$$
\mathbb{C}_{\text {sym }}=P_{\text {sym }} \mathbb{C}, \quad \Leftrightarrow \quad \mathbb{C}_{i j k l}^{\mathrm{sym}}=P_{i j k l m n r s}^{\mathrm{sym}} C_{\text {mnrs }} .
$$

The projector can be expressed in terms of dyadics of basis tensors,

$$
P_{\text {sym }}=\sum_{i, j=1}^{N} D_{i j}^{-1} \mathbb{V}_{i} \otimes \mathbb{V}_{j} .
$$

We now apply these ideas to particular symmetries, focusing only on the higher symmetries since the lower ones (monoclinic, orthorhombic) are relatively trivial and can be handled using the projector of Eq. (71). 
5.3 Projection Operators for Particular Symmetries

The projector for the symmetries described in Section 4 follow naturally from the vectorial formulation. In particular, we note that the $\mathbf{D}$ is diagonal in each case, and

$$
\begin{aligned}
P_{\text {iso }}= & \mathbb{J} \otimes \mathbb{J}+\frac{1}{5} \mathbb{K} \otimes \mathbb{K} \\
P_{\text {cub }}= & \mathbb{J} \otimes \mathbb{J}+\frac{1}{3} \mathbb{L} \otimes \mathbb{L}+\frac{1}{2} \mathbb{M} \otimes \mathbb{M} \\
P_{\text {hex }}= & \mathbb{E}_{1} \otimes \mathbb{E}_{1}+\mathbb{E}_{2} \otimes \mathbb{E}_{2}+\frac{1}{2}\left(\mathbb{E}_{3}+\mathbb{E}_{4}\right) \otimes\left(\mathbb{E}_{3}+\mathbb{E}_{4}\right) \\
& +\frac{1}{2} \mathbb{F} \otimes \mathbb{F}+\frac{1}{2} \mathbb{G} \otimes \mathbb{G}, \\
P_{\text {tet }}= & \mathbb{E}_{1} \otimes \mathbb{E}_{1}+\mathbb{E}_{2} \otimes \mathbb{E}_{2}+\frac{1}{2}\left(\mathbb{E}_{3}+\mathbb{E}_{4}\right) \otimes\left(\mathbb{E}_{3}+\mathbb{E}_{4}\right) \\
& +\mathbb{F}_{1} \otimes \mathbb{F}_{1}+\mathbb{F}_{2} \otimes \mathbb{F}_{2}+\frac{1}{2}\left(\mathbb{F}_{3}+\mathbb{F}_{4}\right) \otimes\left(\mathbb{F}_{3}+\mathbb{F}_{4}\right)+\frac{1}{2} \mathbb{G}_{0} \otimes \mathbb{G} \\
P_{\text {trig }}= & \mathbb{E}_{1} \otimes \mathbb{E}_{1}+\mathbb{E}_{2} \otimes \mathbb{E}_{2}+\frac{1}{2}\left(\mathbb{E}_{3}+\mathbb{E}_{4}\right) \otimes\left(\mathbb{E}_{3}+\mathbb{E}_{4}\right)+\frac{1}{2} \mathbb{R}_{1} \otimes \mathbb{R}_{1} \\
& +\frac{1}{2} \mathbb{R}_{2} \otimes \mathbb{R}_{2}+\frac{1}{4}\left(\mathbb{R}_{3}+\mathbb{R}_{4}\right) \otimes\left(\mathbb{R}_{3}+\mathbb{R}_{4}\right)+\frac{1}{4}\left(\mathbb{R}_{5}+\mathbb{R}_{6}\right) \otimes\left(\mathbb{R}_{5}+\mathbb{R}_{6}\right)
\end{aligned}
$$

Expressions can be given for the lower symmetries, but as discussed, the number of elementary tensors involved is much larger. The form of the projectors in Eq. (68) are independent of the crystal axes, and provide a coordinate-free scheme for Euclidean projection. They can be easily programmed using the six-dimensional matrix representation.

If sym $\mathrm{A}>$ sym $\mathrm{B}$, we may convert $P_{\text {symB }}$ into a form that yields $\mathbb{C}_{\text {sym B }}$ and the complement $\mathbb{C}_{\text {sym B/A }}$ of Eq. (56). A general procedure for achieving this uses the fact that basis tensors for a given symmetry are not unique, and accordingly, alternate forms of $P_{\text {sym }}$ may be found. The decomposition

$$
P_{\text {sym B }}=P_{\text {sym A }}+P_{\text {sym B } / \mathrm{A}},
$$

can be found by a Gram-Schmid type of process. As an example, let sym $\mathrm{A}=$ iso and sym $B=$ cub, and consider the tensor basis for cubic symmetry $\left\{\mathbb{V}_{1}, \mathbb{V}_{2}, \mathbb{V}_{3}\right\}=$ $\{\mathbb{J}, \mathbb{K}, \mathbb{L}-a \mathbb{M}\}$, where $a$ is a free parameter. Requiring that $\mathbf{D}$ is diagonal implies $a=3 / 2$, and consequently we obtain

$$
P_{\text {cub }}=\mathbb{J} \otimes \mathbb{J}+\frac{1}{5} \mathbb{K} \otimes \mathbb{K}+\frac{1}{30}(2 \mathbb{L}-3 \mathbb{M}) \otimes(2 \mathbb{L}-3 \mathbb{M})=P_{\text {iso }}+P_{\text {cub } / \text { iso }}
$$

The analogous partition for isotropy and transverse isotropy is described in Appen-

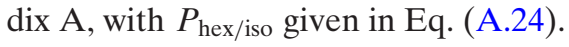


5.4 Projection Using the Transformation Group

Let $G$ be the group of transformations for the material symmetry. $G \subset S O$ (3), the space of three-dimensional special (or proper) orthogonal transformations. Under the change of basis associated with $\mathbf{Q} \in S O(3)$, an elasticity tensor transforms $\mathbb{C} \rightarrow$ $\mathbb{C}^{\prime}$ where $C_{i j k l}^{\prime}=Q_{i p} Q_{j q} Q_{k r} Q_{l s} C_{p q r s}$. The projection is

$$
C_{i j k l}^{\mathrm{sym}}=\frac{1}{n} \sum_{m=1}^{n} Q_{i p}^{(m)} Q_{j q}^{(m)} Q_{k r}^{(m)} Q_{l s}^{(m)} C_{p q r s},
$$

where $n$ is the order of the group $G$, and $\mathbf{Q}^{(m)}$, are elements of the group.

The order of the groups are as follows (see Table 6 of [35]): triclinic 1, monoclinic 2 , trigonal 6 , orthorhombic 4 , tetragonal 8 , transverse isotropy $\infty+2$, and isotropy $\infty^{3}$. The projection defined by Eq. (71) was discussed by, among others, Gazis et al. [3], and has been applied to ultrasonic data by François et al. [47].

The six-dimensional version of the projection (71) is

$$
\widehat{\mathbf{C}}_{\mathrm{sym}}=\frac{1}{n} \sum_{m=1}^{n} \widehat{\mathbf{Q}}^{(m)} \widehat{\mathbf{C}}^{(m)^{t}}
$$

where $\widehat{\mathbf{Q}}^{(m)} \in S O(6)$ is the orthogonal second-order tensor corresponding to $\mathbf{Q}^{(m)}$, satisfying $\widehat{\mathbf{Q}} \widehat{\mathbf{Q}}^{t}=\widehat{\mathbf{Q}}^{t} \widehat{\mathbf{Q}}=\widehat{\mathbf{I}}$. A necessary and sufficient condition that $\widehat{\mathbf{C}}_{\text {sym }}$ has the correct symmetry is that $\widehat{\mathbf{Q}}^{(j)} \widehat{\mathbf{C}}_{\text {sym }} \widehat{\mathbf{Q}}^{(j)^{t}}$, corresponding to the transformation $\mathbf{Q}^{(j)}$, is also a member of the symmetry class. Thus, for $j=1,2, \ldots, n$,

$$
\widehat{\mathbf{Q}}^{(j)} \widehat{\mathbf{C}}_{\mathrm{sym}} \widehat{\mathbf{Q}}^{(j)^{t}}=\frac{1}{n} \sum_{i=1}^{n} \widehat{\mathbf{Q}}^{(j)} \widehat{\mathbf{Q}}^{(i)} \widehat{\mathbf{C}}^{(i)^{t}} \widehat{\mathbf{Q}}^{(j)^{t}}=\frac{1}{n} \sum_{k=1}^{n} \widehat{\mathbf{Q}}^{(k)} \widehat{\mathbf{C}}^{(k)^{t}} .
$$

The latter identity follows from $\widehat{\mathbf{Q}}^{(j)} \widehat{\mathbf{Q}}^{(i)}=\widehat{\mathbf{Q}}^{(k)}$ and the fact that the $\widehat{\mathbf{Q}}^{(i)}$ form a group of order $n$. This proves that $\mathbb{C}_{\text {sym }}$ defined in Eq. (71) is indeed an element of the symmetry class.

The projection formula (71) or (72) may be effected in several ways, e.g., by referring to the extensive lists of the $6 \times 6$ matrices $\widehat{\mathbf{Q}}^{(i)}$ provided by Cowin and Mehrabadi [35]. We note that each $\mathbf{Q}^{(i)}$ can be decomposed into a product of reflection operators [35]. The fundamental operator is $\mathbf{R}(\mathbf{n}) \equiv \mathbf{I}-2 \mathbf{n} \otimes \mathbf{n}$, where the unit vector $\mathbf{n}$ is normal to the plane. Chadwick et al. [18] provide a complete description of these sets and groups. It is also possible to represent the group of transformations in terms of elements each corresponding to a single rotation $[18,35]$. The equivalence between reflection operator and rotations follows from the identity $\mathbf{Q}(\mathbf{e}, \pi)=-\mathbf{R}(\mathbf{e})$, where $\mathbf{Q}(\mathbf{n}, \theta) \in S O(3)$ defines rotation about $\mathbf{n}$ by angle $\theta$.

We illustrate this general procedure with an example.

\subsubsection{Example: Projection onto Monoclinic Symmetry}

There is only one plane of symmetry [18], with normal c, say, and $n=2$ group elements $G=\{\mathbf{I},-\mathbf{R}(\mathbf{c})\}$. Using Eq. (71) we have

$$
C_{i j k l}^{\mathrm{mon}}=\frac{1}{2} C_{i j k l}+\frac{1}{2} R_{i p}(\mathbf{c}) R_{j q}(\mathbf{c}) R_{k r}(\mathbf{c}) R_{l s}(\mathbf{c}) C_{p q r s} .
$$


For instance, let $\mathbf{c}=\mathbf{e}_{3}$, and using the explicit form of $\widehat{\mathbf{R}}\left(\mathbf{e}_{3}\right)$ from Eq. (54) of [35] (where it is called $\widehat{\mathbf{R}}^{(3)}$ ),

$$
\widehat{\mathbf{R}}\left(\mathbf{e}_{3}\right)=\operatorname{diag}(1,1,1,-1,-1,1),
$$

then Eq. (72) gives

$$
\widehat{\mathbf{C}}_{\text {mon }}=\frac{1}{2} \widehat{\mathbf{C}}+\frac{1}{2} \widehat{\mathbf{R}}\left(\mathbf{e}_{3}\right) \widehat{\mathbf{C}} \widehat{\mathbf{R}}\left(\mathbf{e}_{3}\right)=\left(\begin{array}{cccccc}
\hat{c}_{11} & \hat{c}_{12} & \hat{c}_{13} & 0 & 0 & \hat{c}_{16} \\
& \hat{c}_{22} & \hat{c}_{23} & 0 & 0 & \hat{c}_{26} \\
& & \hat{c}_{33} & 0 & 0 & \hat{c}_{36} \\
& & & \hat{c}_{44} & \hat{c}_{45} & 0 \\
\mathrm{~S} & \mathrm{Y} & \mathrm{M} & & \hat{c}_{55} & 0 \\
& & & & & \\
& & & & & \hat{c}_{66}
\end{array}\right) .
$$

The example illustrates that the group projection method is practical if the order $n$ of the group $G$ is not large. This is the case for monoclinic, orthorhombic and perhaps trigonal systems, but even for these symmetries it is simpler to implement the algorithm by machine. It is not practical for isotropy and transverse isotropy, each with an infinity of symmetry planes. Application of Eq. (71) for cubic isotropy is unwieldy although has been performed [47]. The explicit orthogonal basis method of Eqs. (60) and (64) provides a simpler procedure for the higher symmetries.

\subsection{Projections Using 21-dimensional Vectors}

The projection is simplest for this representation of $\mathbb{E}$ la because the elastic moduli are explicitly represented as a vector. The operator is a $21 \times 21$ matrix $P_{\text {sym }}$, and the closest elasticity is

$$
X_{\text {sym }}=P_{\text {sym }} X,
$$

where $X$ is defined in Eq. (9). The various projection matrices can be read off from the formulas of Appendix A. Thus, the projector for cubic symmetry is

$$
p_{\text {cub }}=\left(\begin{array}{ccccccccc}
\frac{1}{3} & \frac{1}{3} & \frac{1}{3} & 0 & 0 & 0 & 0 & 0 & 0 \\
\frac{1}{3} & \frac{1}{3} & \frac{1}{3} & 0 & 0 & 0 & 0 & 0 & 0 \\
\frac{1}{3} & \frac{1}{3} & \frac{1}{3} & 0 & 0 & 0 & 0 & 0 & 0 \\
0 & 0 & 0 & \frac{1}{3} & \frac{1}{3} & \frac{1}{3} & 0 & 0 & 0 \\
0 & 0 & 0 & \frac{1}{3} & \frac{1}{3} & \frac{1}{3} & 0 & 0 & 0 \\
0 & 0 & 0 & \frac{1}{3} & \frac{1}{3} & \frac{1}{3} & 0 & 0 & 0 \\
0 & 0 & 0 & 0 & 0 & 0 & \frac{1}{3} & \frac{1}{3} & \frac{1}{3} \\
0 & 0 & 0 & 0 & 0 & 0 & \frac{1}{3} & \frac{1}{3} & \frac{1}{3} \\
0 & 0 & 0 & 0 & 0 & 0 & \frac{1}{3} & \frac{1}{3} & \frac{1}{3}
\end{array}\right) .
$$

Browaeys and Chevrot [14] derived $P_{\text {sym }}$ for isotropy, transverse isotropy (hexagonal symmetry), tetragonal, orthorhombic and monoclinic symmetry. They found that in all cases except monoclinic that the non-zero part of $P_{\text {sym }}$ reduces to a $9 \times 9$ matrix 
$p_{\text {sym }}$, as in Eq. (78). However, it should be noted that this is not true for the tetragonal projector derived here, see Eq. (A.6), for reasons discussed in Section 4.5. Nor it is true for the trigonal projector, see Eq. (A.10).

\section{Exponential, Logarithm and Square Root of Symmetric Fourth-order Tensors}

Unlike the Euclidean projection for the Frobenius norm, finding minimizers for the logarithmic and Riemannian distance functions involves evaluation of analytic functions with symmetric fourth-order tensor arguments. In this section we derive the necessary expressions with emphasis on exponential, logarithm and square root functions that will be used to obtain the closest elastic tensors in Section 7. For this purpose, let $h$ denote the analytic function given by its power series

$$
h(x)=\sum_{m=0}^{\infty} a_{m} x^{m} .
$$

The higher symmetries are simpler, and are considered first.

\subsection{Isotropic System}

Using Eqs. (20) and (21), for $\mathbb{A}$ defined in Eq. (18) we have

$$
\begin{aligned}
h(\mathbb{A}) & =\sum_{m=0}^{\infty} a_{m}(a \mathbb{J}+b \mathbb{K})^{m}=a_{0} \mathbb{I}+\sum_{m=1}^{\infty} a_{m}\left(a^{m} \mathbb{J}+b^{m} \mathbb{K}\right) \\
& =a_{0} \mathbb{I}+\sum_{m=0}^{\infty} a_{m}\left(a^{m} \mathbb{J}+b^{m} \mathbb{K}\right)-a_{0}(\mathbb{J}+\mathbb{K})=h(a) \mathbb{J}+h(b) \mathbb{K} .
\end{aligned}
$$

It follows that

$$
\exp \mathbb{A}=e^{a} \mathbb{J}+e^{b} \mathbb{K}
$$

When $a \neq 0$ and $b \neq 0$, the symmetric tensor $\mathbb{A}$ is invertible and its inverse is

$$
\mathbb{A}^{-1}=\frac{1}{a} \mathbb{J}+\frac{1}{b} \mathbb{K}
$$

The tensor $\mathbb{A}$ is positive definite if $a>0$ and $b>0$, with square root given by

$$
\mathbb{A}^{1 / 2}=\sqrt{a} \mathbb{J}+\sqrt{b} \mathbb{K}
$$

and logarithm

$$
\log \mathbb{A}=\ln a \mathbb{J}+\ln b \mathbb{K} .
$$


6.2 Cubic System

Using Eqs. (26) 1 and (27), for $\mathbb{A}$ defined in Eq. (28) we have

$$
\begin{aligned}
h(\mathbb{A}) & =\sum_{m=0}^{\infty} a_{0}(a \mathbb{J}+b \mathbb{L}+c \mathbb{M})^{m} \\
& =a_{0} \mathbb{I}+\sum_{m=1}^{\infty} a_{m}\left(a^{m} \mathbb{J}+b^{m} \mathbb{L}+c^{m} \mathbb{M}\right) \\
& =a_{0} \mathbb{I}+\sum_{m=0}^{\infty} a_{m}\left(a^{m} \mathbb{J}+b^{m} \mathbb{L}+c^{m} \mathbb{M}\right)-a_{0}(\mathbb{J}+\mathbb{L}+\mathbb{M}) \\
& =h(a) \mathbb{J}+h(b) \mathbb{L}+h(c) \mathbb{M} .
\end{aligned}
$$

In particular,

$$
\exp (\mathbb{A})=e^{a} \mathbb{J}+e^{b} \mathbb{L}+e^{c} \mathbb{M} .
$$

When $a \neq 0, b \neq 0$ and $c \neq 0$, the symmetric tensor $\mathbb{A}$ is invertible and its inverse is given by

$$
\mathbb{A}^{-1}=\frac{1}{a} \mathbb{J}+\frac{1}{b} \mathbb{L}+\frac{1}{c} \mathbb{M} .
$$

If $a>0, b>0$ and $c>0$ then $\mathbb{A}$ is positive definite. Its square root is

$$
\mathbb{A}^{1 / 2}=\sqrt{a} \mathbb{J}+\sqrt{b} \mathbb{L}+\sqrt{c} \mathbb{M},
$$

and its logarithm is given by

$$
\log \mathbb{A}=\ln a \mathbb{J}+\ln b \mathbb{L}+\ln c \mathbb{M} .
$$

\subsection{Transversely Isotropic System}

Using Eqs. (32) and (31), for $\mathbb{A}$ defined in Eq. (33) we have

$$
\begin{aligned}
h(\mathbb{A}) & =\sum_{m=0}^{\infty} a_{m}\left[a \mathbb{E}_{1}+b \mathbb{E}_{2}+c\left(\mathbb{E}_{3}+\mathbb{E}_{4}\right)+f \mathbb{F}+g \mathbb{G}\right]{ }^{m} \\
& =a_{0} \mathbb{I}+\sum_{m=1}^{\infty} a_{m}\left[\left(a \mathbb{E}_{1}+b \mathbb{E}_{2}+c\left(\mathbb{E}_{3}+\mathbb{E}_{4}\right)\right)^{m}+f^{m} \mathbb{F}+g^{m} \mathbb{G}\right] \\
& =\sum_{m=0}^{\infty} a_{m}\left(a \mathbb{E}_{1}+b \mathbb{E}_{2}+c\left(\mathbb{E}_{3}+\mathbb{E}_{4}\right)\right)^{m}+h(f) \mathbb{F}+h(g) \mathbb{G}-a_{0}(\mathbb{F}+\mathbb{G}) \\
& =h(0)\left(\mathbb{E}_{1}+\mathbb{E}_{2}-\mathbb{I}\right)+h\left(a \mathbb{E}_{1}+b \mathbb{E}_{2}+c\left(\mathbb{E}_{3}+\mathbb{E}_{4}\right)\right)+h(f) \mathbb{F}+h(g) \mathbb{G} .
\end{aligned}
$$

Contrary to the two previous classes of material symmetries, the upper left $4 \times 4$ multiplication table for the elementary tensors of a transversely isotropic medium is not diagonal. This fact makes the algebra of transversely isotropic tensors a bit more 
difficult. Fortunately, Walpole [19] showed that the algebra for $\mathbb{E}_{1}, \mathbb{E}_{2} \mathbb{E}_{3}$ and $\mathbb{E}_{4}$ is equivalent to the algebra of $2 \times 2$ matrices. The details are described in Appendix B.

For the sake of simplicity of notation, we use the symbol a to denote the quadruple $(a, b, c, d)$ and introduce the functions

$$
\begin{aligned}
& \alpha(\mathbf{a})=\frac{1}{2}(a+b), \\
& \beta(\mathbf{a})=\frac{1}{2}(a-b), \\
& \gamma(\mathbf{a})=\frac{1}{2} \sqrt{(a-b)^{2}+4\left(c^{2}+d^{2}\right)}, \\
& \delta(\mathbf{a})=\sqrt{a b-c^{2}-d^{2}} \quad\left(\text { if } a b-c^{2}-d^{2} \geq 0\right) .
\end{aligned}
$$

The variable $d$ is not needed in this subsection, so we take $d=0$. We used it to keep the number of auxiliary functions to a minimum.

The procedure is to diagonalize the compound tensor argument in Eq. (89),

$$
\mathbb{B} \equiv a \mathbb{E}_{1}+b \mathbb{E}_{2}+c\left(\mathbb{E}_{3}+\mathbb{E}_{4}\right)=(\alpha(\mathbf{a})+\gamma(\mathbf{a})) \mathbb{E}_{+}+(\alpha(\mathbf{a})-\gamma(\mathbf{a})) \mathbb{E}_{-},
$$

where

$$
\mathbb{E}_{ \pm}=\frac{1}{2}\left(\mathbb{E}_{1}+\mathbb{E}_{2}\right) \pm \frac{1}{2}\left[\cos \psi(\mathbf{a})\left(\mathbb{E}_{1}-\mathbb{E}_{2}\right)+\sin \psi(\mathbf{a})\left(\mathbb{E}_{3}+\mathbb{E}_{4}\right)\right]
$$

with $\psi(\mathbf{a})$ defined by

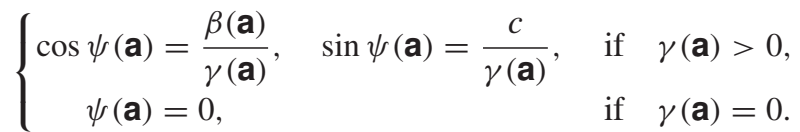

The derivation is apparent from the results below, and explained in detail in Appendix B. The central feature of this decomposition is that $\mathbb{E}_{ \pm}$satisfy $\mathbb{E}_{ \pm} \mathbb{E}_{ \pm}=$ $\mathbb{E}_{ \pm}, \mathbb{E}_{+} \mathbb{E}_{-}=\mathbb{O}, \mathbb{E}_{+}+\mathbb{E}_{-}=\mathbb{E}_{1}+\mathbb{E}_{2}$, and consequently any function, $h$, of the tensor $\mathbb{B}$ may be expressed

$$
h(\mathbb{B})=h(\alpha(\mathbf{a})+\gamma(\mathbf{a})) \mathbb{E}_{+}+h(\alpha(\mathbf{a})-\gamma(\mathbf{a})) \mathbb{E}_{-}+h(0)\left(\mathbb{I}-\mathbb{E}_{1}-\mathbb{E}_{2}\right),
$$

and hence

$$
h(\mathbb{A})=h(\alpha(\mathbf{a})+\gamma(\mathbf{a})) \mathbb{E}_{+}+h(\alpha(\mathbf{a})-\gamma(\mathbf{a})) \mathbb{E}_{-}+h(f) \mathbb{F}+h(g) \mathbb{G} .
$$

Converting back to the $\mathbb{E}_{i}$ 's gives

$$
h(\mathbb{A})=h^{+}(\mathbf{a})\left(\mathbb{E}_{1}+\mathbb{E}_{2}\right)+h^{-}(\mathbf{a})\left[\beta(\mathbf{a})\left(\mathbb{E}_{1}-\mathbb{E}_{2}\right)+c\left(\mathbb{E}_{3}+\mathbb{E}_{4}\right)\right]+h(f) \mathbb{F}+h(g) \mathbb{G},
$$

where

$$
h^{+}(\mathbf{a})=\frac{h(\alpha(\mathbf{a})+\gamma(\mathbf{a}))+h(\alpha(\mathbf{a})-\gamma(\mathbf{a}))}{2},
$$

and

$$
h^{-}(\mathbf{a})= \begin{cases}\frac{h(\alpha(\mathbf{a})+\gamma(\mathbf{a}))-h(\alpha(\mathbf{a})-\gamma(\mathbf{a}))}{2 \gamma(\mathbf{a})}, & \text { if } \gamma(\mathbf{a})>0, \\ h^{\prime}(\alpha(\mathbf{a})), & \text { if } \gamma(\mathbf{a})=0 .\end{cases}
$$


The results of Appendix B and above imply

$$
\exp (\mathbb{A})=\mathfrak{e}_{1}(\mathbf{a}) \mathbb{E}_{1}+\mathfrak{e}_{2}(\mathbf{a}) \mathbb{E}_{2}+\mathfrak{e}_{3}(\mathbf{a})\left(\mathbb{E}_{3}+\mathbb{E}_{4}\right)+e^{f} \mathbb{F}+e^{g} \mathbb{G}
$$

where

$$
\begin{aligned}
& \mathfrak{e}_{1}(\mathbf{a})=\exp (\alpha(\mathbf{a}))[\cosh (\gamma(\mathbf{a}))+\beta(\mathbf{a}) \operatorname{sinhc}(\gamma(\mathbf{a}))], \\
& \mathfrak{e}_{2}(\mathbf{a})=\exp (\alpha(\mathbf{a}))[\cosh (\gamma(\mathbf{a}))-\beta(\mathbf{a}) \operatorname{sinhc}(\gamma(\mathbf{a}))], \\
& \mathfrak{e}_{3}(\mathbf{a})=c \exp (\alpha(\mathbf{a})) \operatorname{sinhc}(\gamma(\mathbf{a})),
\end{aligned}
$$

and $\sinh c(\cdot)$ is the hyperbolic sine cardinal function defined by

$$
\operatorname{sinhc}(x)= \begin{cases}1 & \text { if } x=0, \\ \frac{\sinh (x)}{x} & \text { otherwise. }\end{cases}
$$

Note that $\operatorname{sinhc}(x)$ is continuous at 0 .

When $a b-c^{2} \neq 0, f \neq 0$ and $g \neq 0$, the symmetric tensor $\mathbb{A}$ is invertible and

$$
\mathbb{A}^{-1}=\frac{1}{a b-c^{2}}\left[b \mathbb{E}_{1}+a \mathbb{E}_{2}-c\left(\mathbb{E}_{3}+\mathbb{E}_{4}\right)\right]+\frac{1}{f} \mathbb{F}+\frac{1}{g} \mathbb{G} .
$$

$\mathbb{A}$ is positive definite if $a>0, b>0, a b-c^{2}>0, f>0$ and $g>0$, with square root

$$
\begin{aligned}
\mathbb{A}^{1 / 2}= & \frac{1}{\sqrt{2(\alpha(\mathbf{a})+\delta(\mathbf{a}))}}\left[(a+\delta(\mathbf{a})) \mathbb{E}_{1}+(b+\delta(\mathbf{a})) \mathbb{E}_{2}+c\left(\mathbb{E}_{3}+\mathbb{E}_{4}\right)\right] \\
& +\sqrt{f} \mathbb{F}+\sqrt{g} \mathbb{G},
\end{aligned}
$$

and logarithm

$$
\log \mathbb{A}=\mathfrak{l}_{1}(\mathbf{a}) \mathbb{E}_{1}+\mathfrak{l}_{2}(\mathbf{a}) \mathbb{E}_{2}+\mathfrak{l}_{3}(\mathbf{a})\left(\mathbb{E}_{3}+\mathbb{E}_{4}\right)+\ln f \mathbb{F}+\ln g \mathbb{G},
$$

where

$$
\begin{aligned}
& \mathfrak{l}_{1}(\mathbf{a})=\ln \delta(\mathbf{a})+\beta(\mathbf{a}) \ell(\mathbf{a}), \\
& \mathfrak{l}_{2}(\mathbf{a})=\ln \delta(\mathbf{a})-\beta(\mathbf{a}) \ell(\mathbf{a}), \\
& \mathfrak{l}_{3}(\mathbf{a})=c \ell(\mathbf{a}),
\end{aligned}
$$

with the function $\ell(\cdot)$ defined by

$$
\ell(\mathbf{a})= \begin{cases}\frac{1}{\alpha(\mathbf{a})} & \text { if } \gamma(\mathbf{a})=0, \\ \frac{1}{2 \gamma(\mathbf{a})} \ln \frac{\alpha(\mathbf{a})+\gamma(\mathbf{a})}{\alpha(\mathbf{a})-\gamma(\mathbf{a})} & \text { otherwise. }\end{cases}
$$

Note that for $\gamma(\mathbf{a}) \neq 0$ we have $\frac{1}{2 \gamma(\mathbf{a})} \ln \frac{\alpha(\mathbf{a})+\gamma(\mathbf{a})}{\alpha(\mathbf{a})-\gamma(\mathbf{a})}=\frac{1}{\gamma(\mathbf{a})} \tanh ^{-1} \frac{\gamma(\mathbf{a})}{\alpha(\mathbf{a})}$ and that the limit of this expression is $1 / \alpha(\mathbf{a})$ as $\gamma(\mathbf{a})$ goes to zero.

\subsection{Tetragonal System}

Using the decomposition (35) and the multiplication table (36), an analysis similar to that used for the transversely isotropic system implies that for a tetragonal tensor $\mathbb{A}$ as defined in Eq. (37), 


$$
\begin{aligned}
h(\mathbb{A})= & h^{+}(\mathbf{a})\left(\mathbb{E}_{1}+\mathbb{E}_{2}\right)+h^{-}(\mathbf{a})\left[\beta(\mathbf{a})\left(\mathbb{E}_{1}-\mathbb{E}_{2}\right)+c\left(\mathbb{E}_{3}+\mathbb{E}_{4}\right)\right] \\
& +h^{+}(\mathbf{p})\left(\mathbb{F}_{1}+\mathbb{F}_{2}\right)+h^{-}(\mathbf{p})\left[\beta(\mathbf{p})\left(\mathbb{F}_{1}-\mathbb{F}_{2}\right)+r\left(\mathbb{F}_{3}+\mathbb{F}_{4}\right)\right]+h(g) \mathbb{G}
\end{aligned}
$$

As before, $\mathbf{a}=(a, b, c, d)$ (with $d=0)$ and $\mathbf{p}=(p, q, r, s)($ with $s=0)$.

Thus,

$$
\begin{aligned}
\exp (\mathbb{A})= & \mathfrak{e}_{1}(\mathbf{a}) \mathbb{E}_{1}+\mathfrak{e}_{2}(\mathbf{a}) \mathbb{E}_{2}+\mathfrak{e}_{3}(\mathbf{a})\left(\mathbb{E}_{3}+\mathbb{E}_{4}\right) \\
& +\mathfrak{e}_{1}(\mathbf{p}) \mathbb{F}_{1}+\mathfrak{e}_{2}(\mathbf{p}) \mathbb{F}_{2}+\mathfrak{e}_{3}(\mathbf{p})\left(\mathbb{F}_{3}+\mathbb{F}_{4}\right)+e^{g} \mathbb{G}
\end{aligned}
$$

The tensor $\mathbb{A}$ is invertible if $a b-c^{2} \neq 0, p q-r^{2} \neq 0$ and $g \neq 0$, and

$$
\begin{aligned}
\mathbb{A}^{-1}= & \frac{1}{a b-c^{2}}\left[b \mathbb{E}_{1}+a \mathbb{E}_{2}-c\left(\mathbb{E}_{3}+\mathbb{E}_{4}\right)\right] \\
& +\frac{1}{p q-r^{2}}\left[q \mathbb{F}_{1}+p \mathbb{F}_{2}-r\left(\mathbb{F}_{3}+\mathbb{F}_{4}\right)\right]+\frac{1}{g} \mathbb{G} .
\end{aligned}
$$

$\mathbb{A}$ is positive definite if $a>0, b>0, a b-c^{2}>0, p>0, q>0, p q-r^{2}>0$ and $g>0$, with square root

$$
\begin{aligned}
\mathbb{A}^{1 / 2}= & \frac{1}{\sqrt{2(\alpha(\mathbf{a})+\delta(\mathbf{a}))}}\left[(a+\delta(\mathbf{a})) \mathbb{E}_{1}+(b+\delta(\mathbf{a})) \mathbb{E}_{2}+c\left(\mathbb{E}_{3}+\mathbb{E}_{4}\right)\right] \\
& +\frac{1}{\sqrt{2(\alpha(\mathbf{p})+\delta(\mathbf{p}))}}\left[(p+\delta(\mathbf{p})) \mathbb{F}_{1}+(q+\delta(\mathbf{p})) \mathbb{F}_{2}+r\left(\mathbb{F}_{3}+\mathbb{F}_{4}\right)\right]+\sqrt{g} \mathbb{G}
\end{aligned}
$$

and logarithm

$$
\begin{aligned}
\log \mathbb{A}= & \mathfrak{l}_{1}(\mathbf{a}) \mathbb{E}_{1}+\mathfrak{l}_{2}(\mathbf{a}) \mathbb{E}_{2}+\mathfrak{l}_{3}(\mathbf{a})\left(\mathbb{E}_{3}+\mathbb{E}_{4}\right) \\
& +\mathfrak{l}_{1}(\mathbf{p}) \mathbb{F}_{1}+\mathfrak{l}_{2}(\mathbf{p}) \mathbb{F}_{2}+\mathfrak{l}_{3}(\mathbf{p})\left(\mathbb{F}_{3}+\mathbb{F}_{4}\right)+\ln g \mathbb{G} .
\end{aligned}
$$

\subsection{Trigonal System}

The decomposition (44) and the multiplication table (43) yield for $\mathbb{A}$ defined in Eq. (45)

$$
\begin{aligned}
h(\mathbb{A})= & h^{+}(\mathbf{a})\left(\mathbb{E}_{1}+\mathbb{E}_{2}\right)+h^{-}(\mathbf{a})\left[\beta(\mathbf{a})\left(\mathbb{E}_{1}-\mathbb{E}_{2}\right)+c\left(\mathbb{E}_{3}+\mathbb{E}_{4}\right)\right] \\
& +h^{+}(\mathbf{p})\left(\mathbb{R}_{1}+\mathbb{R}_{2}\right)+h^{-}(\mathbf{p})\left[\beta(\mathbf{p})\left(\mathbb{R}_{1}-\mathbb{R}_{2}\right)+r\left(\mathbb{R}_{3}+\mathbb{R}_{4}\right)+s\left(\mathbb{R}_{5}+\mathbb{R}_{6}\right)\right],
\end{aligned}
$$

where, as before, $\mathbf{a}=(a, b, c, d)$ (with $d=0)$ and $\mathbf{p}=(p, q, r, s)$ (here $s$ need not be zero).

Using these results we have

$$
\begin{aligned}
\exp (\mathbb{A})= & \mathfrak{e}_{1}(\mathbf{a}) \mathbb{E}_{1}+\mathfrak{e}_{2}(\mathbf{a}) \mathbb{E}_{2}+\mathfrak{e}_{3}(\mathbf{a})\left(\mathbb{E}_{3}+\mathbb{E}_{4}\right) \\
& +\mathfrak{e}_{1}(\mathbf{p}) \mathbb{R}_{1}+\mathfrak{e}_{2}(\mathbf{p}) \mathbb{R}_{2}+\mathfrak{e}_{3}(\mathbf{p})\left(\mathbb{R}_{3}+\mathbb{R}_{4}\right)+\mathfrak{e}_{4}(\mathbf{p})\left(\mathbb{R}_{5}+\mathbb{R}_{6}\right)
\end{aligned}
$$


where $\mathfrak{e}_{i}(\cdot), i=1,2,3$ are as defined in Eq. (97) and $\mathfrak{e}_{4}(\mathbf{p})=s \exp (\alpha(\mathbf{p})) \operatorname{sinhc}(\gamma(\mathbf{p}))$. The tensor $\mathbb{A}$ is invertible if $a b-c^{2} \neq 0$ and $p q-r^{2}-s^{2} \neq 0$, with

$$
\begin{aligned}
\mathbb{A}^{-1}= & \frac{1}{a b-c^{2}}\left[b \mathbb{E}_{1}+a \mathbb{E}_{2}-c\left(\mathbb{E}_{3}+\mathbb{E}_{4}\right)\right] \\
& +\frac{1}{p q-r^{2}-s^{2}}\left[q \mathbb{R}_{1}+p \mathbb{R}_{2}-r\left(\mathbb{R}_{3}+\mathbb{R}_{4}\right)-s\left(\mathbb{R}_{5}+\mathbb{R}_{6}\right)\right]
\end{aligned}
$$

$\mathbb{A}$ is positive definite if $a>0, b>0, a b-c^{2}>0, p>0, q>0$ and $p q-r^{2}-s^{2}>0$, with square root

$$
\begin{aligned}
\mathbb{A}^{1 / 2}= & \frac{1}{\sqrt{2(\alpha(\mathbf{a})+\delta(\mathbf{a}))}}\left[(a+\delta(\mathbf{a})) \mathbb{E}_{1}+(b+\delta(\mathbf{a})) \mathbb{E}_{2}+c\left(\mathbb{E}_{3}+\mathbb{E}_{4}\right)\right] \\
& +\frac{1}{\sqrt{2(\alpha(\mathbf{p})+\delta(\mathbf{p}))}}\left[(p+\delta(\mathbf{p})) \mathbb{R}_{1}+(q+\delta(\mathbf{p})) \mathbb{R}_{2}+r\left(\mathbb{R}_{3}+\mathbb{R}_{4}\right)+s\left(\mathbb{R}_{5}+\mathbb{R}_{6}\right)\right]
\end{aligned}
$$

and logarithm

$$
\begin{aligned}
\log \mathbb{A}= & \mathfrak{l}_{1}(\mathbf{a}) \mathbb{E}_{1}+\mathfrak{l}_{2}(\mathbf{a}) \mathbb{E}_{2}+\mathfrak{l}_{3}(\mathbf{a})\left(\mathbb{E}_{3}+\mathbb{E}_{4}\right) \\
& +\mathfrak{l}_{1}(\mathbf{p}) \mathbb{R}_{1}+\mathfrak{l}_{2}(\mathbf{p}) \mathbb{R}_{2}+\mathfrak{l}_{3}(\mathbf{p})\left(\mathbb{R}_{3}+\mathbb{R}_{4}\right)+\mathfrak{l}_{4}(\mathbf{p})\left(\mathbb{R}_{5}+\mathbb{R}_{6}\right)
\end{aligned}
$$

where $\mathfrak{l}_{i}(\cdot), i=1,2,3$ are as defined in Eq. (102) and $\mathfrak{l}_{4}(\mathbf{p})=s \ell(\mathbf{p})$.

6.6 Rhombic, Monoclinic and Triclinic Systems

There are no practical analytical results for the exponential, logarithm and square root for these low symmetries. The purely numerical route is recommended.

\section{The Closest Tensors Using Logarithmic Norms}

We now consider the problem of finding the closest elasticity tensors using the Riemannian and the logarithmic distance functions. The solutions use the machinery developed in the previous Section for evaluating functions of tensors. We begin with the higher symmetries.

\subsection{The Closest Isotropic Tensor Using the Riemannian Norm}

We want to find the closest isotropic tensor $\mathbb{C}_{R}^{\text {iso }}=3 \kappa_{R} \mathbb{J}+2 \mu_{R} \mathbb{K}$ to a given elasticity tensor $\mathbb{C}$, i.e., find $\kappa_{R}>0$ and $\mu_{R}>0$ such that the Riemannian distance $\mathrm{d}_{R}\left(\mathbb{C}, \mathbb{C}_{R}^{\text {iso }}\right)$ is minimized, where $d_{R}$ is defined in Eq. (11c). To find the optimality conditions, we note that minimizing the Riemannian distance is equivalent to minimizing the square of this distance, and recall the following result [24, Prop. 2.1]

$$
\frac{d}{d t} \operatorname{tr}\left[\log ^{2} \mathbf{X}(t)\right]=2 \operatorname{tr}\left[\log \mathbf{X}(t) \mathbf{X}^{-1}(t) \frac{d}{d t} \mathbf{X}(t)\right] .
$$


Here $\mathbf{X}(t)$ is a real matrix-valued function of the real variable $t$ such that, for all $t$ in its domain, $\mathbf{X}(t)$ is an invertible matrix which does not have eigenvalues on the closed negative real line. This result applies to fourth-order elasticity tensors via the isomorphism (7).

Using this general result implies two conditions,

$$
\begin{aligned}
& \frac{\partial \mathrm{d}_{R}^{2}}{\partial \kappa_{R}}\left(\mathbb{C}, \mathbb{C}_{R}^{\text {iso }}\right)=\frac{2}{\kappa_{R}} \operatorname{tr}\left[\log \left(\mathbb{C}^{-1} \mathbb{C}_{R}^{\text {iso }}\right) \mathbb{J}\right], \\
& \frac{\partial \mathrm{d}_{R}^{2}}{\partial \mu_{R}}\left(\mathbb{C}, \mathbb{C}_{R}^{\text {iso }}\right)=\frac{2}{\mu_{R}} \operatorname{tr}\left[\log \left(\mathbb{C}^{-1} \mathbb{C}_{R}^{\text {iso }}\right) \mathbb{K}\right] .
\end{aligned}
$$

Hence, the optimality conditions for $\mathbb{C}_{R}^{\text {iso }}$ are

$$
\operatorname{tr}\left[\log \left(\mathbb{C}^{-1} \mathbb{C}_{R}^{\text {iso }}\right) \mathbb{J}\right]=0, \quad \operatorname{tr}\left[\log \left(\mathbb{C}^{-1} \mathbb{C}_{R}^{\text {iso }}\right) \mathbb{K}\right]=0 .
$$

In view of the partition (20), addition of these two equations yields

$$
\operatorname{tr}\left[\log \left(\mathbb{C}^{-1} \mathbb{C}_{R}^{\text {iso }}\right)\right]=0,
$$

which can be rewritten as ${ }^{3}$ (see Appendix B)

$$
\operatorname{det}\left(\mathbb{C}^{-1} \mathbb{C}_{R}^{\text {iso }}\right)=1,
$$

or, equivalently,

$$
\operatorname{det} \mathbb{C}=\operatorname{det} \mathbb{C}_{R}^{\text {iso }} \text {. }
$$

This is the counterpart of the fact that, for the Euclidean distance, the closest isotropic tensor has the same trace as the given elasticity tensor.

\subsubsection{The Closest Isotropic Tensor to a Given Cubic Tensor}

For $\mathbb{C}=a \mathbb{J}+b \mathbb{L}+c \mathbb{M}$, the conditions (115) 1 and (116), after some algebra, reduce to

$$
\ln \frac{3 \kappa_{R}}{a}=0, \quad a b^{3} c^{2}=3 \kappa_{R}\left(2 \mu_{R}\right)^{5},
$$

which can readily be solved to yield

$$
3 \kappa_{R}=a, \quad 2 \mu_{R}=\left(b^{3} c^{2}\right)^{1 / 5} .
$$

\subsubsection{The Closest Isotropic Tensor to a Given Transversely Isotropic Tensor}

For $\mathbb{C}=a \mathbb{E}_{1}+b \mathbb{E}_{2}+c\left(\mathbb{E}_{3}+\mathbb{E}_{4}\right)+f \mathbb{F}+g \mathbb{G}$, the conditions (115) 1 and (116) yield

$$
3 \ln \delta(\mathbf{x})-\ell(\mathbf{x})(\beta(\mathbf{x})-2 \sqrt{2} z)=0, \quad\left(a b-c^{2}\right) f^{2} g^{2}=3 \kappa_{R}\left(2 \mu_{R}\right)^{5},
$$

3 Here and throughout, the determinant of a fourth-order elasticity tensor $\mathbb{C}$ is $\operatorname{det} \mathbb{C}=\prod_{I=1}^{6} \Lambda_{I}$, where the $\Lambda_{I}$ 's are the eigenvalues of $\mathbb{C}$. From Eq. (8) we have $\operatorname{det} \mathbb{C}=\operatorname{det} \widehat{\mathbf{C}}$, where $\widehat{\mathbf{C}}$ is the associated six-dimensional second-order tensor.

空 Springer 
where $\mathbf{x}=(x, y, z, 0)$ with

$$
\left(\begin{array}{ll}
x & z \\
z & y
\end{array}\right)=\boldsymbol{\Sigma}^{t} \boldsymbol{\Lambda}\left(\begin{array}{ll}
A & C \\
C & B
\end{array}\right) \boldsymbol{\Lambda} \boldsymbol{\Sigma}, \quad\left(\begin{array}{ll}
A & C \\
C & B
\end{array}\right)=\boldsymbol{\Sigma}\left(\begin{array}{ll}
a & c \\
c & b
\end{array}\right) \boldsymbol{\Sigma}^{t},
$$

where

$$
\boldsymbol{\Sigma}=\frac{1}{\sqrt{3}}\left(\begin{array}{cc}
1 & \sqrt{2} \\
-\sqrt{2} & 1
\end{array}\right), \quad \boldsymbol{\Lambda}=\operatorname{diag}\left(\frac{1}{\sqrt{3 \kappa_{R}}}, \frac{1}{\sqrt{2 \mu_{R}}}\right)
$$

Since $\boldsymbol{\Sigma}$ is an orthogonal transformation, it follows that $A B-C^{2}=a b-c^{2}$, $A+B=a+b, x y-z^{2}=\left(a b-c^{2}\right) /\left(6 \kappa_{R} \mu_{R}\right)$ and $x+y=(a+b) /\left(6 \kappa_{R} \mu_{R}\right)$.

Equation $(118)_{2}$ can be used to eliminate $\kappa_{R}$ in Eq. (118) $)_{1}$ and hence we obtain a single nonlinear equation for a single unknown. An appropriate choice for this unknown is the positive variable $\xi$ defined by the ratio

$$
\xi^{2}=\frac{3 \kappa_{R}}{2 \mu_{R}}=\frac{1+v_{R}}{1-2 v_{R}}
$$

where $v_{R}$ is the Poisson's ratio of the closest isotropic tensor with respect to the Riemannian distance. Then Eq. (118) yields the following equation for $\xi$

$$
\left(\bar{A}-\bar{B} \xi^{2}\right) \ln \frac{\bar{A}+\bar{B} \xi^{2}+R(\xi)}{\bar{A}+\bar{B} \xi^{2}-R(\xi)}-R(\xi) \ln \frac{\xi^{4 / 3}}{\bar{A} \bar{B}-\bar{C}^{2}}=0,
$$

where

$$
R(\xi)=\sqrt{\left(\bar{A}-\bar{B} \xi^{2}\right)^{2}+4 \bar{C}^{2} \xi^{2}}
$$

and $\bar{A}=A /(\operatorname{det} \mathbb{C})^{1 / 6}, \bar{B}=B /(\operatorname{det} \mathbb{C})^{1 / 6}, \bar{C}=C /(\operatorname{det} \mathbb{C})^{1 / 6}$. Note here that for this type of symmetry det $\mathbb{C}=\left(a b-c^{2}\right) f^{2} g^{2}$.

The isotropic moduli therefore depend upon three unidimensional combinations of the transversely isotropic moduli, $\bar{A}, \bar{B}$ and $\bar{C}$. Once the solution $\xi$ of Eq. (121) is found, the isotropic moduli are given by

$$
3 \kappa_{R}=(\operatorname{det} \mathbb{C})^{1 / 6} \xi^{5 / 3}, \quad 2 \mu_{R}=(\operatorname{det} \mathbb{C})^{1 / 6} \xi^{-1 / 3} .
$$

\subsubsection{The Closest Isotropic Tensor to a Given Tetragonal Tensor}

For $\mathbb{C}=a \mathbb{E}_{1}+b \mathbb{E}_{2}+c\left(\mathbb{E}_{3}+\mathbb{E}_{4}\right)+p \mathbb{F}_{1}+q \mathbb{F}_{2}+r\left(\mathbb{F}_{3}+\mathbb{F}_{4}\right)+g \mathbb{G}$, the conditions $(115)_{1}$ and (116) yield

$$
3 \ln \delta(\mathbf{x})-\ell(\mathbf{x})(\beta(\mathbf{x})-2 \sqrt{2} z)=0, \quad\left(a b-c^{2}\right)\left(p q-r^{2}\right) g^{2}=3 \kappa_{R}\left(2 \mu_{R}\right)^{5},
$$

where again $\mathbf{x}=(x, y, z, 0)$ is defined by Eq. (119). The solution is obtained in similar manner to that for transverse isotropy, thus, $\kappa_{R}$ and $\mu_{R}$ are given by Eq. (122) but in this case det $\mathbb{C}=\left(a b-c^{2}\right)\left(p q-r^{2}\right) g^{2}$. 


\subsubsection{The Closest Isotropic Tensor to a Given Trigonal Tensor}

For $\mathbb{C}=a \mathbb{E}_{1}+b \mathbb{E}_{2}+c\left(\mathbb{E}_{3}+\mathbb{E}_{4}\right)+p \mathbb{R}_{1}+q \mathbb{R}_{2}+r\left(\mathbb{R}_{3}+\mathbb{R}_{4}\right)+s\left(\mathbb{R}_{5}+\mathbb{R}_{6}\right)$, the conditions $(115)_{1}$ and (116) yield

$$
3 \ln \delta(\mathbf{x})-\ell(\mathbf{x})(\beta(\mathbf{x})-2 \sqrt{2} z)=0, \quad \frac{1}{2}\left(a b-c^{2}\right)\left(p q-r^{2}-s^{2}\right)^{2}=3 \kappa_{R}\left(2 \mu_{R}\right)^{5},
$$

with $\mathbf{x}=(x, y, z, 0)$ of Eq. (119). The isotropic elastic moduli are obtained in similar fashion as for of transverse isotropy, i.e., $\kappa_{R}$ and $\mu_{R}$ are given by Eq. (122) but now $\operatorname{det} \mathbb{C}=\frac{1}{2}\left(a b-c^{2}\right)\left(p q-r^{2}-s^{2}\right)^{2}$.

\subsection{The Closest Cubic Tensor Using the Riemannian Norm}

The optimality conditions that define the closest cubic tensor $\mathbb{C}_{R}^{\text {cub }}=3 \kappa_{R} \mathbb{J}+2 \mu_{R} \mathbb{L}+$ $2 \eta_{R} \mathbb{M}$ to a given elasticity tensor $\mathbb{C}$ of lower symmetry, are obtained by minimizing the Riemannian distance $\mathrm{d}_{R}\left(\mathbb{C}, \mathbb{C}_{R}^{\text {cub }}\right)$ :

$$
\begin{aligned}
& \frac{\partial \mathrm{d}_{R}^{2}}{\partial \kappa_{R}}\left(\mathbb{C}, \mathbb{C}_{R}^{\mathrm{cub}}\right)=\frac{2}{\kappa_{R}} \operatorname{tr}\left[\log \left(\mathbb{C}^{-1} \mathbb{C}_{R}^{\mathrm{cub}}\right) \mathbb{J}\right]=0, \\
& \frac{\partial \mathrm{d}_{R}^{2}}{\partial \mu_{R}}\left(\mathbb{C}, \mathbb{C}_{R}^{\mathrm{cub}}\right)=\frac{2}{\mu_{R}} \operatorname{tr}\left[\log \left(\mathbb{C}^{-1} \mathbb{C}_{R}^{\mathrm{cub}}\right) \mathbb{L}\right]=0, \\
& \frac{\partial \mathrm{d}_{R}^{2}}{\partial \eta_{R}}\left(\mathbb{C}, \mathbb{C}_{R}^{\mathrm{cub}}\right)=\frac{2}{\eta_{R}} \operatorname{tr}\left[\log \left(\mathbb{C}^{-1} \mathbb{C}_{R}^{\mathrm{cub}}\right) \mathbb{M}\right]=0 .
\end{aligned}
$$

As $\kappa_{R}>0, \mu_{R}>0$ and $\eta_{R}>0$, these conditions are equivalent to

$$
\operatorname{tr}\left[\log \left(\mathbb{C}^{-1} \mathbb{C}_{R}^{\mathrm{cub}}\right) \mathbb{V}\right]=0, \quad \mathbb{V}=\mathbb{J}, \mathbb{L}, \mathbb{M}
$$

In view of the partition $(26)_{1}$, combining these three equations yields, in the same manner as for Eq. (115),

$$
\operatorname{det} \mathbb{C}=\operatorname{det} \mathbb{C}_{R}^{\mathrm{cub}}=3 \kappa_{R}\left(2 \mu_{R}\right)^{3}\left(2 \eta_{R}\right)^{2} .
$$

If $\mathbb{C}=a \mathbb{E}_{1}+b \mathbb{E}_{2}+c\left(\mathbb{E}_{3}+\mathbb{E}_{4}\right)+p \mathbb{F}_{1}+q \mathbb{F}_{2}+r\left(\mathbb{F}_{3}+\mathbb{F}_{4}\right)+g \mathbb{G}$, i.e., is of tetragonal symmetry, then (126) $\mathbb{V}=\mathbb{J}$ reduces to

$$
3 \ln \delta(\tilde{\mathbf{x}})-\ell(\tilde{\mathbf{x}})(\beta(\tilde{\mathbf{x}})-2 \sqrt{2} \tilde{z})=0
$$

where $\tilde{\mathbf{x}}=(\tilde{x}, \tilde{y}, \tilde{z}, 0)$ with

$$
\left(\begin{array}{ll}
\tilde{x} & \tilde{z} \\
\tilde{z} & \tilde{y}
\end{array}\right)=\Sigma^{t}\left(\begin{array}{ll}
\tilde{A} & \tilde{C} \\
\tilde{C} & \tilde{B}
\end{array}\right) \Sigma=\Sigma^{t} \tilde{\Lambda} \Sigma\left(\begin{array}{ll}
a & c \\
c & b
\end{array}\right) \Sigma^{t} \tilde{\Lambda} \Sigma,
$$

where $\boldsymbol{\Sigma}$ is as defined in Eq. (120) and

$$
\tilde{\boldsymbol{\Lambda}}=\operatorname{diag}\left(\frac{1}{\sqrt{3 \kappa_{R}}}, \frac{1}{\sqrt{2 \eta_{R}}}\right) .
$$


Again, since $\Sigma$ is an orthogonal transformation, it follows that $\tilde{A} \tilde{B}-\tilde{C}^{2}=a b-c^{2}$, $\tilde{A}+\tilde{B}=a+b, \tilde{x} \tilde{y}-\tilde{z}^{2}=\left(a b-c^{2}\right) /\left(6 \kappa_{R} \eta_{R}\right)$, and $\tilde{x}+\tilde{y}=(a+b) /\left(6 \kappa_{R} \eta_{R}\right)$. Eq. (127) becomes

$$
\left(a b-c^{2}\right)\left(p q-r^{2}\right) g^{2}=3 \kappa_{R}\left(2 \mu_{R}\right)^{3}(2 \eta)^{2} .
$$

Let $\zeta$ and $\sigma$ be the nondimensional positive variables defined by

$$
\zeta^{2}=\frac{3 \kappa_{R}}{2 \eta_{R}}, \quad \sigma^{2}=\frac{\mu_{R}}{\eta_{R}}
$$

then Eqs. (128) and (126) $\mathbb{V}=\mathbb{L}$ yield the following coupled two equations for $\zeta$ and $\sigma$

$$
\begin{aligned}
& \left(\tilde{A}-\tilde{B} \zeta^{2}\right) \ln \frac{\tilde{A}+\tilde{B} \zeta^{2}+Q_{1}(\zeta)}{\tilde{A}+\tilde{B} \zeta^{2}-Q_{1}(\zeta)}-Q_{1}(\zeta) \ln \frac{\zeta^{4 / 3} \sigma^{-2}(\operatorname{det} \mathbb{C})^{1 / 3}}{a b-c^{2}}=0, \\
& \left(p-q \sigma^{2}\right) \ln \frac{p+q \sigma^{2}+Q_{2}(\sigma)}{p+q \sigma^{2}-Q_{2}(\sigma)}-Q_{2}(\sigma) \ln \frac{\zeta^{-2} \sigma^{-1}\left(a b-c^{2}\right)}{g^{2}}=0 .
\end{aligned}
$$

where

$$
Q_{1}(\zeta)=\sqrt{\left(\tilde{A}-\tilde{B} \zeta^{2}\right)^{2}+4 \tilde{C}^{2} \zeta^{2}}, \quad Q_{2}(\sigma)=\sqrt{(p-q \sigma)^{2}+4 r^{2} \sigma^{2}}
$$

The three moduli of the closest cubic elasticity tensor are then given by

$$
3 \kappa_{R}=\zeta^{5 / 3} \sigma^{-1}(\operatorname{det} \mathbb{C})^{1 / 6}, \quad 2 \mu_{R}=\zeta^{-1 / 3} \sigma(\operatorname{det} \mathbb{C})^{1 / 6}, \quad 2 \eta_{R}=\zeta^{-1 / 3} \sigma^{-1}(\operatorname{det} \mathbb{C})^{1 / 6} .
$$

\subsection{The Closest Transversely Isotropic Tensor Using the Riemannian Norm}

We want to find the closest, in the Riemannian metric, transversely isotropic tensor $\mathbb{C}_{R}^{\text {hex }}=a \mathbb{E}_{1}+b \mathbb{E}_{2}+c\left(\mathbb{E}_{3}+\mathbb{E}_{4}\right)+f \mathbb{F}+g \mathbb{G}$ to a given elasticity tensor $\mathbb{C}$ of lower symmetry, i.e., find $a>0, b>0 f>0, g>0$ and $c$ with $a b-c^{2}>0$ such that the Riemannian distance $\mathrm{d}_{R}\left(\mathbb{C}, \mathbb{C}_{R}^{\text {hex }}\right)$ is minimized.

The optimality conditions are

$$
\begin{aligned}
& \frac{\partial \mathrm{d}_{R}^{2}}{\partial a}\left(\mathbb{C}, \mathbb{C}_{R}^{\text {hex }}\right)=\frac{2}{a b-c^{2}} \operatorname{tr}\left[\log \left(\mathbb{C}^{-1} \mathbb{C}_{R}^{\text {hex }}\right)\left(b \mathbb{E}_{1}-c \mathbb{E}_{4}\right)\right]=0, \\
& \frac{\partial \mathrm{d}_{R}^{2}}{\partial b}\left(\mathbb{C}, \mathbb{C}_{R}^{\text {hex }}\right)=\frac{2}{a b-c^{2}} \operatorname{tr}\left[\log \left(\mathbb{C}^{-1} \mathbb{C}_{R}^{\text {hex }}\right)\left(a \mathbb{E}_{2}-c \mathbb{E}_{3}\right)\right]=0, \\
& \frac{\partial \mathrm{d}_{R}^{2}}{\partial c}\left(\mathbb{C}, \mathbb{C}_{R}^{\text {hex }}\right)=\frac{2}{a b-c^{2}} \operatorname{tr}\left[\log \left(\mathbb{C}^{-1} \mathbb{C}_{R}^{\text {hex }}\right)\left(-c \mathbb{E}_{1}-c \mathbb{E}_{2}+b \mathbb{E}_{3}+a \mathbb{E}_{4}\right)\right]=0, \\
& \frac{\partial \mathrm{d}_{R}^{2}}{\partial f}\left(\mathbb{C}, \mathbb{C}_{R}^{\text {hex }}\right)=\frac{2}{f} \operatorname{tr}\left[\log \left(\mathbb{C}^{-1} \mathbb{C}_{R}^{\text {hex }}\right) \mathbb{F}\right]=0, \\
& \frac{\partial \mathrm{d}_{R}^{2}}{\partial g}\left(\mathbb{C}, \mathbb{C}_{R}^{\text {hex }}\right)=\frac{2}{g} \operatorname{tr}\left[\log \left(\mathbb{C}^{-1} \mathbb{C}_{R}^{\text {hex }}\right) \mathbb{G}\right]=0 .
\end{aligned}
$$


As $a>0, b>0, a b-c^{2}>0, f>0$ and $g>0$, these five conditions are equivalent to

$$
\operatorname{tr}\left[\log \left(\mathbb{C}^{-1} \mathbb{C}_{R}^{\mathrm{hex}}\right) \mathbb{V}\right]=0, \quad \mathbb{V}=b \mathbb{E}_{1}-c \mathbb{E}_{4}, a \mathbb{E}_{2}-c \mathbb{E}_{3}, \mathbb{E}_{1}+\mathbb{E}_{2}, \mathbb{F}, \mathbb{G}
$$

Combining the conditions for $\mathbb{E}_{1}+\mathbb{E}_{2}, \mathbb{F}$ and $\mathbb{G}$, and partition (32) implies the constraint

$$
\operatorname{det} \mathbb{C}=\operatorname{det} \mathbb{C}_{R}^{\text {hex }} \text {. }
$$

\subsection{The Closest Tensors Using the Log-Euclidean Norm}

The closest elasticity tensors according to the log-Euclidean metric follow by minimizing $\mathrm{d}_{L}^{2}\left(\mathbb{C}, \mathbb{C}_{\text {sym }}\right)$. The stationarity condition implies that $\left(\log \mathbb{C}-\log \mathbb{C}_{\text {sym }}\right)$ is orthogonal (in the Euclidean sense) to the symmetry class, and hence

$$
\log \mathbb{C}_{\text {sym }}=P_{\text {sym }} \log \mathbb{C},
$$

where the Euclidean projector is defined in Eq. (67). We may therefore write

$$
\mathbb{C}_{\text {sym }}=\exp \left(P_{\text {sym }} \log \mathbb{C}\right) \text {. }
$$

This may be evaluated for the particular symmetries using the explicit expressions for $P_{\text {sym }}$ in Eq. (68), combined with the formulas for the logarithm and exponential of elasticity tensors in Section 6. We note in particular the Euclidean property,

$$
\mathrm{d}_{L}^{2}\left(\mathbb{C}, \mathbb{C}_{\text {sym A }}\right)=\mathrm{d}_{L}^{2}\left(\mathbb{C}, \mathbb{C}_{\text {sym B }}\right)+\mathrm{d}_{L}^{2}\left(\mathbb{C}_{\text {sym B }}, \mathbb{C}_{\text {sym A }}\right), \quad \operatorname{sym~A} \geq \operatorname{sym~B.}
$$

We note the following identity, which is a consequence of the requirement that $\left(\log \mathbb{C}-\log \mathbb{C}^{\text {sym }}\right)$ is orthogonal to each of the basis tensors,

$$
\operatorname{tr}\left[\mathbb{V}_{i} \log \mathbb{C}\right]=\operatorname{tr}\left[\mathbb{V}_{i} \log \mathbb{C}_{\text {sym }}\right] .
$$

In the remainder of this section we apply this to the particular cases of isotropy and cubic isotropy, for which we derive explicit formulas for the closest moduli.

\subsubsection{The Closest Isotropic Tensor Using the Log-Euclidean Norm}

There are two basis tensors and therefore the general conditions (137) become, using Eq. (83) with $\mathbb{C}_{L}^{\text {iso }}=3 \kappa_{L} \mathbb{J}+2 \mu_{L} \mathbb{K}$,

$$
\ln 3 \kappa_{L}=\operatorname{tr}[\mathbb{J} \log \mathbb{C}], \quad \ln 2 \mu_{L}=\frac{1}{5} \operatorname{tr}[\mathbb{K} \log \mathbb{C}] .
$$

Hence, we obtain explicit formulas

$$
3 \kappa_{L}=\exp (\operatorname{tr}[\mathbb{J} \log \mathbb{C}]), \quad 2 \mu_{L}=\exp \left(\frac{1}{5} \operatorname{tr}[\mathbb{K} \log \mathbb{C}]\right) .
$$

Using $\mathbb{I}=\mathbb{J}+\mathbb{K}$ and Eq. (B.14), it follows that

$$
3 \kappa_{L}\left(2 \mu_{L}\right)^{5}=\operatorname{det} \mathbb{C} .
$$




\subsubsection{The Closest Isotropic Tensor to a Given Cubic Tensor}

For $\mathbb{C}=a \mathbb{J}+b \mathbb{L}+c \mathbb{M}$, we use Eq. (88) and $\mathbb{K}=\mathbb{L}+\mathbb{M}$ to simplify the conditions (138). After some algebra we find [17]

$$
3 \kappa_{L}=a, \quad 2 \mu_{L}=\left(b^{3} c^{2}\right)^{1 / 5} .
$$

These moduli coincide with those obtained for the Riemannian norm. This is a consequence of the fact that when two tensors $\mathbb{C}_{1}$ and $\mathbb{C}_{2}$ commute under multiplication we have $\log \left(\mathbb{C}_{1} \mathbb{C}_{2}^{-1}\right)=\log \mathbb{C}_{1}-\log \mathbb{C}_{2}$.

\subsubsection{The Closest Isotropic Tensor to a Given Transversely Isotropic Tensor}

For $\mathbb{C}=a \mathbb{E}_{1}+b \mathbb{E}_{2}+c\left(\mathbb{E}_{3}+\mathbb{E}_{4}\right)+f \mathbb{F}+g \mathbb{G}$, we use Eqs. (101), (138) $)_{1}$ and $(\mathrm{A} .22)_{1}$ to get

$$
\ln 3 \kappa_{L}=\frac{1}{2} \ln \left(a b-c^{2}\right)+\frac{b-a+4 \sqrt{2} c}{12 \gamma(\mathbf{a})} \ln \frac{a+b+2 \gamma(\mathbf{a})}{a+b-2 \gamma(\mathbf{a})} .
$$

Thus,

$$
3 \kappa_{L}=\sqrt{a b-c^{2}}\left(\frac{a+b+\sqrt{(a-b)^{2}+4 c^{2}}}{a+b-\sqrt{(a-b)^{2}+4 c^{2}}}\right)^{\frac{b-a+4 \sqrt{2} c}{6 \sqrt{(a-b)^{2}+4 c^{2}}}},
$$

and the shear modulus then follows from Eq. (140) as

$$
2 \mu_{L}=\left[\frac{1}{3 \kappa_{L}}\left(a b-c^{2}\right) f^{2} g^{2}\right]^{1 / 5} .
$$

Note that the Eq. (141) for $\kappa_{L}$ can be cast in the form of Eq. (122) 1 , where $\xi$ satisfies an equation similar to Eq. (121),

$$
(\bar{A}-\bar{B}) \ln \frac{\bar{A}+\bar{B}+R(1)}{\bar{A}+\bar{B}-R(1)}-R(1) \ln \frac{\xi^{10 / 3}}{\bar{A} \bar{B}-\bar{C}^{2}}=0 .
$$

\subsubsection{The Closest Isotropic Tensor to a Given Tetragonal Tensor}

For $\mathbb{C}=a \mathbb{E}_{1}+b \mathbb{E}_{2}+c\left(\mathbb{E}_{3}+\mathbb{E}_{4}\right)+p \mathbb{F}_{1}+q \mathbb{F}_{2}+r\left(\mathbb{F}_{3}+\mathbb{F}_{4}\right)+g \mathbb{G}$, we again use Eqs. (101), (138) $)_{1}$ and the identity (A.22) $)_{1}$ to derive Eq. (142) for $\kappa_{L}$. The shear modulus is

$$
2 \mu_{L}=\left[\frac{1}{3 \kappa_{L}}\left(a b-c^{2}\right)\left(p q-r^{2}\right) g^{2}\right]^{1 / 5} .
$$

\subsubsection{The Closest Isotropic Tensor to a Given Trigonal Tensor}

For $\mathbb{C}=a \mathbb{E}_{1}+b \mathbb{E}_{2}+c\left(\mathbb{E}_{3}+\mathbb{E}_{4}\right)+p \mathbb{R}_{1}+q \mathbb{R}_{2}+r\left(\mathbb{R}_{3}+\mathbb{R}_{4}\right)+s\left(\mathbb{R}_{5}+\mathbb{R}_{6}\right)$, the condition (142) for $\kappa_{L}$ is again recovered, while $\mu_{L}$ follows from

$$
2 \mu_{L}=\left[\frac{1}{3 \kappa_{L}}\left(a b-c^{2}\right)\left(p q-r^{2}-s^{2}\right)^{2}\right]^{1 / 5} .
$$




\subsubsection{The Closest Cubic Tensor Using the Log-Euclidean Norm}

Proceeding in the same way as for the isotropic case, in this case with three basis tensors, $\mathbb{C}_{L}^{\text {cub }}=3 \kappa_{L} \mathbb{J}+2 \mu_{L} \mathbb{L}+2 \eta_{L} \mathbb{M}$, we find that the closest cubic tensor has explicit solution

$$
\begin{aligned}
3 \kappa_{L} & =\exp (\operatorname{tr}[\mathbb{J} \log \mathbb{C}]), \\
2 \mu_{L} & =\exp \left(\frac{1}{3} \operatorname{tr}[\mathbb{L} \log \mathbb{C}]\right) \\
2 \eta_{L} & =\exp \left(\frac{1}{2} \operatorname{tr}[\mathbb{M} \log \mathbb{C}]\right) .
\end{aligned}
$$

The moduli satisfy the same determinant constraint as for the Riemannian norm, in this case

$$
3 \kappa_{L}\left(2 \mu_{L}\right)^{3}\left(2 \eta_{L}\right)^{2}=\operatorname{det} \mathbb{C}
$$

\subsubsection{The Closest Cubic Tensor to a Given Tetragonal Tensor}

For $\mathbb{C}=a \mathbb{E}_{1}+b \mathbb{E}_{2}+c\left(\mathbb{E}_{3}+\mathbb{E}_{4}\right)+p \mathbb{F}_{1}+q \mathbb{F}_{2}+r\left(\mathbb{F}_{3}+\mathbb{F}_{4}\right)+g \mathbb{G}$, the bulk modulus $\kappa_{L}$ is again given by Eq. (142). We use

$$
\mathbb{L}=\mathbb{F}_{1}+\mathbb{G},
$$

to obtain

$$
2 \mu_{L}=\left(p q-r^{2}\right)^{1 / 6} g^{2 / 3}\left(\frac{p+q+\sqrt{(p-q)^{2}+4 r^{2}}}{p+q-\sqrt{(p-q)^{2}+4 r^{2}}}\right)^{\frac{p-q}{2 \sqrt{(p-q)^{2}+4 r^{2}}}} .
$$

The shear modulus $\eta_{L}$ follows from

$$
2 \eta_{L}=\left[\frac{\left(a b-c^{2}\right)\left(p q-r^{2}\right) g^{2}}{3 \kappa_{L}\left(2 \mu_{L}\right)^{3}}\right]^{1 / 2} .
$$

\subsubsection{The Closest Transversely Isotropic Tensor Using the Log-Euclidean Norm}

In this case there are five basis tensors, $\mathbb{C}_{L}^{\text {hex }}=a \mathbb{E}_{1}+b \mathbb{E}_{2}+c\left(\mathbb{E}_{3}+\mathbb{E}_{4}\right)+f \mathbb{F}+$ $g \mathbb{G}$. Using Eqs. (101) and (137), we have

$$
f=\exp \left(\frac{1}{2} \operatorname{tr}[\mathbb{F} \log \mathbb{C}]\right), \quad g=\exp \left(\frac{1}{2} \operatorname{tr}[\mathbb{G} \log \mathbb{C}]\right),
$$

and the remaining three moduli follow from the identities

$$
\mathfrak{l}_{1}(\mathbf{a})=\operatorname{tr}\left[\mathbb{E}_{1} \log \mathbb{C}\right], \mathfrak{l}_{2}(\mathbf{a})=\operatorname{tr}\left[\mathbb{E}_{2} \log \mathbb{C}\right], \mathfrak{l}_{3}(\mathbf{a})=\frac{1}{2} \operatorname{tr}\left[\left(\mathbb{E}_{3}+\mathbb{E}_{4}\right) \log \mathbb{C}\right] .
$$


Thus,

$$
\begin{cases}a=\delta \frac{\cosh (\theta+\phi \cosh \theta)}{\cosh \theta}, b=\delta \frac{\cosh (\theta-\phi \cosh \theta)}{\cosh \theta}, c=\delta \frac{\sinh (\phi \cosh \theta)}{\cosh \theta}, & \text { if } \phi \neq 0, \\ a=\delta e^{\psi}, \quad b=\delta e^{-\psi}, \quad c=0, & \text { if } \phi=0,\end{cases}
$$

where

$$
\begin{array}{lll}
\phi=\frac{1}{2} \operatorname{tr}\left[\left(\mathbb{E}_{3}+\mathbb{E}_{4}\right) \log \mathbb{C}\right], & \psi=\frac{1}{2} \operatorname{tr}\left[\left(\mathbb{E}_{1}-\mathbb{E}_{2}\right) \log \mathbb{C}\right], \\
\delta=\exp \left(\frac{1}{2} \operatorname{tr}\left[\left(\mathbb{E}_{1}+\mathbb{E}_{2}\right) \log \mathbb{C}\right]\right), & \theta=\log \left[\frac{\psi}{\phi}+\left(\frac{\psi^{2}}{\phi^{2}}+1\right)^{1 / 2}\right] .
\end{array}
$$

\section{Application and Numerical Examples}

We illustrate the methods developed above by considering an example of a general elasticity tensor, with no assumed symmetry. This type of data raises a problem typically encountered, i.e., find the optimal orientation of the symmetry axes in addition to finding the closest elastic tensors for a given symmetry axes or planes. We first present the data, and the isotropic approximations, and then consider the question of orientation.

\subsection{Example}

A complete set of 21 elastic constants were determined ultrasonically by François et al. [7]. The reader is referred to their paper for details of the measurement technique. The raw moduli are

$$
\mathbf{C}=\left(\begin{array}{cccccc}
243 & 136 & 135 & 22 & 52 & -17 \\
136 & 239 & 137 & -28 & 11 & 16 \\
135 & 137 & 233 & 29 & -49 & 3 \\
22 & -28 & 29 & 133 & -10 & -4 \\
52 & 11 & -49 & -10 & 119 & -2 \\
-17 & 16 & 3 & -4 & -2 & 130
\end{array}\right) \quad(\mathrm{GPa})
$$

We first search for the presence of symmetry planes, which are an indicator of underlying symmetry. Following Cowin and Mehrabadi [35], define $\mathbf{A}$ and $\mathbf{B}$ by

$$
A_{i j}=C_{i j k k}, \quad B_{i j}=C_{i k j k} .
$$

If $\mathbf{A}$ and $\mathbf{B}$ have no common eigenvectors then there are no planes of reflection symmetry and the material has no effective elastic symmetry [48]. We find that the two sets of eigenvectors are not coincident, and the smallest angle between any pair from the two sets of eigenvectors is $16^{\circ}$. Materials with symmetry higher than orthorhombic have five or fewer distinct eigenvalues $\Lambda_{I}$ [20]. For the given moduli, we find $\Lambda_{I}=47,79,244,285,312,512$, consistent with the material having no symmetry plane.

We next consider isotropic approximations to the 21 moduli. The Euclidean and log-Euclidean approximations follow from Eqs. (68a) and (139), respectively. The 
numerical procedure for finding the closest, in the Riemannian norm, isotropic tensor $\mathbb{C}_{R}^{\text {iso }}=3 \kappa_{R} \mathbb{J}+2 \mu_{R} \mathbb{K}$ is as follows. Define the function

$$
F\left(3 \kappa_{R}, 2 \mu_{R}\right)=\operatorname{tr}\left[\log \left(\mathbb{C}^{-1}\left(3 \kappa_{R} \mathbb{J}+2 \mu_{R} \mathbb{K}\right)\right) \mathbb{J}\right] .
$$

By the equal determinant rule (116), we have $3 \kappa_{R}\left(2 \mu_{R}\right)^{5}=\operatorname{det} \mathbb{C}$, and therefore we need only solve the equation for one of $\kappa_{R}$ or $\mu_{R}$. In practice, we solve

$$
F\left(3 \kappa_{R},\left(\frac{\operatorname{det} \mathbb{C}}{3 \kappa_{R}}\right)^{1 / 5}\right)=0,
$$

using Newton's method.

The isotropic approximations are

\begin{tabular}{l|cc} 
& $\kappa$ & $\mu$ \\
\hline Euclidean, $\mathbb{C}$ & 170.11 & 96.87 \\
Euclidean, $\mathbb{S}$ & 169.33 & 55.81 \\
log-Euclidean & 169.84 & 75.91 \\
Riemannian & 169.69 & 75.92
\end{tabular}

The Euclidean projection is obviously not invariant under inversion. Another way to see this is to consider the product

$$
\widehat{\mathbf{S}}_{\mathrm{iso}} \widehat{\mathbf{C}}_{\mathrm{iso}} \approx 1.00 \widehat{\mathbf{I}}+0.74 \widehat{\mathbf{K}},
$$

where $\widehat{\mathbf{C}}_{\text {iso }}$ and $\widehat{\mathbf{S}}_{\text {iso }}$ are the isotropic projections for the stiffness $\widehat{\mathbf{C}}$ and compliance $\widehat{\mathbf{S}}=\widehat{\mathbf{C}}^{-1}$, respectively. The product (159) is an isotropic tensor, as expected, but not the identity. The analogous product $\widehat{\mathbf{S}}_{\text {sym }} \widehat{\mathbf{C}}_{\text {sym }}$ of the Euclidean stiffness and compliance projections becomes closer to the identity as the symmetry is reduced, although not uniformly. As one measure, we note that the parameter $\operatorname{det}\left(\widehat{\mathbf{S}}_{\mathrm{sym}} \widehat{\mathbf{C}}_{\mathrm{sym}}\right)$, which is unity for the full triclinic matrices, takes the values 4.2, 4.6, 6.6, 4.5, 8.1, 5.3, 15.8 for mon, ort, trig, tet, hex, cub and iso, respectively.

\subsection{Orientation Effects}

This set of moduli present the general problem of finding the optimal orientation of the symmetry axes, for given symmetries. This can be done by a "brute force" approach of searching over all possible orientations of the basis vectors. In practice this is achieved using Euler angles $\left(\theta_{1}, \theta_{2}, \theta_{3}\right)$ to transform from $\left\{\mathbf{e}_{1}, \mathbf{e}_{2}, \mathbf{e}_{3}\right\} \rightarrow$ $\left\{\mathbf{e}_{1}^{\prime}, \mathbf{e}_{2}^{\prime}, \mathbf{e}_{3}^{\prime}\right\}$ by first rotating about the $\mathbf{e}_{3}$ axis by $\theta_{1}$, then about the intermediate $\mathbf{e}_{1}^{\prime \prime}$ axis by $\theta_{2}$, and finally about the $\mathbf{e}_{3}^{\prime}$ axis by $\theta_{3}$. The moduli transform as $\mathbf{C} \rightarrow \mathbf{C}^{\prime}$, and for each triple $\left(\theta_{1}, \theta_{2}, \theta_{3}\right)$ the closest elasticity tensors $\mathbb{C}_{\text {sym }}$ of different symmetries are found. Numerically, we fix the symmetries by reference to the original basis, with $\{\mathbf{a}, \mathbf{b}, \mathbf{c}\}=\left\{\mathbf{e}_{1}, \mathbf{e}_{2}, \mathbf{e}_{3}\right\}$.

Let $\mathrm{d}$ be any of the three distance functions, and define

$$
\rho_{\text {sym }}\left(\theta_{1}, \theta_{2}, \theta_{3}\right)=\frac{\mathrm{d}^{2}\left(\mathbb{C}_{\text {sym }}, \mathbb{C}_{\text {iso }}\right)}{\mathrm{d}^{2}\left(\mathbb{C}, \mathbb{C}_{\text {iso }}\right)}
$$

Thus, $0<\rho_{\text {sym }} \leq 1$, with equality only if the rotated $\mathbf{C}^{\prime}$ has symmetry sym. The preliminary analysis above indicates the absence of any symmetry so we expect $\rho_{\text {sym }}$ to be less than unity. We also define

$$
\rho_{\mathrm{sym}}^{*}=\max _{\theta_{1}, \theta_{2}, \theta_{3}} \rho_{\mathrm{sym}}\left(\theta_{1}, \theta_{2}, \theta_{3}\right) .
$$


Table $1 \rho_{\text {sym }}$ calculated using the Euclidean projection of the stiffness $\mathbb{C}$

\begin{tabular}{lllllll}
\hline $\mathrm{d}_{F}, \mathbb{C}$ & $\rho_{\text {cub }}$ & $\rho_{\text {hex }}$ & $\rho_{\text {tet }}$ & $\rho_{\text {ort }}$ & $\rho_{\text {trig }}$ & $\rho_{\text {mon }}$ \\
\hline cub & 0.91 & 0.60 & 0.91 & 0.92 & 0.61 & 0.94 \\
hex & 0.85 & 0.60 & 0.91 & 0.87 & 0.62 & 0.93 \\
tet & 0.01 & 0.45 & 0.92 & 0.88 & 0.47 & 0.94 \\
ort & 0.03 & 0.54 & 0.90 & 0.94 & 0.58 & 0.95 \\
trig & 0.12 & 0.20 & 0.21 & 0.20 & 0.95 & 0.22 \\
mon & 0.02 & 0.07 & 0.35 & 0.81 & 0.08 & 0.98
\end{tabular}

The diagonal elements in italics are the values of $\rho_{\mathrm{sym}}^{*}$ for the symmetries indicated in the left column. The other numbers in each row are the values of $\rho_{\text {sym }}$ evaluated at the same orientation as $\rho_{\mathrm{sym}}^{*}$.

Orientations at which $\rho_{\text {sym }}=\rho_{\text {sym }}^{*}$ are candidates for symmetry axes that best approximate the moduli.

Tables 1 and 2 list the results using the Euclidean projection for the stiffness and compliance, respectively. Table 3 gives the results for the log-Euclidean distance. The results in Tables 1, 2 and 3 were obtained using $60 \times 60 \times 60$ discretized Euler angles. In general, larger values give an indication of the proximity of the symmetry. These numbers taken together suggests that the material is not well approximated by hex, but that cub and certainly tet are reasonable candidates for approximating symmetries. The analogous log-Euclidean computations, given in Table 3, reinforce this view. Also, $\rho_{\text {cub }}^{*}=0.95$ for the Riemannian norm, using the method below. We focus on the cubic approximation for the remainder of this section.

\subsection{Cubic Approximations}

We note some properties of the optimally oriented cubic approximations. First, the decomposition (70) for the Euclidean cubic projection has an interesting implication. Noting that

$$
2 \mathbb{L}-3 \mathbb{M}=5 \mathbb{J}+2 \mathbb{K}-5(\mathbf{a} \otimes \mathbf{a} \otimes \mathbf{a} \otimes \mathbf{a}+\mathbf{b} \otimes \mathbf{b} \otimes \mathbf{b} \otimes \mathbf{b}+\mathbf{c} \otimes \mathbf{c} \otimes \mathbf{c} \otimes \mathbf{c}),
$$

and using Eq. (58), we may write the cubic length

$$
\left\|\mathbb{C}_{\text {cub }}\right\|^{2}=9 \kappa^{2}+20 \mu^{2}+\frac{5}{6}\left(3 \kappa+4 \mu-c_{a a}-c_{b b}-c_{c c}\right)^{2},
$$

Table $2 \rho_{\text {sym }}$ calculated using the Euclidean projection of the compliance $\mathbb{C}^{-1}$

\begin{tabular}{lllllll}
\hline $\mathrm{d}_{F}, \mathbb{S}$ & $\rho_{\text {cub }}$ & $\rho_{\text {hex }}$ & $\rho_{\text {tet }}$ & $\rho_{\text {ort }}$ & $\rho_{\text {trig }}$ & $\rho_{\text {mon }}$ \\
\hline cub & 0.82 & 0.54 & 0.83 & 0.97 & 0.54 & 0.99 \\
hex & 0.18 & 0.71 & 0.90 & 0.83 & 0.72 & 0.97 \\
tet & 0.82 & 0.35 & 0.96 & 0.97 & 0.35 & 0.98 \\
ort & 0.82 & 0.35 & 0.96 & 0.97 & 0.35 & 0.98 \\
trig & 0.21 & 0.32 & 0.33 & 0.42 & 0.84 & 0.43 \\
mon & 0.82 & 0.54 & 0.83 & 0.97 & 0.54 & 0.99 \\
\hline
\end{tabular}

The elements are determined in the same manner as in Table 1. 
Table $3 \rho_{\text {sym }}$ calculated using the log-Euclidean distance function, determined in the same manner as in Table 1

\begin{tabular}{lllllll}
\hline $\mathrm{d}_{L}$ & $\rho_{\text {cub }}$ & $\rho_{\text {hex }}$ & $\rho_{\text {tet }}$ & $\rho_{\text {ort }}$ & $\rho_{\text {trig }}$ & $\rho_{\text {mon }}$ \\
\hline cub & 0.92 & 0.68 & 0.94 & 0.96 & 0.69 & 0.96 \\
hex & 0.87 & 0.69 & 0.93 & 0.92 & 0.70 & 0.96 \\
tet & 0.11 & 0.47 & 0.95 & 0.71 & 0.48 & 0.96 \\
ort & 0.09 & 0.20 & 0.37 & 0.96 & 0.21 & 0.98 \\
trig & 0.26 & 0.43 & 0.43 & 0.44 & 0.94 & 0.47 \\
mon & 0.91 & 0.60 & 0.92 & 0.96 & 0.62 & 0.99 \\
\hline
\end{tabular}

In particular the diagonal elements are $\rho_{\text {sym }}^{*}$.

where $\kappa$ and $\mu$ are Fedorov's isotropic moduli, and $c_{a a}=\langle\mathbb{C}, \mathbf{a} \otimes \mathbf{a} \otimes \mathbf{a} \otimes \mathbf{a}\rangle=$ $C_{i j k l} a_{i} a_{j} a_{k} a_{l}$, etc. Consider $\left\|\mathbb{C}_{\text {cub }}\right\|$ as a function of the orientation of the cube axes. Since $\kappa$ and $\mu$ are isotropic invariants, and $c_{a a}>0, c_{b b}>0, c_{c c}>0$ on account of the positive definite nature of $\mathbb{C}$, it follows that the largest length occurs when $c_{a a}+c_{b b}+c_{c c}$ achieves it smallest value. This implies that the best cubic approximation in the Euclidean sense occurs in the coordinate system with smallest value of $\left(c_{11}^{\prime}+c_{22}^{\prime}+c_{33}^{\prime}\right)$.

The closest cubic material for log-Euclidean distance function is that which minimizes $\mathrm{d}_{L}\left(\mathbb{C}, \mathbb{C}_{\text {cub }}\right)$. Using the Euclidean property, see Eq. (136), that

$$
\mathrm{d}_{L}^{2}\left(\mathbb{C}, \mathbb{C}_{\text {cub }}\right)=\mathrm{d}_{L}^{2}\left(\mathbb{C}, \mathbb{C}_{\text {iso }}\right)-\mathrm{d}_{L}^{2}\left(\mathbb{C}_{\text {cub }}, \mathbb{C}_{\text {iso }}\right),
$$

and the fact that $\mathrm{d}_{L}\left(\mathbb{C}, \mathbb{C}_{\text {iso }}\right)$ is unchanged under rotation, it follows that the optimal $\mathbb{C}_{\text {cub }}$ maximizes $\mathrm{d}_{L}\left(\mathbb{C}_{\text {cub }}, \mathbb{C}_{\text {iso }}\right)$. Let $\mu_{L i}$ denote the isotropic modulus, from Eq. $(139)_{2}$, then since the bulk modulus $\kappa_{L}$ is the same for isotropy and cubic symmetry, it follows that

$$
\mathrm{d}_{L}\left(\mathbb{C}_{\text {cub }}, \mathbb{C}_{\text {iso }}\right)=\sqrt{\frac{15}{2}}\left|\log \frac{\mu_{L}}{\mu_{L i}}\right| .
$$

Therefore, the optimal orientation is that for which $\frac{1}{2}\left(\frac{\mu_{L}}{\mu_{L i}}+\frac{\mu_{L i}}{\mu_{L}}\right)$ achieves its largest value.

Alternatively, if we define the Euclidean cubic approximation for $\mathbb{C}$ as $\mathbb{C}_{F}^{\text {cub }}=$ $3 \kappa_{F} \mathbb{J}+2 \mu_{F} \mathbb{L}+2 \eta_{F} \mathbb{L}$, then $\kappa_{F}=\kappa$ and the optimal $\mu_{F}$ and $\eta_{F}$ maximize $\left|\mu_{F}-\eta_{F}\right|$ subject to the additive constraint $\frac{3}{5} \mu_{F}+\frac{2}{5} \eta_{F}=\mu$, where $\mu$ is the isotropic (Fedorov) shear modulus. By comparison, the optimal log-Euclidean moduli maximize $\left|\log \mu_{L}-\log \eta_{L}\right|$ subject to the constraint $\frac{3}{5} \log \mu_{L}+\frac{2}{5} \log \eta_{L}=\log \mu$.

The closest cubic tensor in the Riemannian norm, $\mathbb{C}_{R}^{\text {cub }}=3 \kappa_{R} \mathbb{J}+2 \mu_{R} \mathbb{L}+2 \eta_{R} \mathbb{M}$, is obtained using a numerical scheme similar to that for the isotropic case. Define the functions

$$
\begin{aligned}
& F_{1}\left(3 \kappa_{R}, 2 \mu_{R}, 2 \eta_{R}\right)=\operatorname{tr}\left[\log \left(\mathbb{C}^{-1}\left(3 \kappa_{R} \mathbb{J}+2 \mu_{R} \mathbb{L}+2 \eta_{R} \mathbb{M}\right)\right) \mathbb{J}\right], \\
& F_{2}\left(3 \kappa_{R}, 2 \mu_{R}, 2 \eta_{R}\right)=\operatorname{tr}\left[\log \left(\mathbb{C}^{-1}\left(3 \kappa_{R} \mathbb{J}+2 \mu_{R} \mathbb{L}+2 \eta_{R} \mathbb{M}\right)\right) \mathbb{L}\right] .
\end{aligned}
$$

Using the condition (127) of equality of determinants we can eliminate one of the three unknowns, and therefore only need to solve two equations for $\kappa_{R}$ and $\mu_{R}$

$$
F_{1}\left(3 \kappa_{R}, 2 \mu_{R},\left(\frac{\operatorname{det} \mathbb{C}}{3 \kappa_{R}\left(2 \mu_{R}\right)^{3}}\right)^{1 / 2}\right)=0, \quad F_{2}\left(3 \kappa_{R}, 2 \mu_{R},\left(\frac{\operatorname{det} \mathbb{C}}{3 \kappa_{R}\left(2 \mu_{R}\right)^{3}}\right)^{1 / 2}\right)=0 .
$$


These equations are solved by the two-dimensional Newton's method.

The numerical search for the optimal cubic approximation indicates that the orientation which yields the maximum $\rho_{\text {cub }}^{*}$ coincides for the three distance functions for this set of moduli. The optimal cubic moduli are as follows

\begin{tabular}{l|ccc} 
& $\kappa$ & $\mu$ & $\eta$ \\
\hline Eucl, $\mathbb{C}$ & 170.1 & 139.7 & 32.6 \\
Eucl, $\mathbb{S}$ & 169.3 & 135.1 & 29.7 \\
$\log -$ Eucl & 169.7 & 137.5 & 31.2 \\
Riemannian & 169.8 & 138.1 & 30.9
\end{tabular}

The Euclidean moduli of the first row are in agreement with François et al. [7], who used a method based on transformation groups to effect the projection. Note that the Euclidean projection for compliance again gives a different answer than for stiffness. Also, the bulk modulus for the log-Euclidean distance is the same as for the isotropic approximation, see Eq. (158).

This example illustrates the practical application of all three distance functions. The procedure is similar for materials in which the closest or best symmetry for approximation might be, for instance, transversely isotropic. The Euclidean projection follow from Eq. (68c) and the log-Euclidean moduli from Eqs. (152) and (153). The closest transversely isotropic tensor in the Riemannian norm can be found in a similar manner as above - in this case a system of four nonlinear equations for four unknowns is obtained, which can be solved by Newton's method.

\section{Conclusions}

Three distance functions for elastic tensors have been introduced and their application to elasticity examined. The Euclidean distance function is the most commonly used, but it lacks the property of invariance under inversion. Two distance functions with this important property have been described in detail: the log-Euclidean and the Riemannian norms. For each of the three distance functions we have developed a coordinate-independent procedure to find the closest elasticity tensor of a given symmetry to a set of moduli of a lower symmetry.

For the Euclidean distance function we have described a projection scheme using basis tensors, similar to vector space projections. Explicit forms for the projection operator have been given for isotropic, cubic, transversely isotropic, tetragonal and trigonal symmetries. The projection method was compared with the group transformation scheme and with the 21-dimensional approach. We also described the form of the projection operator using $6 \times 6$ matrices, which is easily implemented on a computer, and derived explicit expressions for the tensor complements/residues, and for the lengths of the projections. This allows one to decompose, in a Pythagorean sense, the elastic stiffness or compliance, although with different results for each.

Detailed and practical results have been presented for applying the logarithmic based distance functions to the same problem of approximating using a higher elastic symmetry. Both the log-Euclidean and the Riemannian distance functions are invariant under inversion. As such they offer an unambiguous method for determining the closest moduli of a given symmetry, providing unique results regardless of whether stiffness or compliance is considered. 
Calculation of the logarithmic distance functions requires evaluation of the logarithm, square root and exponential of tensors. Semi-analytical procedures for obtaining these for the most important, higher, elastic symmetries have been derived. For the first time, practical expressions are available for computing the logarithmic distances for symmetries higher than orthotropic, that is: isotropic, cubic, hexagonal (transversely isotropic), tetragonal, and trigonal. The numerical example considered illustrates how a data set with no symmetry can be reduced to find the optimally fitting cubic moduli. The Euclidean/Frobenius norm yields different results based on whether stiffness or compliances is use, but the log-Euclidean and the Riemannian distance functions provide unique answers.

\section{Appendix}

\section{A The Closest Tensors Using the Euclidean Norm}

Results for the Euclidean projection are summarized. We first give the explicit form of the projection for the various symmetries.

\section{A.1 Summary of the Euclidean Projections}

The projections onto the symmetry classes are expressed in terms of the elements of the six-dimensional second-order tensor $\widehat{\mathbf{C}}$ of Eq. (7). For the purpose of subsequent calculations, we take $\{\mathbf{a}, \mathbf{b}, \mathbf{c}\}=\left\{\mathbf{e}_{1}, \mathbf{e}_{2}, \mathbf{e}_{3}\right\}$. The symmetry classes are characterized by a distinct direction, the direction of the monoclinic symmetry plane, the direction perpendicular to the plane spanned by the normals to the planes of reflection symmetry for trigonal and tetragonal symmetry, the axis of transversely isotropic symmetry, a cube axis. In each case we take this direction as $\mathbf{e}_{3}$.

\section{A.1.1 Monoclinic and Orthorhombic Symmetry}

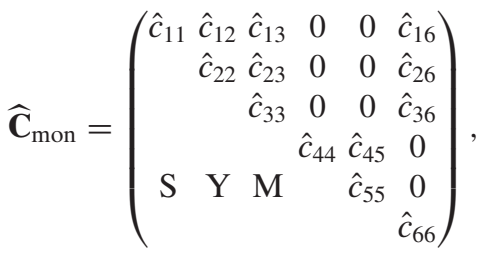

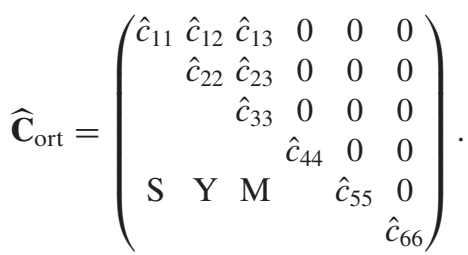




\section{A.1.2 Tetragonal Symmetry}

$\mathbb{C}_{\text {tet }}$ as defined in Eq. (37) becomes in $6 \times 6$ notation

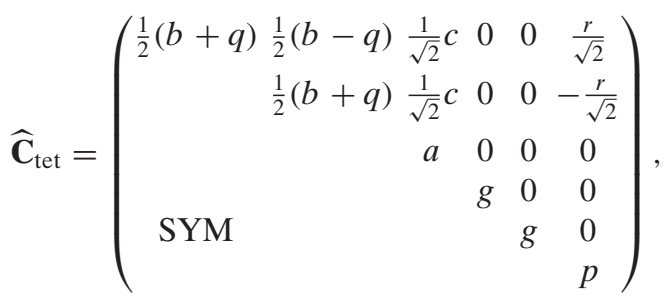

and the norm is

$$
\left\|\mathbb{C}_{\text {tet }}\right\|^{2}=a^{2}+b^{2}+2 c^{2}+p^{2}+q^{2}+2 r^{2}+2 g^{2} .
$$

Performing the inner products using Eq. (64), with $N=7$ and $\left\{\mathbb{V}_{1}, \mathbb{V}_{2}, \mathbb{V}_{3}, \mathbb{V}_{4}, \mathbb{V}_{5}\right.$, $\left.\mathbb{V}_{6}, \mathbb{V}_{6}\right\}=\left\{\mathbb{E}_{1}, \mathbb{E}_{2},\left(\mathbb{E}_{3}+\mathbb{E}_{4}\right), \mathbb{F}_{1}, \mathbb{F}_{2},\left(\mathbb{F}_{3}+\mathbb{F}_{4}\right), \mathbb{G}\right\}$ gives

$$
\begin{aligned}
& a=\hat{c}_{33}, \quad b=\frac{1}{2}\left(\hat{c}_{11}+\hat{c}_{22}+2 \hat{c}_{12}\right), \quad c=\frac{1}{\sqrt{2}}\left(\hat{c}_{13}+\hat{c}_{23}\right), \quad g=\frac{1}{2}\left(\hat{c}_{44}+\hat{c}_{55}\right), \\
& p=\hat{c}_{66}, \quad q=\frac{1}{2}\left(\hat{c}_{11}+\hat{c}_{22}-2 \hat{c}_{12}\right), \quad r=\frac{1}{\sqrt{2}}\left(\hat{c}_{16}-\hat{c}_{26}\right) .
\end{aligned}
$$

In summary, the projection is

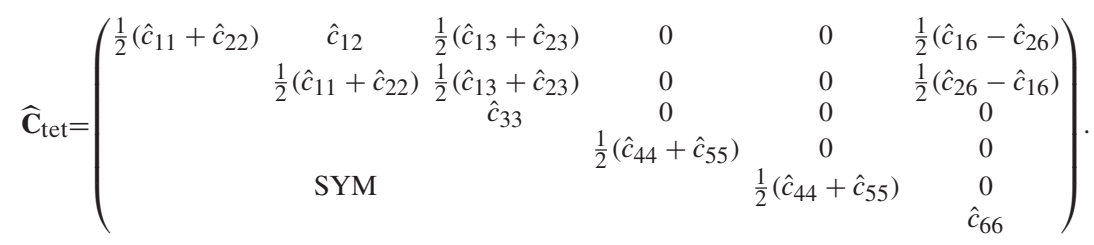

\section{A.1.3 Trigonal Symmetry}

$\mathbb{C}_{\text {trig }}$ as defined in Eq. (45) becomes

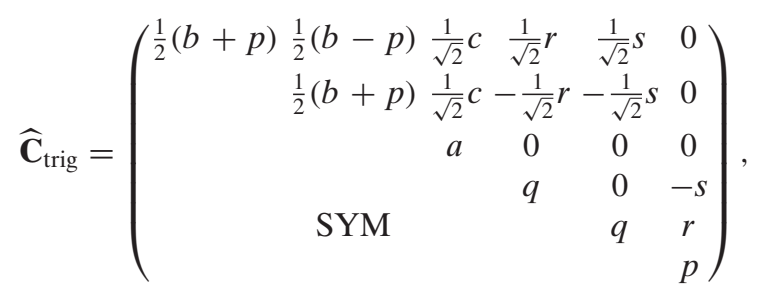

and the norm is

$$
\left\|\mathbb{C}_{\text {tet }}\right\|^{2}=a^{2}+b^{2}+2 c^{2}+2 p^{2}+2 q^{2}+4 r^{2}+4 s^{2},
$$


where

$$
\begin{aligned}
& a=\hat{c}_{33}, \quad b=\frac{1}{2}\left(\hat{c}_{11}+\hat{c}_{22}+2 \hat{c}_{12}\right), \quad c=\frac{1}{\sqrt{2}}\left(\hat{c}_{13}+\hat{c}_{23}\right), \quad p=\hat{c}_{66}, \\
& q=\frac{1}{2}\left(\hat{c}_{44}+\hat{c}_{55}\right), \quad r=\frac{1}{2 \sqrt{2}}\left(\hat{c}_{14}-\hat{c}_{24}\right)+\frac{1}{2} \hat{c}_{56}, \quad s=\frac{1}{2 \sqrt{2}}\left(\hat{c}_{15}-\hat{c}_{25}\right)-\frac{1}{2} \hat{c}_{46} .
\end{aligned}
$$

In this case,

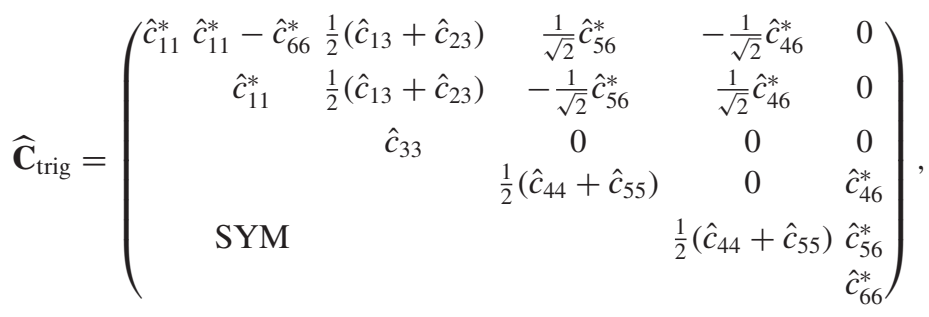

where

$$
\begin{aligned}
& \hat{c}_{11}^{*}=\frac{1}{8}\left(3 \hat{c}_{11}+3 \hat{c}_{22}+2 \hat{c}_{12}+2 \hat{c}_{66}\right), \\
& \hat{c}_{66}^{*}=\frac{1}{4}\left(\hat{c}_{11}+\hat{c}_{22}-2 \hat{c}_{12}+2 \hat{c}_{66}\right), \\
& \hat{c}_{46}^{*}=\frac{1}{2}\left(\hat{c}_{46}-\frac{1}{\sqrt{2}} \hat{c}_{15}+\frac{1}{\sqrt{2}} \hat{c}_{25}\right), \\
& \hat{c}_{56}^{*}=\frac{1}{2}\left(\hat{c}_{56}+\frac{1}{\sqrt{2}} \hat{c}_{14}-\frac{1}{\sqrt{2}} \hat{c}_{24}\right) .
\end{aligned}
$$

\section{A.1.4 Transverse Isotropy}

With $\mathbb{C}_{\text {hex }}$ defined as in Eq. (33), we have

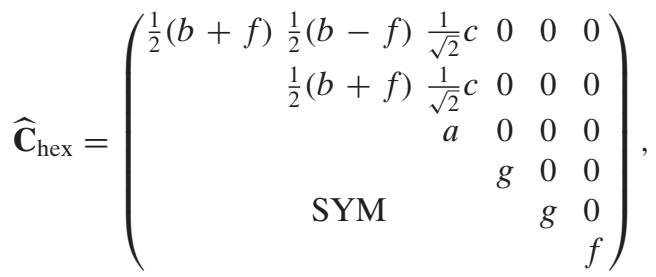

and

$$
\left\|\mathbb{C}_{\text {hex }}\right\|^{2}=a^{2}+b^{2}+2 c^{2}+2 f^{2}+2 g^{2} .
$$

The projection onto the basis tensors $\left\{\mathbb{V}_{1}, \mathbb{V}_{2}, \mathbb{V}_{3}, \mathbb{V}_{4}, \mathbb{V}_{5}\right\}=\left\{\mathbb{E}_{1}, \mathbb{E}_{2},\left(\mathbb{E}_{3}+\mathbb{E}_{4}\right)\right.$, $\mathbb{F}, \mathbb{G}\}$, yields $a, b, c$ and $g$ as given in Eq. (A.5) and

$$
f=\frac{1}{2}(p+q)=\frac{1}{4}\left(\hat{c}_{11}+\hat{c}_{22}+2 \hat{c}_{66}-2 \hat{c}_{12}\right) .
$$


Thus,

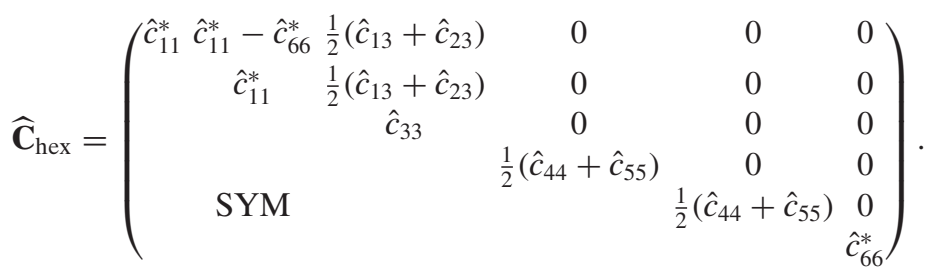

\section{A.1.5 Cubic Symmetry}

The 6-D matrix associated with $\mathbb{C}_{\text {cub }}$ defined in Eq. (28) is

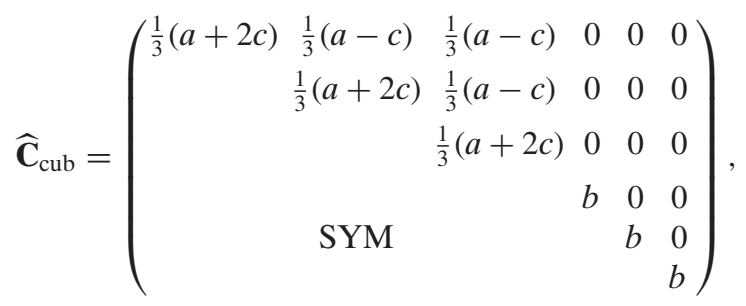

with length

$$
\left\|\mathbb{C}_{\text {cub }}\right\|^{2}=a^{2}+3 b^{2}+2 c^{2} .
$$

The Euclidean projection onto cubic symmetry follows from Eq. (64) with $N=3$ and $\left\{\mathbb{V}_{1}, \mathbb{V}_{2}, \mathbb{V}_{3}\right\}=\{\mathbb{J}, \mathbb{L}, \mathbb{M}\}$, and gives

$$
\begin{aligned}
& a=\frac{1}{3}\left(\hat{c}_{11}+\hat{c}_{22}+\hat{c}_{33}+2 \hat{c}_{12}+2 \hat{c}_{13}+2 \hat{c}_{23}\right) \\
& b=\frac{1}{3}\left(\hat{c}_{44}+\hat{c}_{55}+\hat{c}_{66}\right), \\
& c=\frac{1}{3}\left(\hat{c}_{11}+\hat{c}_{22}+\hat{c}_{33}-\hat{c}_{12}-\hat{c}_{23}-\hat{c}_{31}\right) .
\end{aligned}
$$

Note that $a=3 \kappa$ where $\kappa$ defined in Eq. $(23)_{1}$. In summary, the projection is

$$
\widehat{\mathbf{C}}_{\mathrm{cub}}=\left(\begin{array}{cccccc}
\hat{c}_{11}^{C} & \hat{c}_{12}^{C} & \hat{c}_{12}^{C} & 0 & 0 & 0 \\
& \hat{c}_{11}^{C} & \hat{c}_{12}^{C} & 0 & 0 & 0 \\
& & \hat{c}_{11}^{C} & 0 & 0 & 0 \\
& & & \hat{c}_{66}^{C} & 0 & 0 \\
& \mathrm{SYM} & & & \hat{c}_{66}^{C} & 0 \\
& & & & & \hat{c}_{66}^{C}
\end{array}\right)
$$

where

$$
\hat{c}_{11}^{C}=\frac{1}{3}\left(\hat{c}_{11}+\hat{c}_{22}+\hat{c}_{33}\right), \quad \hat{c}_{12}^{C}=\frac{1}{3}\left(\hat{c}_{12}+\hat{c}_{13}+\hat{c}_{23}\right), \quad \hat{c}_{66}^{C}=\frac{1}{3}\left(\hat{c}_{44}+\hat{c}_{55}+\hat{c}_{66}\right) .
$$




\section{A.1.6 Isotropy}

With $\mathbb{C}_{\text {iso }}$ as given by Eq. (22), we have

$$
\widehat{\mathbf{C}}_{\text {iso }}=\left(\begin{array}{rrrr}
\kappa+\frac{4}{3} \mu \kappa-\frac{2}{3} \mu \kappa-\frac{2}{3} \mu & 0 & 0 & 0 \\
\kappa+\frac{4}{3} \mu \kappa-\frac{2}{3} \mu & 0 & 0 & 0 \\
\kappa+\frac{4}{3} \mu & 0 & 0 & 0 \\
2 \mu & 0 & 0 \\
\text { SYM } & & 2 \mu & 0 \\
& & & 2 \mu
\end{array}\right),
$$

where $\kappa$ and $\mu$ are defined in Eq. (23). These follow from the general projection formula (64) with $N=2$ and $\left\{\mathbb{V}_{1}, \mathbb{V}_{2}\right\}=\{\mathbb{J}, \mathbb{K}\}$.

\section{A.2 The Space Between Isotropy and Transverse Isotropy}

We use a Gram-Schmid approach to define an orthogonal subspace between isotropy and transverse isotropy (hexagonal symmetry). Noting that

$$
\mathbb{J}=\frac{1}{3}\left[\mathbb{E}_{1}+2 \mathbb{E}_{2}+\sqrt{2}\left(\mathbb{E}_{3}+\mathbb{E}_{4}\right)\right], \quad \mathbb{K}=\frac{1}{3}\left[2 \mathbb{E}_{1}+\mathbb{E}_{2}-\sqrt{2}\left(\mathbb{E}_{3}+\mathbb{E}_{4}\right)+3 \mathbb{F}+3 \mathbb{G}\right],
$$

we introduce

$$
\begin{aligned}
& \mathbb{L}_{1}=\mathbb{G}-\mathbb{F}, \\
& \mathbb{L}_{2}=2 \mathbb{E}_{1}-2 \mathbb{E}_{2}+\frac{1}{\sqrt{2}}\left(\mathbb{E}_{3}+\mathbb{E}_{4}\right), \\
& \mathbb{L}_{3}=8 \mathbb{E}_{1}+4 \mathbb{E}_{2}-4 \sqrt{2}\left(\mathbb{E}_{3}+\mathbb{E}_{4}\right)-3 \mathbb{F}-3 \mathbb{G} .
\end{aligned}
$$

Thus, $\left\langle\mathbb{L}_{i}, \mathbb{L}_{j}\right\rangle=0, i \neq j$, and $\left\langle\mathbb{L}_{i}, \mathbb{J}\right\rangle=0,\left\langle\mathbb{L}_{i}, \mathbb{K}\right\rangle=0, i=1,2,3$. Hence $\left\{\mathbb{V}_{i}, i=1\right.$, $2, \ldots 5\}=\left\{\mathbb{J}, \mathbb{K}, \mathbb{L}_{1}, \mathbb{L}_{2}, \mathbb{L}_{3}\right\}$ form a basis for Hex, with $\mathbf{D}=\operatorname{diag}(1,5,4,9,180)$. Eq. (67) gives

$$
\begin{aligned}
P_{\text {hex }} & =\mathbb{J} \otimes \mathbb{J}+\frac{1}{5} \mathbb{K} \otimes \mathbb{K}+\frac{1}{4} \mathbb{L}_{1} \otimes \mathbb{L}_{1}+\frac{1}{9} \mathbb{L}_{2} \otimes \mathbb{L}_{2}+\frac{1}{180} \mathbb{L}_{3} \otimes \mathbb{L}_{3} \\
& =P_{\text {iso }}+P_{\text {hex } / \text { iso }} .
\end{aligned}
$$

Thus, we have the explicit decomposition into orthogonal complements $\mathbb{C}_{\mathrm{hex}}=\mathbb{C}_{\mathrm{iso}}+$ $\mathbb{C}_{\text {hex/iso, }}$, where

$$
\mathbb{C}_{\text {hex } / \text { iso }}=d_{1} \mathbb{L}_{1}+d_{2} \mathbb{L}_{2}+d_{3} \mathbb{L}_{3}, \quad\left\|\mathbb{C}_{\text {hex } / \text { iso }}\right\|^{2}=4 d_{1}^{2}+9 d_{2}^{2}+180 d_{3}^{2},
$$

with

$$
d_{1}=\frac{1}{2}(g-f), \quad d_{2}=\frac{1}{9}(2 a-2 b+\sqrt{2} c), \quad d_{3}=\frac{1}{90}(4 a+2 b-4 \sqrt{2} c-3 f-3 g),
$$


and $a, \ldots, g$ are given by A.5 and A.14. Eliminating the latter gives

$$
\begin{aligned}
& d_{1}=\frac{1}{8}\left[2\left(\hat{c}_{44}+\hat{c}_{55}+\hat{c}_{12}\right)-\left(\hat{c}_{11}+\hat{c}_{22}+2 \hat{c}_{66}\right)\right], \\
& d_{2}=\frac{1}{9}\left[\hat{c}_{13}+\hat{c}_{23}+2 \hat{c}_{33}-\left(\hat{c}_{11}+\hat{c}_{22}+2 \hat{c}_{12}\right)\right], \\
& d_{3}=\frac{1}{360}\left[\hat{c}_{11}+\hat{c}_{22}+14 \hat{c}_{12}+16\left(\hat{c}_{33}-\hat{c}_{13}-\hat{c}_{23}\right)-6\left(\hat{c}_{44}+\hat{c}_{55}+\hat{c}_{66}\right)\right] .
\end{aligned}
$$

\section{A.3 Complements}

Some orthogonal complements or residues [3] between the symmetry classes are presented here. These lead to the explicit expressions for elastic lengths in the next subsection, Eqs. (A.30) and (A.31). Thus,

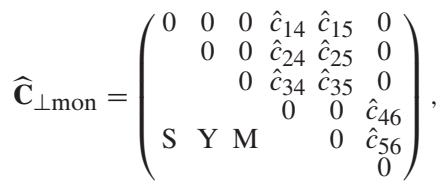

$$
\begin{aligned}
& \widehat{\mathbf{C}}_{\text {mon/ort }}=\left(\begin{array}{cccccc}
0 & 0 & 0 & 0 & 0 & \hat{c}_{16} \\
0 & 0 & 0 & 0 & \hat{c}_{26} \\
& 0 & 0 & 0 & \hat{c}_{36} \\
& & 0 & \hat{c}_{45} & 0 \\
\mathrm{~S} & \mathrm{Y} & \mathrm{M} & & 0 & 0 \\
& & & & & 0
\end{array}\right),
\end{aligned}
$$

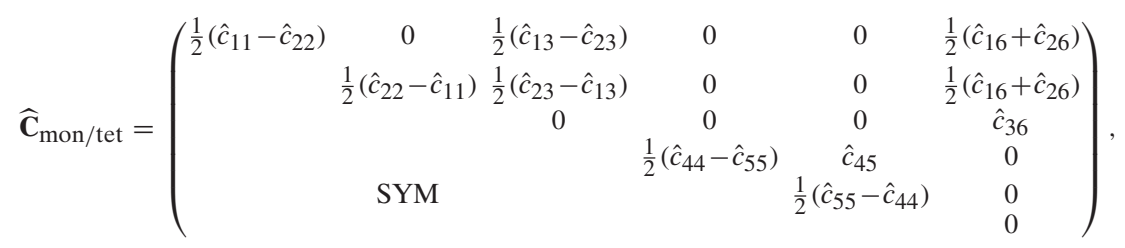

$$
\begin{aligned}
& \widehat{\mathbf{C}}_{\text {ort } / \mathrm{hex}}=\left(\begin{array}{cccccc}
r_{0}+\frac{1}{2}\left(\hat{c}_{11}-\hat{c}_{22}\right) & -r_{0} & \frac{1}{2}\left(\hat{c}_{13}-\hat{c}_{23}\right) & 0 & 0 & 0 \\
& r_{0}+\frac{1}{2}\left(\hat{c}_{22}-\hat{c}_{11}\right) & \frac{1}{2}\left(\hat{c}_{23}-\hat{c}_{13}\right) & 0 & 0 & 0 \\
& & 0 & 0 & 0 & 0 \\
& & & \frac{1}{2}\left(\hat{c}_{44}-\hat{c}_{55}\right) & 0 & 0 \\
& & & & \frac{1}{2}\left(\hat{c}_{55}-\hat{c}_{44}\right) & 0 \\
& & & & & -2 r_{0}
\end{array}\right),(\mathrm{A} .28 \mathrm{~d})
\end{aligned}
$$




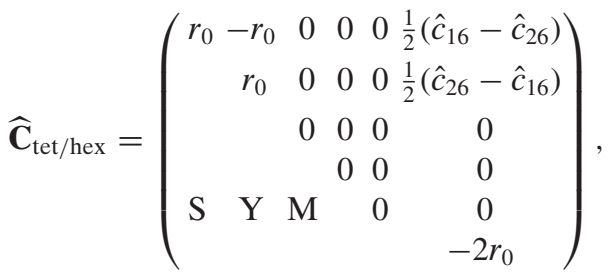

$$
\begin{aligned}
& \widehat{\mathbf{C}}_{\text {tet } / \mathrm{cub}}=\left(\begin{array}{cccccc}
r_{1}-2 r_{2} & r_{2} & 0 & 0 & \frac{1}{2}\left(\hat{c}_{16}-\hat{c}_{26}\right) \\
& r_{1} & r_{2} & 0 & 0 & \frac{1}{2}\left(\hat{c}_{26}-\hat{c}_{16}\right) \\
& & -2 r_{1} & 0 & 0 & 0 \\
& & & r_{3} & 0 & 0 \\
\mathrm{~S} & \mathrm{Y} & \mathrm{M} & & r_{3} & 0 \\
& & & & & -2 r_{3}
\end{array}\right),
\end{aligned}
$$

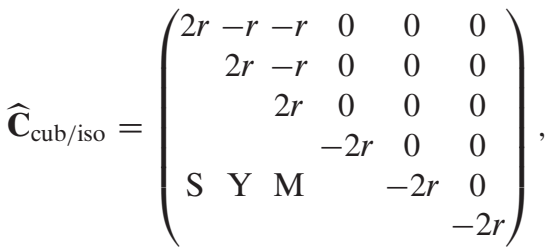

where

$$
\begin{array}{lrl}
r_{0}=\frac{1}{8}\left(\hat{c}_{11}+\hat{c}_{22}-2 \hat{c}_{12}-2 \hat{c}_{66}\right), & r_{1}=\frac{1}{6}\left(\hat{c}_{11}+\hat{c}_{22}-2 \hat{c}_{33}\right), \\
r_{2}=\frac{1}{6}\left(\hat{c}_{13}+\hat{c}_{23}-2 \hat{c}_{12}\right), & r_{3}=\frac{1}{6}\left(\hat{c}_{44}+\hat{c}_{55}-2 \hat{c}_{66}\right), \\
r=\frac{1}{15}\left(\hat{c}_{11}+\hat{c}_{22}+\hat{c}_{33}-\hat{c}_{12}-\hat{c}_{13}-\hat{c}_{23}-\hat{c}_{44}-\hat{c}_{55}-\hat{c}_{66}\right) .
\end{array}
$$

Also, $\widehat{\mathbf{C}}_{\text {hex/iso }}$ follows from Eq. (A.27) and (A.25) but is not given explicitly because of its length, and neither are the complements involving $\widehat{\mathbf{C}}_{\text {trig }}$.

A.4 The Euclidean Distances

Three projections of the elastic length are as follows

$$
\begin{aligned}
\|\mathbb{C}\|^{2} & =\left\|\mathbb{C}_{\text {iso }}\right\|^{2}+\left\|\mathbb{C}_{\text {cub } / \text { iso }}\right\|^{2}+\left\|\mathbb{C}_{\text {tet } / \text { cub }}\right\|^{2}+\left\|\mathbb{C}_{\text {mon } / \text { tet }}\right\|^{2}+\left\|\mathbb{C}_{\perp \text { mon }}\right\|^{2} \\
& =\left\|\mathbb{C}_{\text {iso }}\right\|^{2}+\left\|\mathbb{C}_{\text {hex } / \text { iso }}\right\|^{2}+\left\|\mathbb{C}_{\text {tet } / \text { hex }}\right\|^{2}+\left\|\mathbb{C}_{\text {mon/tet }}\right\|^{2}+\left\|\mathbb{C}_{\perp \text { mon }}\right\|^{2} \\
& =\left\|\mathbb{C}_{\text {iso }}\right\|^{2}+\left\|\mathbb{C}_{\text {hex/iso }}\right\|^{2}+\left\|\mathbb{C}_{\text {ort } / \text { hex }}\right\|^{2}+\left\|\mathbb{C}_{\text {mon/ort }}\right\|^{2}+\left\|\mathbb{C}_{\perp \text { mon }}\right\|^{2} .
\end{aligned}
$$


These can be explicitly calculated using

$$
\begin{aligned}
\left\|\widehat{\mathbf{C}}_{\perp \text { mon }}\right\|^{2}= & 2\left(\hat{c}_{14}^{2}+\hat{c}_{15}^{2}+\hat{c}_{24}^{2}+\hat{c}_{25}^{2}+\hat{c}_{34}^{2}+\hat{c}_{35}^{2}+\hat{c}_{46}^{2}+\hat{c}_{56}^{2}\right), \\
\left\|\widehat{\mathbf{C}}_{\text {mon } / \text { ort }}\right\|^{2}= & 2\left(\hat{c}_{16}^{2}+\hat{c}_{26}^{2}+\hat{c}_{36}^{2}+\hat{c}_{45}^{2}\right), \\
\left\|\widehat{\mathbf{C}}_{\text {ort } / \text { hex }}\right\|^{2}= & \frac{1}{8}\left(\hat{c}_{11}+\hat{c}_{22}-2 \hat{c}_{12}-2 \hat{c}_{66}\right)^{2}+\frac{1}{2}\left(\hat{c}_{11}-\hat{c}_{22}\right)^{2}+\left(\hat{c}_{13}-\hat{c}_{23}\right)^{2}+\frac{1}{2}\left(\hat{c}_{44}-\hat{c}_{55}\right)^{2}, \\
\left\|\widehat{\mathbf{C}}_{\text {mon } / \text { tet }}\right\|^{2}= & \frac{1}{2}\left(\hat{c}_{11}-\hat{c}_{22}\right)^{2}+\left(\hat{c}_{13}-\hat{c}_{23}\right)^{2}+\frac{1}{2}\left(\hat{c}_{44}-\hat{c}_{55}\right)^{2}+\left(\hat{c}_{16}+\hat{c}_{26}\right)^{2}+2 \hat{c}_{45}^{2}+2 \hat{c}_{36}^{2}, \\
\left\|\widehat{\mathbf{C}}_{\text {tet } / \text { cub }}\right\|^{2}= & \frac{1}{6}\left(\hat{c}_{11}+\hat{c}_{22}-2 \hat{c}_{33}\right)^{2}+\frac{1}{3}\left(\hat{c}_{13}+\hat{c}_{23}-2 \hat{c}_{12}\right)^{2} \\
& +\frac{1}{6}\left(\hat{c}_{44}+\hat{c}_{55}-2 \hat{c}_{66}\right)^{2}+\left(\hat{c}_{16}-\hat{c}_{26}\right)^{2}, \\
\left\|\widehat{\mathbf{C}}_{\text {cub } / \text { iso }}\right\|^{2}= & \frac{2}{15}\left(\hat{c}_{11}+\hat{c}_{22}+\hat{c}_{33}-\hat{c}_{12}-\hat{c}_{13}-\hat{c}_{23}-\hat{c}_{44}-\hat{c}_{55}-\hat{c}_{66}\right)^{2}, \\
\left\|\widehat{\mathbf{C}}_{\text {tet } / \text { hex }}\right\|^{2}= & \frac{1}{8}\left(\hat{c}_{11}+\hat{c}_{22}-2 \hat{c}_{12}-2 \hat{c}_{66}\right)^{2}+\left(\hat{c}_{16}-\hat{c}_{26}\right)^{2} \\
\left\|\widehat{\mathbf{C}}_{\text {hex } / \text { iso }}\right\|^{2}= & \frac{1}{16}\left[2\left(\hat{c}_{44}+\hat{c}_{55}+\hat{c}_{12}\right)-\left(\hat{c}_{11}+\hat{c}_{22}+2 \hat{c}_{66}\right)\right]^{2} \\
& +\frac{1}{9}\left[\hat{c}_{13}+\hat{c}_{23}+2 \hat{c}_{33}-\left(\hat{c}_{11}+\hat{c}_{22}+2 \hat{c}_{12}\right)\right]^{2} \\
& +\frac{1}{720}\left[\hat{c}_{11}+\hat{c}_{22}+14 \hat{c}_{12}+16\left(\hat{c}_{33}-\hat{c}_{13}-\hat{c}_{23}\right)-6\left(\hat{c}_{44}+\hat{c}_{55}+\hat{c}_{66}\right)\right]^{2},
\end{aligned}
$$

and Eqs. (23) and (58) give

$$
\begin{aligned}
\left\|\mathbb{C}_{\text {iso }}\right\|^{2}= & \frac{1}{9}\left(\hat{c}_{11}+\hat{c}_{22}+\hat{c}_{33}+2 \hat{c}_{12}+2 \hat{c}_{13}+2 \hat{c}_{23}\right)^{2} \\
& +\frac{1}{45}\left[2\left(\hat{c}_{11}+\hat{c}_{22}+\hat{c}_{33}-\hat{c}_{12}-\hat{c}_{23}-\hat{c}_{31}\right)+3\left(\hat{c}_{44}+\hat{c}_{55}+\hat{c}_{66}\right)\right]^{2} .
\end{aligned}
$$

All versions of the elastic length in Eq. (A.30) are the sum of 21 positive numbers, the same as the raw form of the squared length in any rectangular coordinate system using the Voigt elements. However, the three sets of 21 positive numbers in Eq. (A.30) contain far more information about the underlying elastic symmetry of the material.

\section{B Exponential, Logarithm and Square Root of Hermitian Matrices}

Let $A$ be the $2 \times 2$ Hermitian matrix

$$
A=\left[\begin{array}{cc}
a & c+i d \\
c-i d & b
\end{array}\right]
$$

where $a, b, c$ and $d$ are real numbers. The matrix can be decomposed into the sum of an isotropic part and a deviatoric part,

$$
A=A_{\text {iso }}+A_{\text {dev }}=\left[\begin{array}{ll}
\alpha & 0 \\
0 & \alpha
\end{array}\right]+\left[\begin{array}{cc}
\beta & c+i d \\
c-i d & -\beta
\end{array}\right],
$$


where $\alpha=(a+b) / 2, \beta=(a-b) / 2$ and $\gamma=\sqrt{\beta^{2}+c^{2}+d^{2}}$. Note that $A_{\mathrm{dev}}$ vanishes if and only if $\gamma=0$, i.e., $a=b$ and $c=d=0$, and that $A_{\mathrm{dev}}^{2}=\gamma^{2} I$. The eigenvalues of $A$ are real and given by

$$
\lambda_{ \pm}=\alpha \pm \gamma
$$

For convenience we introduce the matrix

$$
A^{\prime}= \begin{cases}I & \text { if } \quad \gamma=0 \\ \frac{1}{\gamma} A_{\mathrm{dev}} & \text { if } \quad \gamma>0\end{cases}
$$

so that $A^{\prime 2}=I$. The exponential of $A$ is then

$$
\exp A=e^{\alpha}\left(\cosh \gamma I+\sinh A^{\prime}\right)= \begin{cases}e^{\alpha} I & \text { if } \gamma=0, \\ e^{\alpha}\left(\cosh \gamma I+\frac{\sinh \gamma}{\gamma} A_{\mathrm{dev}}\right) & \text { if } \quad \gamma>0 .\end{cases}
$$

When $a b-c^{2} \neq 0$ the matrix $A$ is invertible and its inverse is

$$
\begin{aligned}
A^{-1} & =\frac{1}{a b-c^{2}-d^{2}}\left[\begin{array}{cc}
b & -(c+i d) \\
-(c-i d) & a
\end{array}\right]=\frac{1}{a b-c^{2}-d^{2}}\left(\alpha I-\gamma A^{\prime}\right) \\
& =\frac{1}{a b-c^{2}-d^{2}}\left(\alpha I-A_{\mathrm{dev}}\right) .
\end{aligned}
$$

If $a>0, b>0$ and $a b-c^{2}-d^{2}>0$, then $A$ is positive definite. Let $\delta=$ $\sqrt{a b-c^{2}-d^{2}}$, then the positive-definite square root of $A$ is

$$
A^{1 / 2}=\frac{1}{\sqrt{2(\alpha+\delta)}}\left[\begin{array}{l}
a+\delta c+i d \\
c-i d b+\delta
\end{array}\right]=\frac{1}{\sqrt{2(\alpha+\delta)}}\left[(\alpha+\delta) I+A_{\mathrm{dev}}\right],
$$

and the inverse of its square root is given by

$$
A^{-1 / 2}=\frac{1}{\delta \sqrt{2(\alpha+\delta)}}\left[\begin{array}{cc}
b+\delta & -(c+i d) \\
-(c-i d) & a+\delta
\end{array}\right]=\frac{1}{\delta \sqrt{2(\alpha+\delta)}}\left[(\alpha+\delta) I-A_{\mathrm{dev}}\right] .
$$

The logarithm is

$$
\log A=\ln \delta I+\ln \sqrt{\frac{\alpha+\gamma}{\alpha-\gamma}} A^{\prime}= \begin{cases}\ln \delta I & \text { if } \gamma=0, \\ \ln \delta I+\frac{1}{\gamma} \ln \sqrt{\frac{\alpha+\gamma}{\alpha-\gamma}} A_{\mathrm{dev}} & \text { if } \gamma>0 .\end{cases}
$$

More generally, for any analytic function $f$ we have

$$
f(A)= \begin{cases}f(\alpha) I & \text { if } \gamma=0 \\ \frac{f(\alpha+\gamma)+f(\alpha-\gamma)}{2} I+\frac{f(\alpha+\gamma)-f(\alpha-\gamma)}{2 \gamma} A_{\mathrm{dev}} & \text { if } \gamma>0 .\end{cases}
$$

The Cayley-Hamilton theorem states that

$$
\operatorname{det}(A) I-\operatorname{tr}(A) A+A^{2}=0 .
$$

Therefore, any analytic function of $A$ can be written as a linear combination of $I, A$ and $A^{2}$, or, if $A$ is invertible, as a linear combination of $I, A$ and $A^{-1}$. Using the fact 
that $2 A_{\mathrm{dev}}=A-\delta^{2} A^{-1}$, we have the alternative expressions for the exponential, logarithm and square roots

$$
\begin{aligned}
\exp A & =e^{\alpha}\left[\cosh \gamma I+\frac{\sinh \gamma}{2 \gamma}\left(A-\delta^{2} A^{-1}\right)\right], \\
\log A & =\ln \delta I+\frac{1}{4 \gamma} \ln \frac{\alpha+\gamma}{\alpha-\gamma}\left(A-\delta^{2} A^{-1}\right), \\
A^{1 / 2} & =\frac{1}{\sqrt{2(\alpha+\delta)}}[\delta I+A], \\
A^{-1 / 2} & =\frac{1}{\sqrt{2(\alpha+\delta)}}\left[I+\delta A^{-1}\right] .
\end{aligned}
$$

We recall that for any $n \times n$ matrix $M$ we have

$$
\operatorname{det}(\exp M)=e^{\operatorname{tr} M} .
$$

Therefore, when $M=\log P$ for some matrix $P$ we get

$$
\operatorname{det} P=e^{\operatorname{tr}(\log P)} .
$$

\section{References}

1. Moakher, M.: On the averaging of symmetric positive-definite tensors. J. Elast. 82, 273-296 (2006)

2. Arsigny, V., Fillard, P., Pennec, X., Ayache, N.: Fast and simple calculus on tensors in the LogEuclidean framework. In: Duncan, J., Gerig, G. (eds.) Proc. 8th International Conference on Medical Image Computing and Computer-Assisted Intervention - MICCAI 2005, Part I, vol. 3749 of LNCS, Palm Springs, California, pp. 115-122. Springer, Berlin Heidelberg New York (2005)

3. Gazis, D.C., Tadjbakhsh, I., Toupin, R.A.: The elastic tensor of given symmetry nearest to an anisotropic elastic tensor. Acta Crystallogr. 16, 917-922 (1963)

4. Fedorov, F.I.: Theory of Elastic Waves in Crystals. Plenum, New York (1968)

5. Norris, A.N.: Elastic moduli approximation of higher symmetry for the acoustical properties of an anisotropic material. J. Acoust. Soc. Am. 119, 2114-2121 (2006)

6. Forte, S., Vianello, M.: Symmetry classes for elasticity tensors. J. Elast. 43, 81-108 (1996)

7. François, M., Geymonat, G., Berthaud, Y.: Determination of the symmetries of an experimentally determined stiffness tensor, application to acoustic measurements. Int. J. Solids Struct. 35(31-32), 4091-4106 (1998)

8. Arts, R.J., Helbig, K., Rasolofosaon, P.N.J.: General anisotropic elastic tensors in rocks: approximation, invariants and particular directions. In: Expanded Abstracts, 61st Annual International Meeting, Society of Exploration Geophysicists, ST2.4, pp. 1534-1537. Society of Exploration Geophysicists, Tulsa, Oklahoma (1991)

9. Helbig, K.: Representation and approximation of elastic tensors. In: Fjaer, E., Holt, R.M., Rathore, J.S. (eds.) Seismic Anisotropy, pp. 37-75. Society of Exploration Geophysicists, Tulsa, Oklahoma (1996)

10. Cavallini, F.: The best isotropic approximation of an anisotropic Hooke's law. Boll. Geofis. Teor. Appl. 40, 1-18 (1999)

11. Gangi, A.F.: Fourth-order elastic-moduli tensors by inspection. In: Ikelle, L., Gangi, A.F. (eds.) Anisotropy 2000: Fractures, Converted Waves and Case Studies. Proc. 9th International Workshop on Seismic Anisotropy (9IWSA), pp. 1-10. Society of Exploration Geophysicists, Tulsa, Oklahoma (2000)

12. Rychlewski, J.: On Hooke’s law. Prikl. Mat. Meh. 48, 303-314 (1984)

13. Cowin, S.C., Mehrabadi, M.M.: The structure of the linear anisotropic elastic symmetries. J. Mech. Phys. Solids 40, 1459-1471 (1992) 
14. Browaeys, J.T., Chevrot, S.: Decomposition of the elastic tensor and geophysical applications. Geophys. J. Int. 159, 667-678 (2004)

15. Dellinger, J.: Computing the optimal transversely isotropic approximation of a general elastic tensor. Geophysics 70, I1-I10 (2005)

16. Dellinger, J., Vasicek, D., Sondergeld, C.: Kelvin notation for stabilizing elastic-constant inversion. Rev. Inst. Fr. Pét. 53, 709-719 (1998)

17. Norris, A.N.: The isotropic material closest to a given anisotropic material. J. Mech. Materials Struct. 1, 223-238 (2006)

18. Chadwick, P., Vianello, M., Cowin, S.C.: A new proof that the number of linear elastic symmetries is eight. J. Mech. Phys. Solids 49, 2471-2492 (2001)

19. Walpole, L.J.: Fourth rank tensors of the thirty-two crystal classes: multiplication tables. Proc. R. Soc. A A391, 149-179 (1984)

20. Mehrabadi, M.M., Cowin, S.C.: Eigentensors of linear anisotropic elastic materials. Q. J. Mech. Appl. Math. 43, 15-41 (1990)

21. Musgrave, M.J.P.: Crystal Acoustics. Acoustical Society of America, New York (2003)

22. Lang, S.: Fundamentals of Differential Geometry. Springer, Berlin Heidelberg New York (1998)

23. Bhatia, R.: Matrix Analysis. Springer, Berlin Heidelberg New York (1997)

24. Moakher, M.: A differential geometric approach to the geometric mean of symmetric positivedefinite matrices. SIAM J. Matrix Anal. Appl. 26, 735-747 (2005)

25. Thomson, W.: Elements of a mathematical theory of elasticity. Philos. Trans. R. Soc. Lond. 146, 481-498 (1856)

26. Sutcliffe, J.: Spectral decomposition of the elasticity tensor. J. Appl. Mech. ASME 59, 762-773 (1993)

27. Theocaris, P.S., Sokolis, D.P.: Spectral decomposition of the compliance fourth-rank tensor for orthotropic materials. Arch. Appl. Mech. 70, 289-306 (2000)

28. Kunin, I.A.: Elastic Media with Microstructure. Springer, Berlin Heidelberg New York (1982)

29. Surrel, Y.: A new description of the tensors of elasticity based upon irreducible representations. Eur. J. Mech. A, Solids 12, 219-235 (1993)

30. Forte, S., Vianello, M.: Functional bases for transversely isotropic and transversely hemitropic invariants of elasticity tensors. Q. J. Mech. Appl. Math. 51, 543-552 (1998)

31. Xiao, H.: Invariant characteristic representations for classical and micropolar anisotropic elasticity tensors. J. Elast. 40, 239-265 (1995)

32. Xiao, H.: On symmetries and anisotropies of classical and micropolar linear elasticities: a new method based upon a complex vector basis and some systematic results. J. Elast. 49, 129-162 (1998)

33. Bóna, A., Bucataru, I., Slawinski, A.: Characterization of elasticity-tensor symmetries using SU(2). J. Elast. 75, 267-289 (2004)

34. Bóna, A., Bucataru, I., Slawinski, A.: Material symmetries of elasticity tensors. Q. J. Mech. Appl. Math. 57, 583-598 (2004)

35. Cowin, S.C., Mehrabadi, M.M.: Anisotropic symmetries of linear elasticity. Appl. Mech. Rev. 48, 247-285 (1995)

36. Backus, G.: A geometrical picture of anisotropic elastic tensors. Rev. Geophys. Space Phys. 8, 633-671 (1970)

37. Cowin, S.C.: Properties of the anisotropic elasticity tensor. Q. J. Mech. Appl. Math. 42, 249-266 (1989)

38. Baerheim, R.: Harmonic decomposition of the anisotropic elasticity tensor. Q. J. Mech. Appl. Math. 46, 391-418 (1993)

39. Zou, W.N., Zheng, Q.S.: Maxwell's multipole representation of traceless symmetric tensors and its application to functions of high-order tensors. Proc. R. Soc. A A459, 527-538 (2003)

40. Baerheim, R., Helbig, K.: Decomposition of the anisotropic elastic tensor in base tensors. Can. J. Explor. Geophys. 29, 41-50 (1993)

41. Smith, G.F., Rivlin, R.S.: Stress deformation relations for anisotropic solids. Arch. Ration. Mech. Anal. 1, 107-112 (1957)

42. Tu, Y.-O.: The decomposition of an anisotropic elastic tensor. Acta Crystallogr. A24, 273-282 (1968)

43. Spencer, A.J.M.: Theory of invariants. In: Eringen, A.C. (ed) Continuum Physics, vol. 1. Academic, New York (1971)

44. Betten, J.: Integrity basis for a second-order and a fourth-order tensor. Int. J. Math. Math. Sci. 5, 87-96 (1981) 
45. Xiao, H.: On anisotropic invariants of a symmetric tensor: crystal classes, quasi-crystalclasses and others. Proc. R. Soc. A 454, 1217-1240 (1998)

46. Elata, D., Rubin, M.B.: A new representation for the strain energy of anisotropic elastic materials with application to damage evolution in brittle materials. Mech. Mater. 19, 171-192 (1995)

47. François, M., Berthaud, Y., Geymonat, G.: Une nouvelle analyse des symétries d'un matériau élastique anisotrope. exemple d'utilisation à partir de mesures ultrasonores. C. R. Acad. Sci. Paris, II 322, 87-94 (1996)

48. Cowin, S.C., Mehrabadi, M.M.: On the identification of material symmetry for anisotropic elastic materials. Q. J. Mech. Appl. Math. 40, 451-476 (1987) 